\title{
Synthesis of Thiourea-Oxazolines, a New Class of Chiral S,N-Heterobidentate Ligands: Application in Pd-catalyzed Asymmetric Bis-methoxycarbonylation of Terminal Olefins
}

Bo Liang, ${ }^{\dagger}$ Jing Liu, ${ }^{\dagger}$ Ying-Xiang Gao, ${ }^{\dagger}$ Kittiya, Wongkhan, ${ }^{\ddagger}$ Dong-Xu Shu, ${ }^{\dagger}$ Yu Lan,${ }^{\dagger}$ Ang Li, ${ }^{\dagger}$ Andrei S. Batsanov, ${ }^{\ddagger}$ Judith A. K. Howard, ${ }^{\star}$ Todd B. Marder, ${ }^{\ddagger}$ Jia-Hua Chen, ${ }^{* \dagger}$ and Zhen Yang ${ }^{* \dagger}$

\begin{abstract}
${ }^{a}$ Key Laboratory of Bioorganic Chemistry and Molecular Engineering of Ministry of Education and Beijing National Laboratory for Molecular Science (BNLMS), College of Chemistry, the State Key Laboratory of Natural and Biomimetic Drugs, School of Pharmaceutical Science, and Laboratory of Chemical Genomics, Shenzhen Graduate School, Peking University, Beijing, 100871, China;
\end{abstract}

${ }^{b}$ Department of Chemistry, Durham University, Durham, DH1 3LE, UK

zyang@pku.edu.cn

\section{Supporting Information}

\section{Table of Contents}

Page S3-S6: General Information, synthetic procedure and spectroscopic data for the syntheses of compounds $9,10,11,12,13,14,15$, and 17

Page S7-S18: General procedure for the Pd-catalyzed asymmetric bis-methoxycarbonylations of terminal olefins and spectroscopic data for the syntheses of compounds $2, \mathbf{1 8 b}, \mathbf{1 9 b} \mathbf{2 0 b}$, 21b, 22b, 23b, 24b, 25b, 26b, 27b, 28b, 29b, 30b and 31b. HPLC traces are also included. 


\section{NMR Spectra for the Synthesized Compounds}

Page S19: ${ }^{1} \mathrm{H}$ NMR and ${ }^{13} \mathrm{C}$ NMR of compound 9

Page S20: ${ }^{1} \mathrm{H}$ NMR and ${ }^{13} \mathrm{C}$ NMR of compound $\mathbf{1 0}$

Page S21: ${ }^{1} \mathrm{H}$ NMR and ${ }^{13} \mathrm{C}$ NMR of compound 12

Page S22: ${ }^{1} \mathrm{H}$ NMR and ${ }^{13} \mathrm{C}$ NMR of compound $\mathbf{1 3}$

Page S23: ${ }^{1} \mathrm{H}$ NMR and ${ }^{13} \mathrm{C}$ NMR of compound 14

Page S24: ${ }^{1} \mathrm{H}$ NMR and ${ }^{13} \mathrm{C}$ NMR of compound 15

Page S25: ${ }^{1} \mathrm{H}$ NMR and ${ }^{13} \mathrm{C}$ NMR of compound $\mathbf{1 7}$

Page S26-S33: ${ }^{1} \mathrm{H}$ NMR ${ }^{13} \mathrm{C}$ NMR and 2D-NMRs and HRMS of compound 4a

Page S34-S35: ${ }^{1} \mathrm{H}$ NMR, ${ }^{13} \mathrm{C}$ NMR and HRMS of compound $\mathbf{4 b}$

Page S36: Comparison of ${ }^{1} \mathrm{H}$ NMR, ${ }^{13} \mathrm{C}$ NMR compounds of $\mathbf{4 a}$ and $\mathbf{4 b}$

Page S37: ${ }^{1} \mathrm{H}$ NMR, ${ }^{13} \mathrm{C}$ NMR compound 2

Page S38: ${ }^{1} \mathrm{H}$ NMR and ${ }^{13} \mathrm{C}$ NMR of compound 18b

Page S39: ${ }^{1} \mathrm{H}$ NMR and ${ }^{13} \mathrm{C}$ NMR of compound 19b

Page S40: ${ }^{1} \mathrm{H}$ NMR and ${ }^{13} \mathrm{C}$ NMR of compound 20b

Page S41: ${ }^{1} \mathrm{H}$ NMR and ${ }^{13} \mathrm{C}$ NMR of compound 21b

Page S42: ${ }^{1} \mathrm{H}$ NMR and ${ }^{13} \mathrm{C}$ NMR of compound 22b

Page S43: ${ }^{1} \mathrm{H}$ NMR and ${ }^{13} \mathrm{C}$ NMR of compound 23b

Page S44: ${ }^{1} \mathrm{H}$ NMR and ${ }^{13} \mathrm{C}$ NMR of compound 24b

Page S45: ${ }^{1} \mathrm{H}$ NMR and ${ }^{13} \mathrm{C}$ NMR of compound 25b

Page S46: ${ }^{1} \mathrm{H}$ NMR and ${ }^{13} \mathrm{C}$ NMR of compound 26b

Page S47: ${ }^{1} \mathrm{H}$ NMR and ${ }^{13} \mathrm{C}$ NMR of compound 27b

Page S48: Complete citation for reference 20 


\section{General Information}

All reactions dealing with air- or moisture-sensitive compounds were carried out in oven- or flame-dried reaction vessels under an argon or nitrogen atmosphere. All chemicals were purchased commercially and used as received unless otherwise noted. Solvents for non-aqueous reactions were dried as follows prior to use: THF was dried with $\mathrm{KOH}$ and subsequently distilled from sodium/benzophenone, and then from a potassium/sodium alloy $(\mathrm{K} / \mathrm{Na} 4: 1) ; \mathrm{CH}_{2} \mathrm{Cl}_{2}$ was distilled from $\mathrm{CaH}_{2} ; \mathrm{MeOH}$ was dried by refluxing with $\mathrm{Mg}$-turnings (5 $\mathrm{g} / \mathrm{L}$ ) and subsequent distillation. Silica gel (200-300) for purification and silica gel TLC $\left(\mathrm{F}_{254}\right)$ were purchased from Qing Dao Hai Yang Chemical Industry Co. of China.

${ }^{1} \mathrm{H}$ NMR spectra were recorded on a Varian Inova $300 \mathrm{MHz}$ spectrometer. ${ }^{13} \mathrm{C}$ NMR spectra were recorded on the same spectrometer, operating at $75 \mathrm{MHz}$. Mass spectra were obtained on a ZAB-HS (EI, $70 \mathrm{eV}$ ) spectrometer. The enantiomeric excesses of the products were determined by HPLC analysis on an HP 1100 HPLC using a Diacel Chiralcel OJ-H (Daicel, 4.6 x $250 \mathrm{~mm}$ ) column and UV/CD detectors.

\section{Synthesis of Thiourea-Oxazoline Ligand 4}

\section{Compound 9}

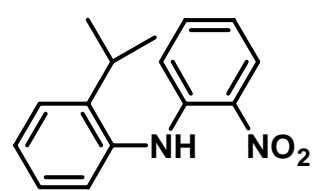

An oven-dried Schlenk flask was charged with $\mathrm{Cs}_{2} \mathrm{CO}_{3}$ (a finely ground powder, $9.12 \mathrm{~g}, 28$ $\mathrm{mmol}), \mathrm{Pd}(\mathrm{OAc})_{2}(89.8 \mathrm{mg}, 0.4 \mathrm{mmol})$ and BINAP $(373.6 \mathrm{mg}, 0.6 \mathrm{mmol})$ in a nitrogen-filled glove-box, and the bottle was capped with a rubber septum. To this flask were added 2-bromonitrobenzene $(4.1 \mathrm{~g}$, $20 \mathrm{mmol})$, 2-isopropylaniline $(3.4 \mathrm{~mL}, 24.0 \mathrm{mmol})$ and toluene $(40 \mathrm{~mL})$ and the reaction mixture was stirred at $115{ }^{\circ} \mathrm{C}$ for 2 days. After cooling to room temperature, the reaction mixture was diluted with ether $(50 \mathrm{~mL})$, and the mixture formed was filtered through a silica gel pad. The filtrate was concentrated in vacuo, and the residue was purified by a flash chromatography (hexane/ethyl acetate $=$ $40 / 1)$ on silica gel to give product $9(4.4 \mathrm{~g})$ in $85 \%$ yield; ${ }^{1} \mathrm{H}$ NMR $\left(300 \mathrm{MHz}, \mathrm{CDCl}_{3}\right): \delta 1.23(\mathrm{~d}, J=6.9$ $\mathrm{Hz}, 6 \mathrm{H}), 3.13-3.22(\mathrm{~m}, 1 \mathrm{H}), 6.70-6.75(\mathrm{~m}, 1 \mathrm{H}), 6.82-6.85(\mathrm{~m}, 1 \mathrm{H}), 7.26-7.35(\mathrm{~m}, 4 \mathrm{H}), 7.42-7.45(\mathrm{~m}, 1 \mathrm{H})$, 8.21-8.24 (m, 1H), $9.43(\mathrm{~s}, 1 \mathrm{H}) ;{ }^{13} \mathrm{C}$ NMR $\left(75 \mathrm{MHz}, \mathrm{CDCl}_{3}\right): \delta$ 23.2, 28.2, 115.8, 115.8, 116.7, 126.6, 126.8 , 126.8, 127.1, 127.4, 135.6, 135.8, 144.7, 145.2; HRMS (EI): calcd for $\mathrm{C}_{15} \mathrm{H}_{16} \mathrm{~N}_{2} \mathrm{O}_{2}\left(\mathrm{M}^{+}\right)$ 256.1212 , found 256.1209 .

\section{Compound 10}

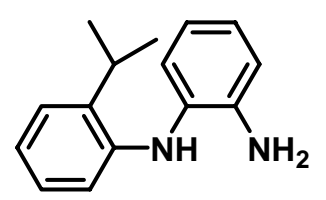

To a solution of compound $9(4.36 \mathrm{~g}, 17 \mathrm{mmol})$ in ethyl acetate $(20 \mathrm{~mL})$ was added palladium/carbon $(10 \%, 0.5 \mathrm{~g})$, and the solution was subjected to hydrogenation under a balloon pressure 
until the starting material was consumed. The catalyst was removed by filtration and the filtrate was concentrated in vacuo, and the residue was purified by a flash chromatography (hexane/ethyl acetate $=8 / 1$ ) on silica gel to give compound $10(3.66 \mathrm{~g})$ in $95 \%$ yield; ${ }^{1} \mathrm{H} \mathrm{NMR}\left(300 \mathrm{MHz}, \mathrm{CDCl}_{3}\right): \delta 1.31(\mathrm{~d}, J=6.9 \mathrm{~Hz}$, $6 \mathrm{H}), 3.05-3.14(\mathrm{~m}, 1 \mathrm{H}), 3.63(\mathrm{~s}, 2 \mathrm{H}), 5.16(\mathrm{~s}, 1 \mathrm{H}), 6.65-6.67(\mathrm{~m}, 1 \mathrm{H}), 6.73-6.81(\mathrm{~m}, 2 \mathrm{H}), 6.87-6.92(\mathrm{~m}$, $1 \mathrm{H}), 6.97-7.08(\mathrm{~m}, 3 \mathrm{H}), 7.22-7.27(\mathrm{~m}, 1 \mathrm{H}) ;{ }^{13} \mathrm{C} \mathrm{NMR}\left(75 \mathrm{MHz}, \mathrm{CDCl}_{3}\right): \delta 22.6,27.5,115.9,116.1,119.3$, 120.3, 124.0, 124.9, 125.5, 126.6, 129.8, 135.1, 141.1, 141.7; LRMS (EI): $226\left(\mathrm{M}^{+}\right)$; HRMS (EI): calcd for $\mathrm{C}_{15} \mathrm{H}_{18} \mathrm{~N}_{2}\left(\mathrm{M}^{+}\right)$226.1470, found 226.1470.

\section{Compound 12}

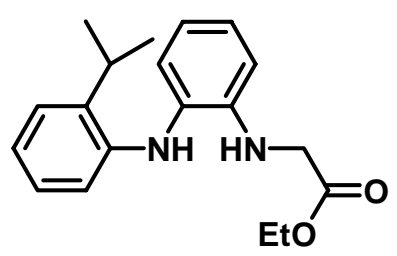

To a solution of compound $10(3.62 \mathrm{~g}, 16 \mathrm{mmol})$ and triethylamine $(3.6 \mathrm{~mL}, 25.6 \mathrm{mmol})$ in toluene $(100 \mathrm{~mL})$ was added ethyl bromoacetate $(2.5 \mathrm{~mL}, 22.4 \mathrm{mmol})$ in a dropwise manner at room temperature, and the reaction mixture was then stirred at $145^{\circ} \mathrm{C}$ (oil bath) for $1.5 \mathrm{~h}$. The reaction was worked up by addition of water $(50 \mathrm{~mL})$ after cooling to room temperature. The organic layer was separated, and the water phase was extracted with ethyl acetate $(3 \times 30 \mathrm{~mL})$, and the combined organic phases were dried over $\mathrm{Na}_{2} \mathrm{SO}_{4}$. The solvent was removed in vacuo and the residue was purified by flash chromatography (hexane/ethyl acetate $=8 / 1)$ on silica gel to give product $12(4.35 \mathrm{~g})$ in $87 \%$ yield.; ${ }^{1} \mathrm{H} \mathrm{NMR}(300 \mathrm{MHz}$, $\left.\mathrm{CDCl}_{3}\right): \delta 1.23(\mathrm{t}, 3 \mathrm{H}), 1.32(\mathrm{~d}, J=6.9 \mathrm{~Hz}, 6 \mathrm{H}), 3.05-3.14(\mathrm{~m}, 1 \mathrm{H}), 3.91(\mathrm{~s}, 2 \mathrm{H}), 4.16-4.23(\mathrm{~m}, 2 \mathrm{H}), 4.65$ $(\mathrm{s}, 1 \mathrm{H}), 5.20(\mathrm{~s}, 1 \mathrm{H}), 6.61-6.77(\mathrm{~m}, 3 \mathrm{H}), 6.87-6.92(\mathrm{~m}, 1 \mathrm{H}), 7.00-7.09(\mathrm{~m}, 3 \mathrm{H}), 7.25-7.27(\mathrm{~m}, 1 \mathrm{H}) ;{ }^{13} \mathrm{C}$ NMR (75 MHz, $\left.\mathrm{CDCl}_{3}\right): \delta 14.1,22.6,27.5,45.9,61.1,111.5,116.3,118.5,120.3,123.9,125.1,125.4$, 126.5, 130.2, 135.2, 142.0, 142.2, 171.0; HRMS (EI): calcd for $\mathrm{C}_{19} \mathrm{H}_{24} \mathrm{~N}_{2} \mathrm{O}_{2}\left(\mathrm{M}^{+}\right)$312.1838, found 312.1837 .

\section{Compound 13}

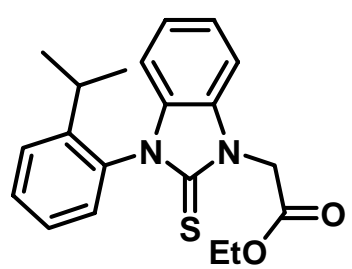

To a solution of $12(4.06 \mathrm{~g}, 13 \mathrm{mmol})$ and $\mathrm{NaHCO}_{3}(4.37 \mathrm{~g}, 52 \mathrm{mmol})$ in dry THF $(100 \mathrm{~mL})$ was added a solution of thiophosgene $(1.2 \mathrm{~mL}, 15.6 \mathrm{mmol})$ in THF $(20 \mathrm{~mL})$ at $50{ }^{\circ} \mathrm{C}$ in a dropwise manner, and the reaction mixture was stirred at the same temperature for $8 \mathrm{~h}$. The reaction was worked up by addition of water $(50 \mathrm{~mL})$, and the mixture was extracted with ethyl acetate $(3 \times 30 \mathrm{~mL})$. The combined organic layers were washed with brine $(2 \times 30 \mathrm{~mL})$, and dried over $\mathrm{Na}_{2} \mathrm{SO}_{4}$. The solvent was removed in vacuo and the residue was purified by flash chromatography (hexane/ethyl acetate $=8 / 1$ ) on silica gel to give product 13 (3.96 g) in 86\% yield; ${ }^{1} \mathrm{H} \mathrm{NMR}\left(300 \mathrm{MHz}, \mathrm{CDCl}_{3}\right): \delta 1.05(\mathrm{~d}, J=6.9 \mathrm{~Hz}, 3 \mathrm{H}), 1.24-1.31$ 
(m, 6H), 2.64-2.68 (m, 1H), 4.24-4.32 (m, 2H), $5.21(\mathrm{~d}, J=5.7 \mathrm{~Hz}, 2 \mathrm{H}), 6.76-6.79(\mathrm{~m}, 1 \mathrm{H})$, 7.13-7.20 (m, 2H), 7.24-7.29 (m, 2H), 7.37-7.42 (m, 1H), 7.55-7.57 (m, 2H); ${ }^{13} \mathrm{C}$ NMR (75 MHz, $\left.\mathrm{CDCl}_{3}\right): \delta 14.1,23.4$, 23.9, 28.2, 45.7, 61.9, 108.8, 109.9, 123.4, 123.4, 127.2, 127.2, 128.9, 130.3, 132.0, 133.1, 133.7, 147.4, 166.9, 171.7; HRMS (EI): calcd for $\mathrm{C}_{20} \mathrm{H}_{22} \mathrm{~N}_{2} \mathrm{O}_{2} \mathrm{~S}\left(\mathrm{M}^{+}\right)$354.1402, found 352.1404.

\section{Compound 14}

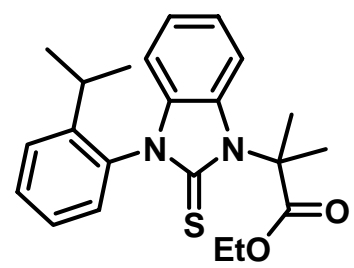

To a stirred solution of $13(3.54 \mathrm{~g}, 10.0 \mathrm{mmol})$ in dry THF $(50 \mathrm{~mL})$ was added LiHMDS (1 M in THF, $10 \mathrm{~mL}$ ) in a dropwise manner at $-78{ }^{\circ} \mathrm{C}$ under nitrogen, and mixture was stirred at the same temperature for $1 \mathrm{~h}$. To this solution was added MeI $(0.68 \mathrm{~mL}, 11.0 \mathrm{mmol})$ via syringe, and the mixture was stirred for $2 \mathrm{~h}$ at $-78^{\circ} \mathrm{C}$. The reaction mixture was quenched by addition of a saturated solution of $\mathrm{NH}_{4} \mathrm{Cl}(10 \mathrm{~mL})$, and the mixture was extracted with ethyl acetate $(3 \times 20 \mathrm{~mL})$, and the combined organic layers were dried over $\mathrm{Na}_{2} \mathrm{SO}_{4}$. The solvent was removed in vacuo and the residue was purified by a flash chromatography (hexane/ethyl acetate $=8 / 1$ ) on silica gel to afford the mono-methylation product after drying.

To a solution of the mono-methylation product, prepared above, in dry THF (50 mL) was added LiHMDS ( $1 \mathrm{M}$ in THF, $20 \mathrm{~mL}$ ) in a dropwise manner at $-78{ }^{\circ} \mathrm{C}$ under nitrogen, and the mixture was stirred at $0^{\circ} \mathrm{C}$ for $8 \mathrm{~h}$. To this solution was added MeI $(1.25 \mathrm{~mL}, 20 \mathrm{mmol})$ via syringe, and the mixture was stirred at room temperature for $8 \mathrm{~h}$. The reaction mixture was worked up by addition of a saturated solution of $\mathrm{NH}_{4} \mathrm{Cl}(30 \mathrm{~mL})$, and then extracted with ethyl acetate $(3 \times 30 \mathrm{~mL})$. The combined organic layers were dried over $\mathrm{Na}_{2} \mathrm{SO}_{4}$. The solvent was removed in vacuo and the residue was purified by flash chromatography (hexane/ethyl acetate $=8 / 1)$ on silica gel to give product $14(2.9 \mathrm{~g})$ in $76 \%$ yield for two steps; ${ }^{1} \mathrm{H}$ NMR $\left(300 \mathrm{MHz}, \mathrm{CDCl}_{3}\right): \delta 1.04(\mathrm{~d}, J=6.9 \mathrm{~Hz}, 3 \mathrm{H}), 1.20-1.25(\mathrm{~m}, 6 \mathrm{H}), 2.13(\mathrm{~s}, 3 \mathrm{H}), 2.19(\mathrm{~s}$, $3 \mathrm{H}), 2.56-2.65(\mathrm{~m}, 1 \mathrm{H}), 4.12-4.29(\mathrm{~m}, 2 \mathrm{H}), 6.70-6.73(\mathrm{~m}, 1 \mathrm{H}), 7.11-7.22(\mathrm{~m}, 3 \mathrm{H}), 7.35-7.40(\mathrm{~m}, 1 \mathrm{H})$, 7.50-7.55 (m, 2H), 7.67-7.69 (m, 1H); ${ }^{13} \mathrm{C}$ NMR (75 MHz, $\left.\mathrm{CDCl}_{3}\right): \delta 13.9,23.3,23.9,24.9,26.5,28.2$, 61.6, 65.7, 110.0, 111.7, 122.6, 122.7, 127.2, 127.2, 128.9, 130.2, 132.1, 133.2, 134.4, 147.3, 171.1, 173.0; HRMS (EI): calcd for $\mathrm{C}_{22} \mathrm{H}_{26} \mathrm{~N}_{2} \mathrm{O}_{2} \mathrm{~S}\left(\mathrm{M}^{+}\right)$382.1715, found 382.1712 .

\section{Compound 15}

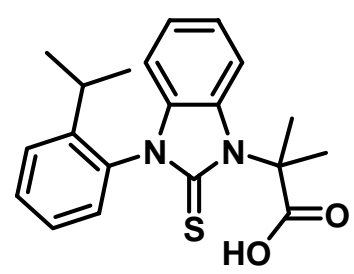

To a stirred suspension of potassium tert-butoxide $(5.83 \mathrm{~g}, 52 \mathrm{mmol})$ in dry THF $(100 \mathrm{~mL})$ was added water $(0.24 \mathrm{~mL}, 13.4 \mathrm{mmol})$ via syringe at $0{ }^{\circ} \mathrm{C}$, and the formed slurry was stirred for $5 \mathrm{~min}$. To this slurry was added compound $14(2.29 \mathrm{~g}, 6.0 \mathrm{mmol})$, and then the mixture was warmed to room 
temperature and stirred until the reaction was complete as monitored by TLC. The reaction was worked up by addition of ice water until two clear layers formed, the aqueous layer was separated and acidified with hydrochloric acid (2M).The acidified solution was first extracted with ethyl acetate $(3 \times 50 \mathrm{~mL})$, and then the combined organic layers were dried over $\mathrm{Na}_{2} \mathrm{SO}_{4}$. The solvent was removed in vacuo to give the product $15(2.02 \mathrm{~g})$ in 95\% yield; ${ }^{1} \mathrm{H}$ NMR $\left(300 \mathrm{MHz}, \mathrm{CDCl}_{3}\right): \delta 0.98(\mathrm{~d}, J=6.9 \mathrm{~Hz}, 3 \mathrm{H}), 1.17(\mathrm{~d}, J=$ $6.9 \mathrm{~Hz}, 3 \mathrm{H}), 2.01(\mathrm{~d}, J=3.3 \mathrm{~Hz}, 6 \mathrm{H}), 2.47-2.56(\mathrm{~m}, 1 \mathrm{H}), 6.63-6.68(\mathrm{~m}, 1 \mathrm{H}), 7.05-7.17(\mathrm{~m}, 3 \mathrm{H}), 7.24-7.31$ $(\mathrm{m}, 2 \mathrm{H}), 7.40-7.49(\mathrm{~m}, 2 \mathrm{H}), 7.57-7.60(\mathrm{~m}, 1 \mathrm{H}), 9.35(\mathrm{~s}, 1 \mathrm{H}) ;{ }^{13} \mathrm{C} \mathrm{NMR}\left(75 \mathrm{MHz}, \mathrm{CDCl}_{3}\right): \delta 23.3,23.7$, 25.8, 28.1, 66.4, 109.7, 111.9, 122.4, 122.6, 127.0, 129.1, 129.9, 132.3, 133.3, 134.2, 147.3, 170.8, 177.2; LRMS (EI): $354\left(\mathrm{M}^{+}\right)$; HRMS (EI): calcd for $\mathrm{C}_{20} \mathrm{H}_{22} \mathrm{~N}_{2} \mathrm{O}_{2} \mathrm{~S}\left(\mathrm{M}^{+}\right)$354.1402, found 354.1400.

\section{Compound 17}

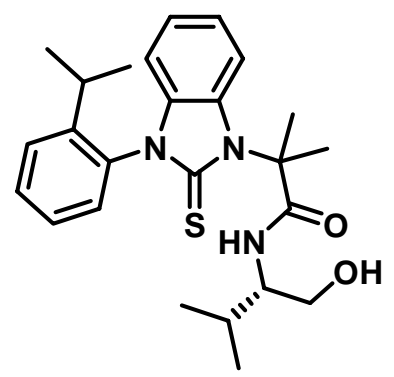

To a solution of compound $15(1.77 \mathrm{~g}, 5.0 \mathrm{mmol})$ and L-Valinol 16 (0.93 g, $9.0 \mathrm{mmol})$ in $\mathrm{CH}_{2} \mathrm{Cl}_{2}$ $(75 \mathrm{~mL})$ was added $\mathrm{EDC} \cdot \mathrm{HCl}(2.88 \mathrm{~g}, 15 \mathrm{mmol})$ in portions at $0{ }^{\circ} \mathrm{C}$. The mixture was first stirred at $0{ }^{\circ} \mathrm{C}$ for $30 \mathrm{~min}$ and then at room temperature for $12 \mathrm{~h}$. The reaction mixture was worked up by addition of $\mathrm{CH}_{2} \mathrm{Cl}_{2}(30 \mathrm{~mL})$, and then washed with brine $(2 \times 5 \mathrm{~mL})$, followed by $\mathrm{HCl}(1 \mathrm{M}, 5 \mathrm{~mL})$. The organic layer was dried over $\mathrm{Na}_{2} \mathrm{SO}_{4}$. The solvent was removed in vacuo and the residue was purified by flash chromatography (hexane/ $\mathrm{CH}_{2} \mathrm{Cl}_{2} /$ ethyl acetate $\left.=4 / 4 / 1\right)$ on silica gel to give product $17(1.98 \mathrm{~g})$ in $90 \%$ yield; ${ }^{1} \mathrm{H}$ NMR $\left(300 \mathrm{MHz}, \mathrm{CDCl}_{3}\right): \delta$ 0.85-0.96 (m, 6H), 1.02-1.05 (m, 3H), 1.24-1.26 (m, 3H), 1.80-1.88 $(\mathrm{m}, 1 \mathrm{H}), 2.15(\mathrm{~d}, J=3.6 \mathrm{~Hz}, 3 \mathrm{H}), 2.29(\mathrm{~d}, J=2.4 \mathrm{~Hz}, 3 \mathrm{H}), 2.44-2.58(\mathrm{~m}, 1 \mathrm{H}), 2.82(\mathrm{~s}, 1 \mathrm{H}), 3.64-3.60(\mathrm{~m}$, $1 \mathrm{H}), 3.63-3.75(\mathrm{~m}, 1 \mathrm{H}), 3.80-3.89(\mathrm{~m}, 1 \mathrm{H}), 5.73-5.79(\mathrm{~m}, 1 \mathrm{H}), 6.73-6.77(\mathrm{~m}, 1 \mathrm{H}), 7.13-7.26(\mathrm{~m}, 3 \mathrm{H})$, 7.35-7.41 (m, 1H), 7.54-7.55 (m, 2H), 7.45-7.78 (m, 1H); ${ }^{13} \mathrm{C}$ NMR (75 MHz, $\left.\mathrm{CDCl}_{3}\right): \delta 19.1,19.4,19.6$, 19.7, 23.2, 23.2, 23.8, 24.0, 25.1, 25.1, 27.8, 28.3, 28.5, 28.8, 28.9, 57.6, 57.9, 61.4, 62.3, 67.7, 110.4, $110.4,112.5,112.5,123.2,123.3,127.2,127.3,127.3,127.4,128.7,129.0,130.3,130.4,132.3,132.3$, $132.9,134.5,134.6,147.0,147.2,170.9,171.0,173.0 ; \mathrm{MS} \mathrm{m} / \mathrm{z}(\mathrm{EI}): 439\left(\mathrm{M}^{+}\right)$.

\section{The Pd-Catalyzed Asymmetric Bis-methoxycarbonylations of Terminal Olefins}

General Procedure for the Pd-catalyzed asymmetric bis-methoxycarbonylations of terminal olefins: To $\mathrm{MeOH}(4.0 \mathrm{~mL})$ in an oven-dried Schlenk tube were added $\mathrm{Pd}\left[\left(\mathrm{C}_{3} \mathrm{H}_{5}\right) \mathrm{Cl}\right]_{2}(5.5 \mathrm{mg}, 0.015 \mathrm{mmol})$, ligand $4 \mathbf{a}(25.3 \mathrm{mg}, 0.06 \mathrm{mmol}), \mathrm{CuCl}(20 \mathrm{mg}, 0.2 \mathrm{mmol})$, and the mixture was stirred at $20{ }^{\circ} \mathrm{C}$ for $30 \mathrm{~min}$ under nitrogen. To this solution was added terminal olefins $(1.0 \mathrm{mmol})$ via syringe and the mixture was stirred at $20{ }^{\circ} \mathrm{C}$ under a balloon pressure of $\mathrm{CO}$ and $\mathrm{O}_{2}$ (ca 4:1), for an additional $52 \mathrm{~h}$. The solvent was 
removed in vacuo and the residue was purified by flash chromatography (hexane/ethyl acetate $=8 / 1$ ) on silica gel to give the corresponding products listed below.

\section{Compound 2 (Table 3, entry 7)}<smiles>COC(=O)CC(C(C)=O)c1ccccc1</smiles>

Product 2 was obtained in 95\% yield with 75\% ee; ${ }^{1} \mathrm{H}$ NMR $\left(300 \mathrm{MHz}, \mathrm{CDCl}_{3}\right): \delta 2.64-2.71(\mathrm{~m}$, $1 \mathrm{H}), 3.17-3.26(\mathrm{~m}, 1 \mathrm{H}), 3.64(\mathrm{~s}, 3 \mathrm{H}), 3.68(\mathrm{~s}, 3 \mathrm{H}), 4.08-4.13(\mathrm{~m}, 1 \mathrm{H}), 7.25-7.34(\mathrm{~m}, 5 \mathrm{H}) ;{ }^{13} \mathrm{C}$ NMR $(75$ $\left.\mathrm{MHz}, \mathrm{CDCl}_{3}\right): \delta 37.5,46.9,51.8,52.3,127.6,127.6,128.8,137.6,171.9,173.3$; HRMS (EI): calcd for $\mathrm{C}_{12} \mathrm{H}_{14} \mathrm{O}_{4}\left(\mathrm{M}^{+}\right)$222.0892, found 222.0894; HPLC conditions: CHIRALCEL OJ-H, hexane/i-PrOH = $90 / 10,0.8 \mathrm{~mL} / \mathrm{min}, 230 \mathrm{~nm}, 30^{\circ} \mathrm{C}, \mathrm{t}_{\mathrm{r}}$ (major) $=19.9 \mathrm{~min}, \mathrm{t}_{\mathrm{r}}($ minor $)=24.8 \mathrm{~min}$.
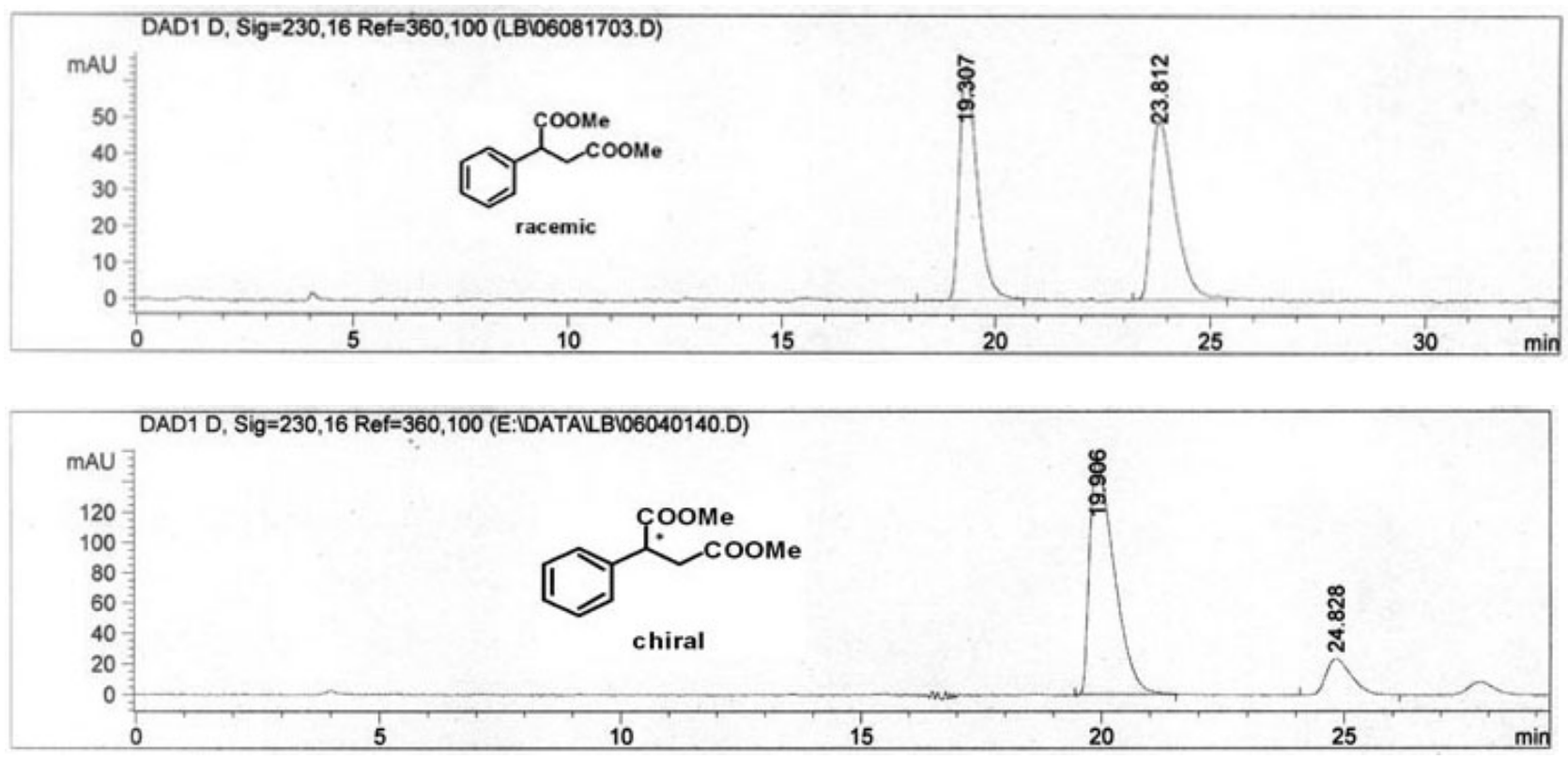

\section{Compound 18b (Table 4, entry 1)}<smiles>CCOc1ccc(C(CC(C)=O)C(C)=O)cc1</smiles>

Product 18b was obtained in 96\% yield with 65\% ee; ${ }^{1} \mathrm{H}$ NMR $\left(300 \mathrm{MHz}, \mathrm{CDCl}_{3}\right): \delta 1.39(\mathrm{t}, 3 \mathrm{H})$, 2.61-2.68 (m, 1H), 3.13-3.22 (m, 1H), $3.66(\mathrm{~s}, 6 \mathrm{H}), 3.97-4.06(\mathrm{~m}, 3 \mathrm{H}), 6.83-6.86(\mathrm{~m}, 2 \mathrm{H})$, 7.18-7.20 (m, 
$2 \mathrm{H}) ;{ }^{13} \mathrm{C} \mathrm{NMR}\left(75 \mathrm{MHz}, \mathrm{CDCl}_{3}\right): \delta 14.7,37.6,46.1,51.7,52.1,63.3,114.7,128.6,129.4,158.3,171.9$, 173.6; HRMS (EI): calcd for $\mathrm{C}_{14} \mathrm{H}_{18} \mathrm{O}_{5}\left(\mathrm{M}^{+}\right)$266.1154, found 266.1151; HPLC conditions: CHIRALCEL $\mathrm{OJ}-\mathrm{H}$, hexane $/ \mathrm{i}-\mathrm{PrOH}=90 / 10,0.8 \mathrm{~mL} / \mathrm{min}, 230 \mathrm{~nm}, 30{ }^{\circ} \mathrm{C}, \mathrm{t}_{\mathrm{r}}($ minor $)=17.6 \mathrm{~min}, \mathrm{t}_{\mathrm{r}}($ major$)=20.7 \mathrm{~min}$.
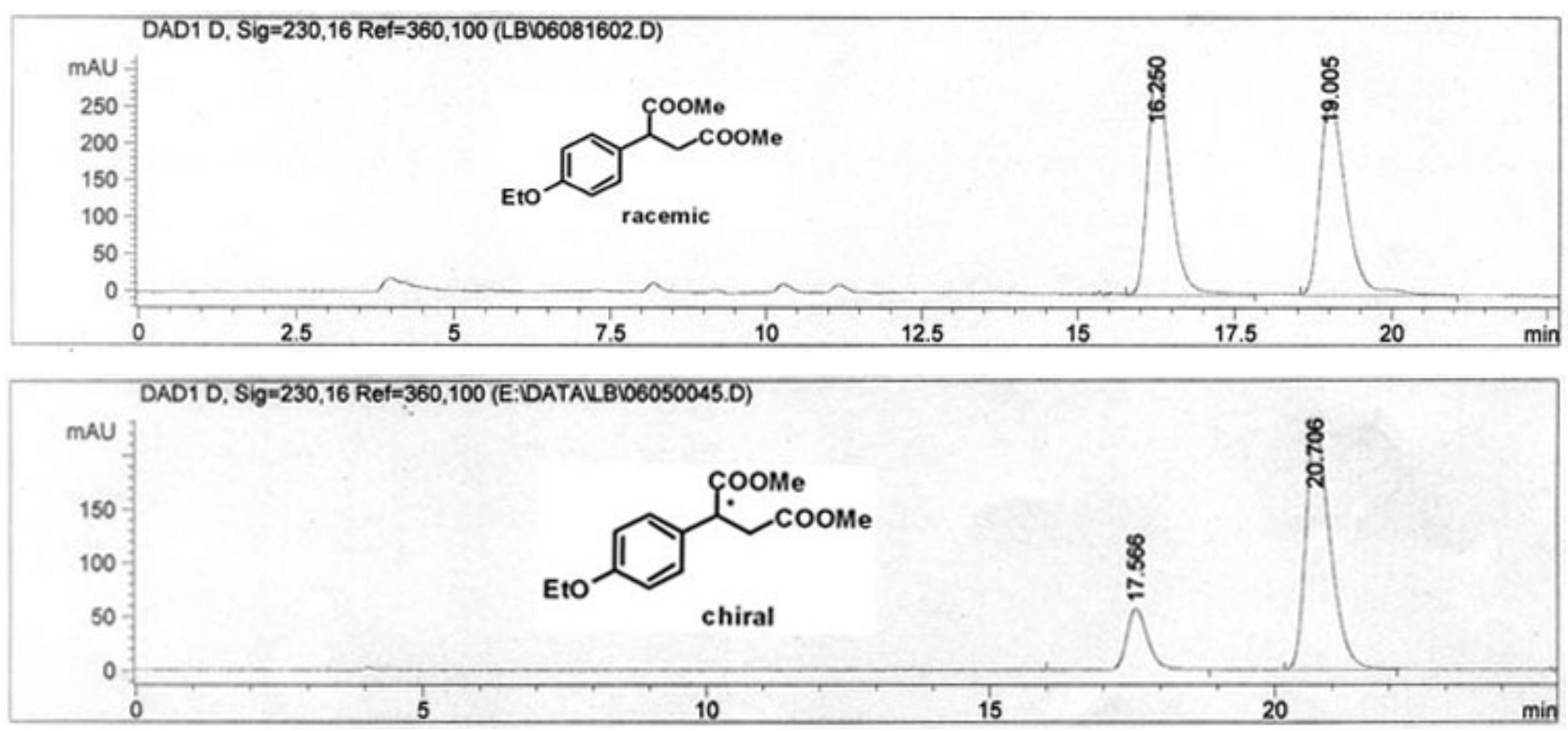

\section{Compound 19b (Table 4, entry 2)}<smiles>COC(=O)CC(C(C)=O)c1ccc(OC)cc1</smiles>

Product 19b was obtained in 93\% yield with $65 \% \mathrm{ee} ;{ }^{1} \mathrm{H}$ NMR $\left(300 \mathrm{MHz}, \mathrm{CDCl}_{3}\right): \delta 2.61-2.69(\mathrm{~m}$, $1 \mathrm{H}), 3.13-3.23(\mathrm{~m}, 1 \mathrm{H}), 3.67(\mathrm{~s}, 3 \mathrm{H}), 3.70(\mathrm{~s}, 3 \mathrm{H}), 3.81(\mathrm{~s}, 3 \mathrm{H}), 4.02-4.07(\mathrm{~m}, 1 \mathrm{H}), 6.85-6.89(\mathrm{~m}, 2 \mathrm{H})$, 7.19-7.22 (m, 2H); ${ }^{13} \mathrm{C}$ NMR (75 MHz, $\left.\mathrm{CDCl}_{3}\right): \delta 37.6,46.1,51.7,52.2,55.1,114.1,128.7,129.6,158.9$, 171.9, 173.6; HRMS (EI): calcd for $\mathrm{C}_{13} \mathrm{H}_{16} \mathrm{O}_{5}\left(\mathrm{M}^{+}\right)$252.0998, found 252.1000; HPLC conditions: CHIRALCEL OJ-H, hexane $/ \mathrm{i}-\mathrm{PrOH}=90 / 10,0.8 \mathrm{~mL} / \mathrm{min}, 230 \mathrm{~nm}, 30{ }^{\circ} \mathrm{C}, \mathrm{t}_{\mathrm{r}}$ (minor) $=26.4 \mathrm{~min}$, $\mathrm{t}_{\mathrm{r}}$ (major) $=29.6 \mathrm{~min}$.

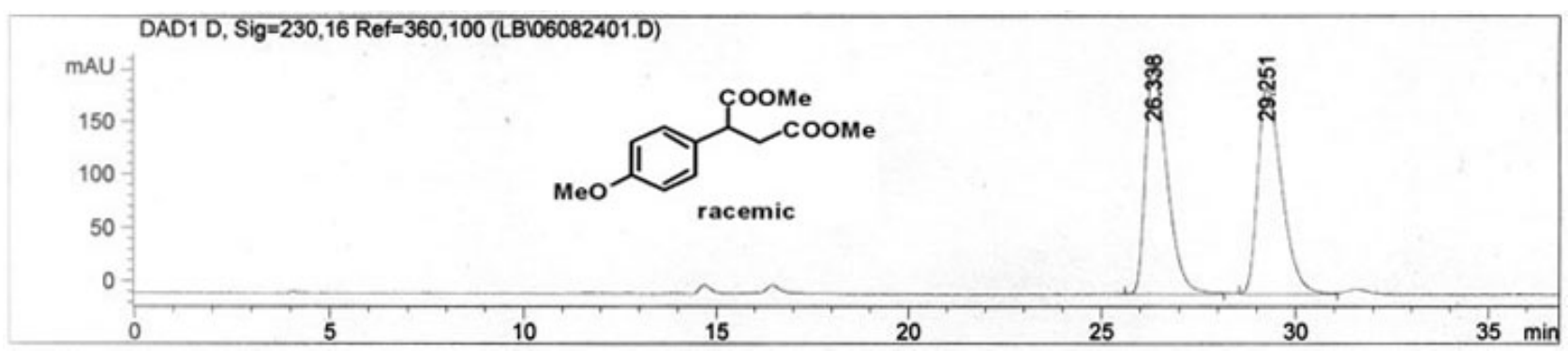




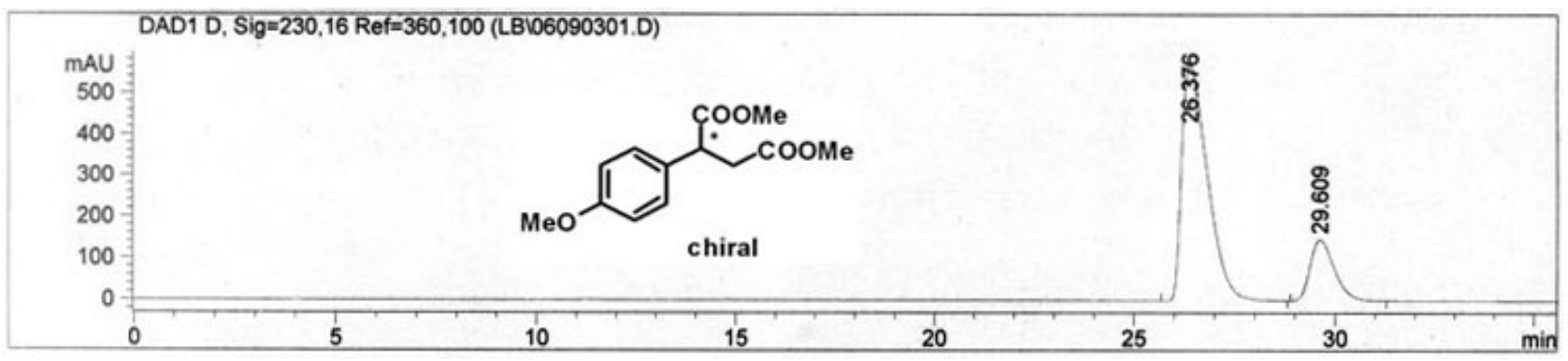

\section{Compound 20b (Table 4, entry 3)}<smiles>COC(=O)CC(C(C)=O)c1ccc(C)cc1</smiles>

Product 20b was obtained in 95\% yield with $65 \%$ ee; ${ }^{1} \mathrm{H}$ NMR $\left(300 \mathrm{MHz}, \mathrm{CDCl}_{3}\right): \delta 2.32(\mathrm{~s}, 3 \mathrm{H})$, 2.61-2.68 (m, 1H), 3.15-3.24 (m, 1H), $3.67(\mathrm{~s}, 6 \mathrm{H}), 4.04-4.09(\mathrm{~m}, 1 \mathrm{H}), 7.12-7.19(\mathrm{~m}, 4 \mathrm{H}) ;{ }^{13} \mathrm{C} \mathrm{NMR}(75$ $\mathrm{MHz}, \mathrm{CDCl}_{3}$ ): $\delta 20.8,37.4,46.5,51.6,52.1,127.4,129.4,134.5,137.2,171.8,173.4$; HRMS (EI): calcd for $\mathrm{C}_{13} \mathrm{H}_{16} \mathrm{O}_{4}\left(\mathrm{M}^{+}\right)$236.1049, found 236.1047; HPLC conditions: CHIRALCEL OJ-H, hexane/i-PrOH = $90 / 10,0.8 \mathrm{~mL} / \mathrm{min}, 230 \mathrm{~nm}, 30^{\circ} \mathrm{C}, \mathrm{t}_{\mathrm{r}}$ (minor) $=14.4 \mathrm{~min}, \mathrm{t}_{\mathrm{r}}$ (major) $=18.0 \mathrm{~min}$.
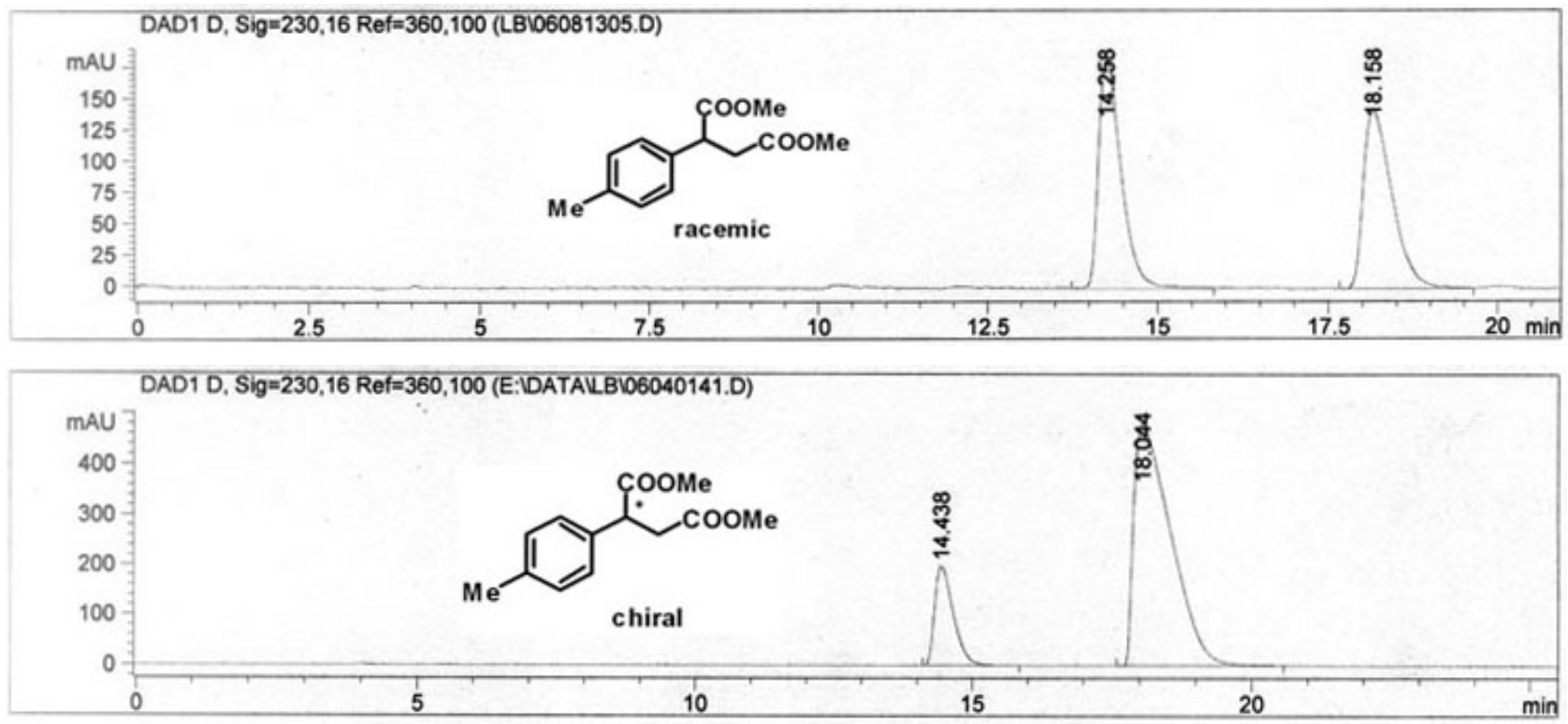


\section{Compound 21b (Table 4, entry 4)}<smiles>COC(=O)CC(C(C)=O)c1ccccc1C</smiles>

Product 21b was obtained in 76\% yield with $60 \%$ ee; ${ }^{1} \mathrm{H}$ NMR $\left(300 \mathrm{MHz}, \mathrm{CDCl}_{3}\right): \delta 2.43(\mathrm{~s}, 3 \mathrm{H})$, 2.57-2.64 (m, 1H), $3.67(\mathrm{~s}, 3 \mathrm{H}), 3.68(\mathrm{~s}, 3 \mathrm{H}), 4.35-4.40(\mathrm{~m}, 1 \mathrm{H}), 7.15-7.21(\mathrm{~m}, 4 \mathrm{H}) ;{ }^{13} \mathrm{C}$ NMR $(75 \mathrm{MHz}$, $\left.\mathrm{CDCl}_{3}\right): \delta 19.5,36.9,42.6,51.7,52.0,126.4,126.5,127.3,130.7,135.9,136.1,171.9,173.6$; HRMS (EI): calcd for $\mathrm{C}_{13} \mathrm{H}_{16} \mathrm{O}_{4}\left(\mathrm{M}^{+}\right)$236.1049, found 236.1047; HPLC conditions: CHIRALCEL OJ-H, hexane $/ \mathrm{i}-\mathrm{PrOH}=90 / 10,0.8 \mathrm{~mL} / \mathrm{min}, 230 \mathrm{~nm}, 30{ }^{\circ} \mathrm{C}, \mathrm{t}_{\mathrm{r}}$ (minor) $=13.2 \mathrm{~min}, \mathrm{t}_{\mathrm{r}}$ (major) $=15.2 \mathrm{~min}$.
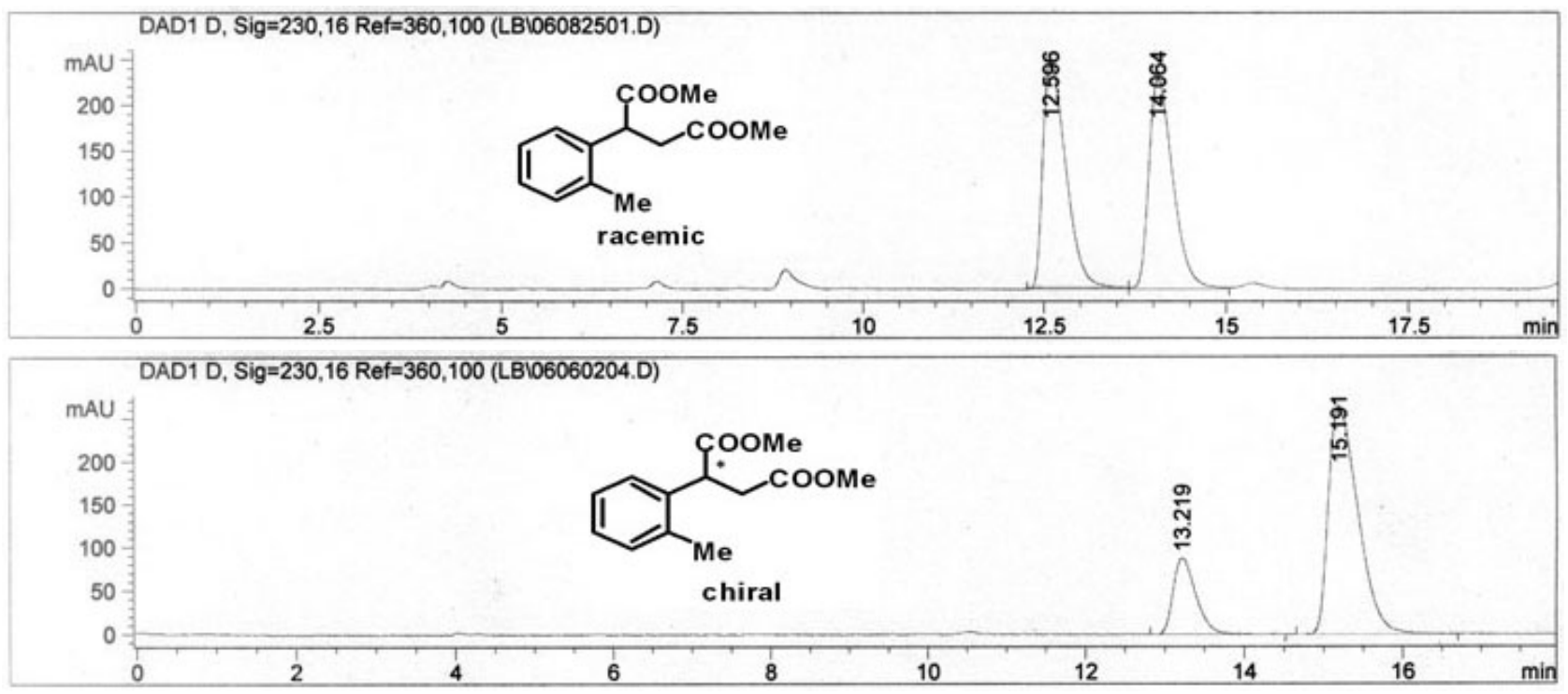

\section{Compound 22b (Table 4, entry 5)}<smiles>COC(=O)CC(C(C)=O)c1ccc(C)cc1C</smiles>

Product 22b was obtained in 94\% yield with $60 \%$ ee; ${ }^{1} \mathrm{H}$ NMR $\left(300 \mathrm{MHz}, \mathrm{CDCl}_{3}\right): \delta 2.27(\mathrm{~s}, 3 \mathrm{H})$, $2.38(\mathrm{~s}, 3 \mathrm{H}), 2.54-2.61(\mathrm{~m}, 1 \mathrm{H}), 3.12-3.21(\mathrm{~m}, 1 \mathrm{H}), 3.65(\mathrm{~s}, 3 \mathrm{H}), 3.68(\mathrm{~s}, 3 \mathrm{H}), 4.31-4.36(\mathrm{~m}, 1 \mathrm{H})$, 6.96-7.08 (m, 3H); ${ }^{13} \mathrm{C}$ NMR $\left(75 \mathrm{MHz}, \mathrm{CDCl}_{3}\right): \delta 19.36,20.68,36.86,42.21,51.55,51.98,126.39$, 127.01, 131.38, 133.08, 135.65, 136.85, 171.96, 173.69; LRMS (EI): 250 ( $\left.{ }^{+}\right)$; HRMS (EI): calcd for $\mathrm{C}_{14} \mathrm{H}_{18} \mathrm{O}_{4}\left(\mathrm{M}^{+}\right)$250.1205, found 250.1208; HPLC conditions: CHIRALCEL OJ-H, hexane/i-PrOH = $90 / 10,0.8 \mathrm{~mL} / \mathrm{min}, 230 \mathrm{~nm}, 30^{\circ} \mathrm{C}, \mathrm{t}_{\mathrm{r}}($ minor $)=9.0 \mathrm{~min}, \mathrm{t}_{\mathrm{r}}($ major $)=12.9 \mathrm{~min}$. 

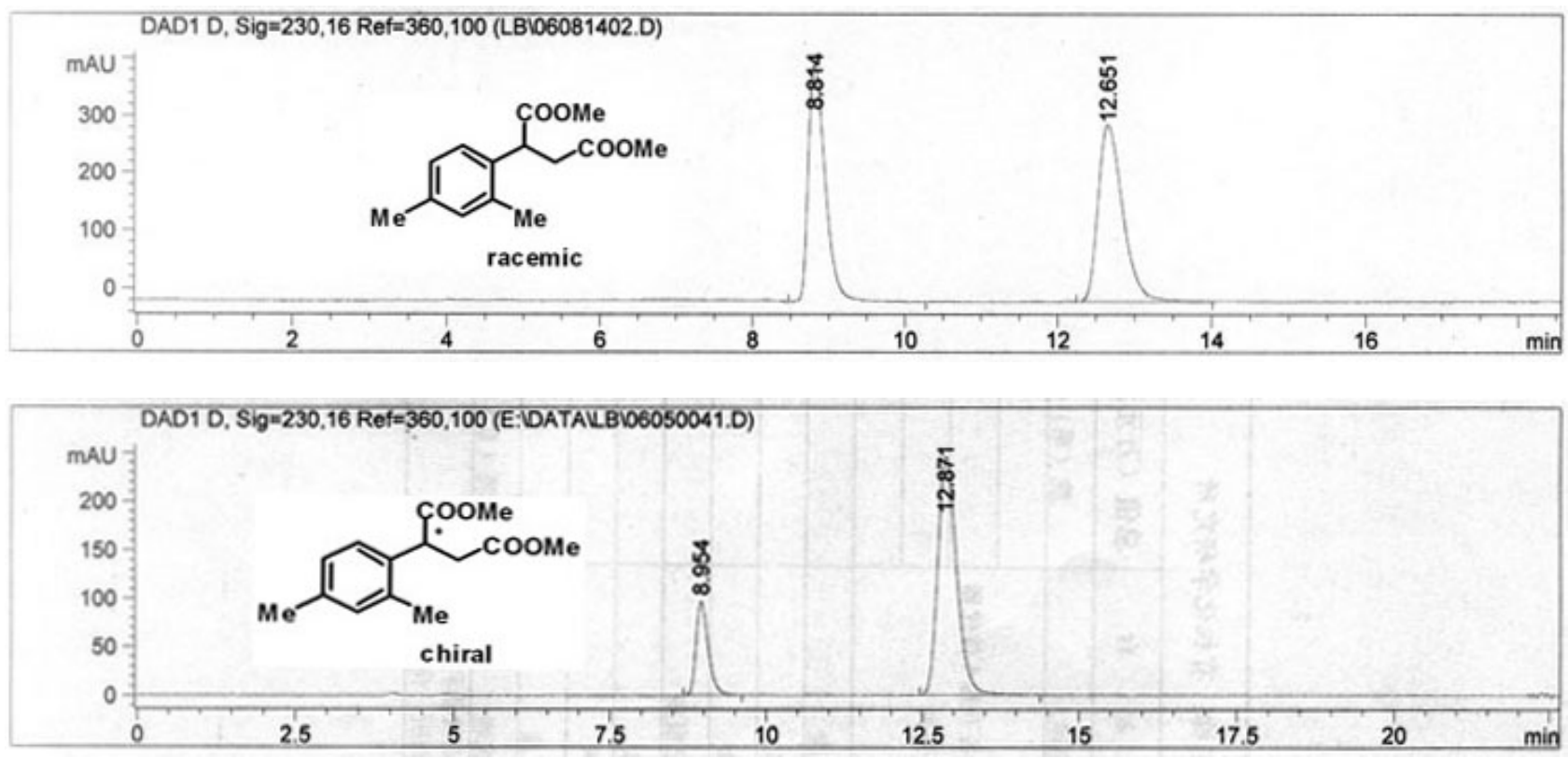

\section{Compound 23b (Table 4, entry 6)}<smiles>COC(=O)CC(C(C)=O)c1ccc(F)cc1</smiles>

Product 23b was obtained in 92\% yield with $55 \%$ ee; ${ }^{1} \mathrm{H}$ NMR $\left(300 \mathrm{MHz}, \mathrm{CDCl}_{3}\right): \delta 2.63-2.70(\mathrm{~m}$, $1 \mathrm{H}), 3.14-3.23(\mathrm{~m}, 1 \mathrm{H}), 3.68(\mathrm{~s}, 3 \mathrm{H}), 3.69(\mathrm{~m}, 3 \mathrm{H}), 4.06-4.11(\mathrm{~m}, 1 \mathrm{H}), 7.00-7.05(\mathrm{~m}, 2 \mathrm{H}), 7.24-7.29$ (m, $2 \mathrm{H}) ;{ }^{13} \mathrm{C}$ NMR $\left(75 \mathrm{MHz}, \mathrm{CDCl}_{3}\right): \delta 37.6,46.3,51.8,52.4,115.5,115.9,129.3,129.4,133.3,133.4,159.8$, 164.7, 171.7, 173.2; HRMS (EI): calcd for $\mathrm{C}_{12} \mathrm{H}_{13} \mathrm{O}_{4} \mathrm{~F}\left(\mathrm{M}^{+}\right)$240.0798, found 240.0810; HPLC conditions: CHIRALCEL OJ-H, hexane $/ \mathrm{i}-\mathrm{PrOH}=90 / 10,0.8 \mathrm{~mL} / \mathrm{min}, 230 \mathrm{~nm}, 30{ }^{\circ} \mathrm{C}, \mathrm{t}_{\mathrm{r}}$ (minor) $=14.4 \mathrm{~min}, \mathrm{t}_{\mathrm{r}}$ (major) $=15.2 \mathrm{~min}$.

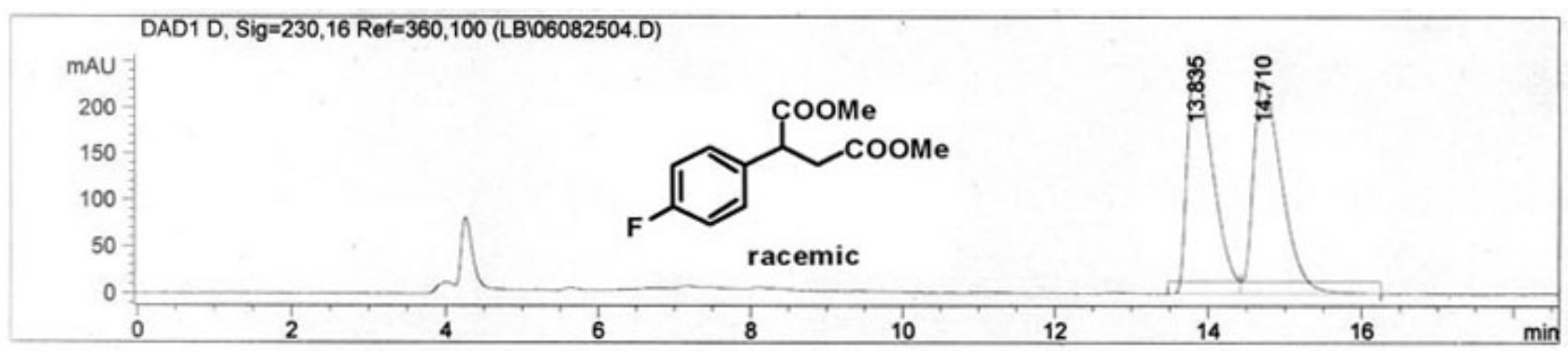




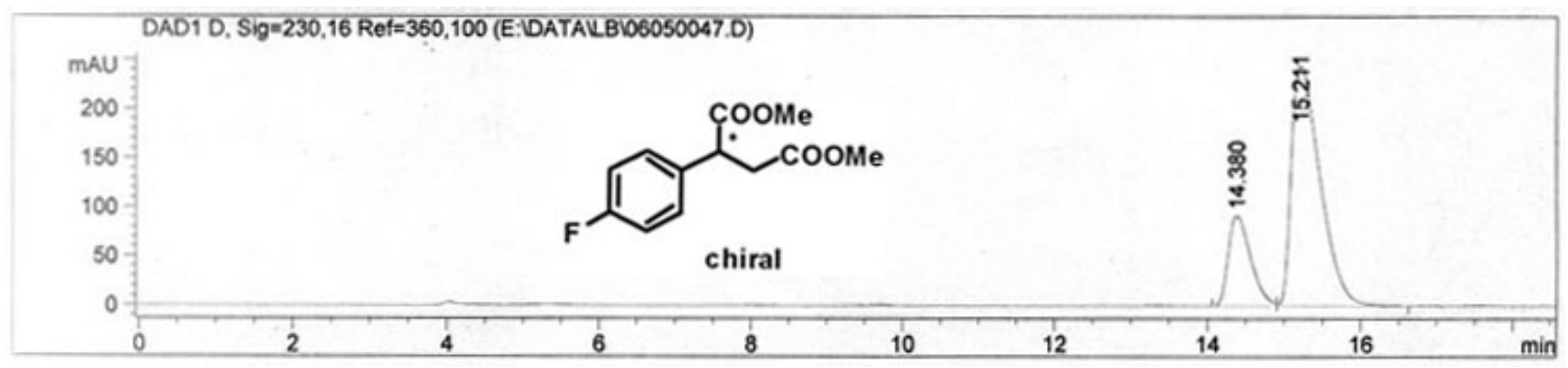

\section{Compound 24b (Table 4, entry 7)}<smiles>COC(=O)CC(C(C)=O)c1ccc(Cl)cc1</smiles>

Product 24b was obtained in 95\% yield with $46 \%$ ee; ${ }^{1} \mathrm{H}$ NMR $\left(300 \mathrm{MHz}, \mathrm{CDCl}_{3}\right): \delta 2.63-2.70(\mathrm{~m}$, $1 \mathrm{H}), 3.14-3.23(\mathrm{~m}, 1 \mathrm{H}), 3.67(\mathrm{~s}, 3 \mathrm{H}), 3.68(\mathrm{~s}, 3 \mathrm{H}), 4.05-4.10(\mathrm{~m}, 1 \mathrm{H}), 7.22-7.25(\mathrm{~m}, 2 \mathrm{H}), 7.29-7.32(\mathrm{~m}$, $2 \mathrm{H}) ;{ }^{13} \mathrm{C} \mathrm{NMR}\left(75 \mathrm{MHz}, \mathrm{CDCl}_{3}\right): \delta 37.3,46.4,51.9,52.4,128.9,129.1,133.6,136.0,171.6,172.9$; HRMS (EI): calcd for $\mathrm{C}_{12} \mathrm{H}_{13} \mathrm{O}_{4}{ }^{35} \mathrm{Cl}\left(\mathrm{M}^{+}\right)$256.0502, found 256.0503; HPLC conditions: CHIRALCEL $\mathrm{OJ}-\mathrm{H}$, hexane $/ \mathrm{i}-\mathrm{PrOH}=90 / 10,0.8 \mathrm{~mL} / \mathrm{min}, 230 \mathrm{~nm}, 30{ }^{\circ} \mathrm{C}, \mathrm{t}_{\mathrm{r}}$ (minor) $=13.4 \mathrm{~min}, \mathrm{t}_{\mathrm{r}}$ (major) $=14.8 \mathrm{~min}$.
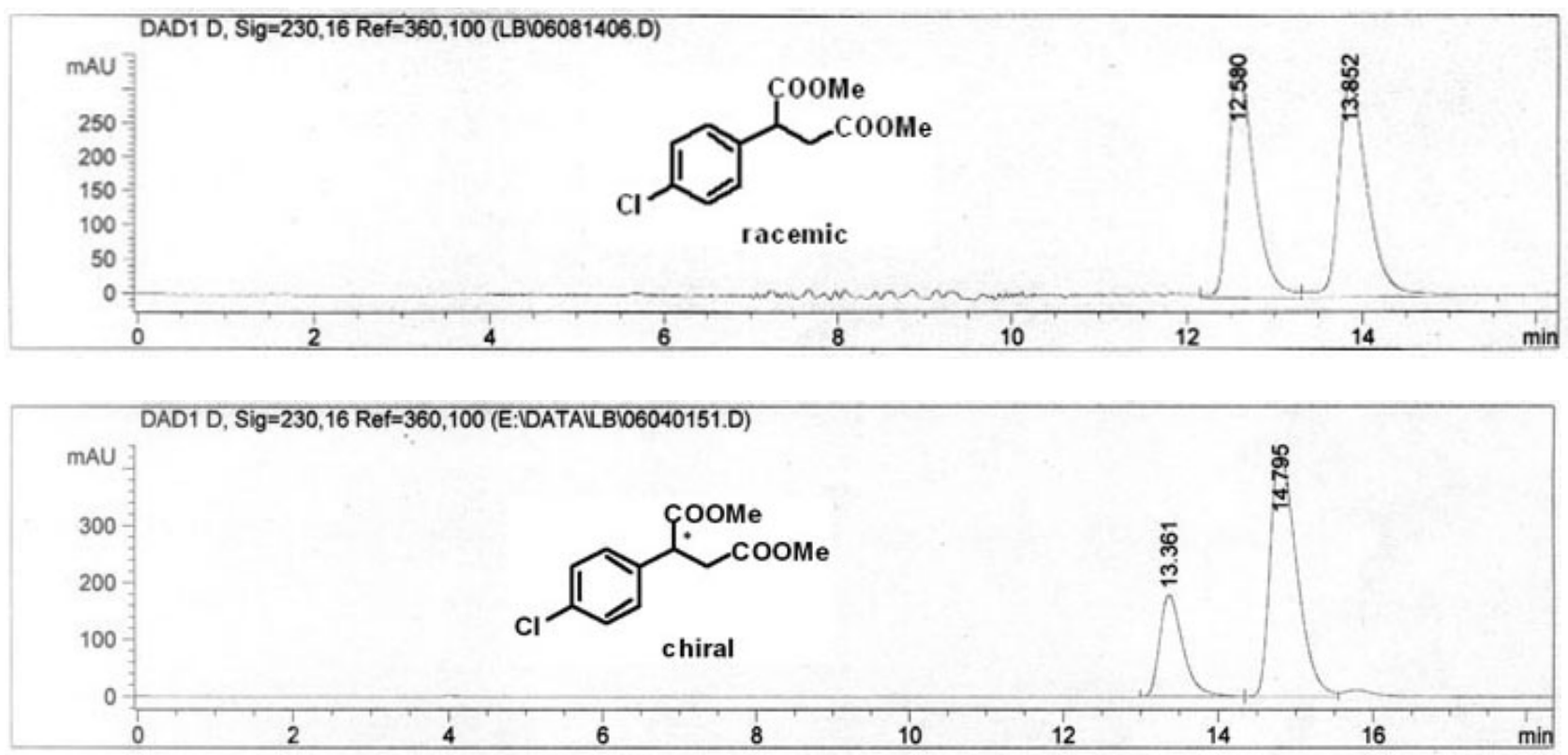

\section{Compound 25b (Table 4, entry 8)}


<smiles>COC(=O)CC(C(C)=O)c1ccc(Br)cc1</smiles>

Product 25b was obtained in 92\% yield with $44 \%$ ee; ${ }^{1} \mathrm{H}$ NMR $\left(300 \mathrm{MHz}, \mathrm{CDCl}_{3}\right): \delta 2.62-2.70(\mathrm{~m}$, $1 \mathrm{H}), 3.13-3.22(\mathrm{~m}, 1 \mathrm{H}), 3.68(\mathrm{~s}, 3 \mathrm{H}), 3.71(\mathrm{~s}, 3 \mathrm{H}), 4.04-4.09(\mathrm{~m}, 1 \mathrm{H}), 7.16-7.20(\mathrm{~m}, 2 \mathrm{H})$, 7.45-7.49 (m, $2 \mathrm{H}) ;{ }^{13} \mathrm{C} \mathrm{NMR}\left(75 \mathrm{MHz}, \mathrm{CDCl}_{3}\right): \delta 37.2,46.4,51.8,52.3,121.6,129.4,131.9,136.5,171.5,172.8$; HRMS (EI): calcd for $\mathrm{C}_{12} \mathrm{H}_{13} \mathrm{O}_{4}{ }^{79} \mathrm{Br}\left(\mathrm{M}^{+}\right)$299.9997, found 299.9985; HPLC conditions: CHIRALCEL $\mathrm{OJ}-\mathrm{H}$, hexane $/ \mathrm{i}-\mathrm{PrOH}=90 / 10,0.8 \mathrm{~mL} / \mathrm{min}, 230 \mathrm{~nm}, 30^{\circ} \mathrm{C}, \mathrm{t}_{\mathrm{r}}($ minor $)=13.7 \mathrm{~min}, \mathrm{t}_{\mathrm{r}}($ major$)=15.5 \mathrm{~min}$.
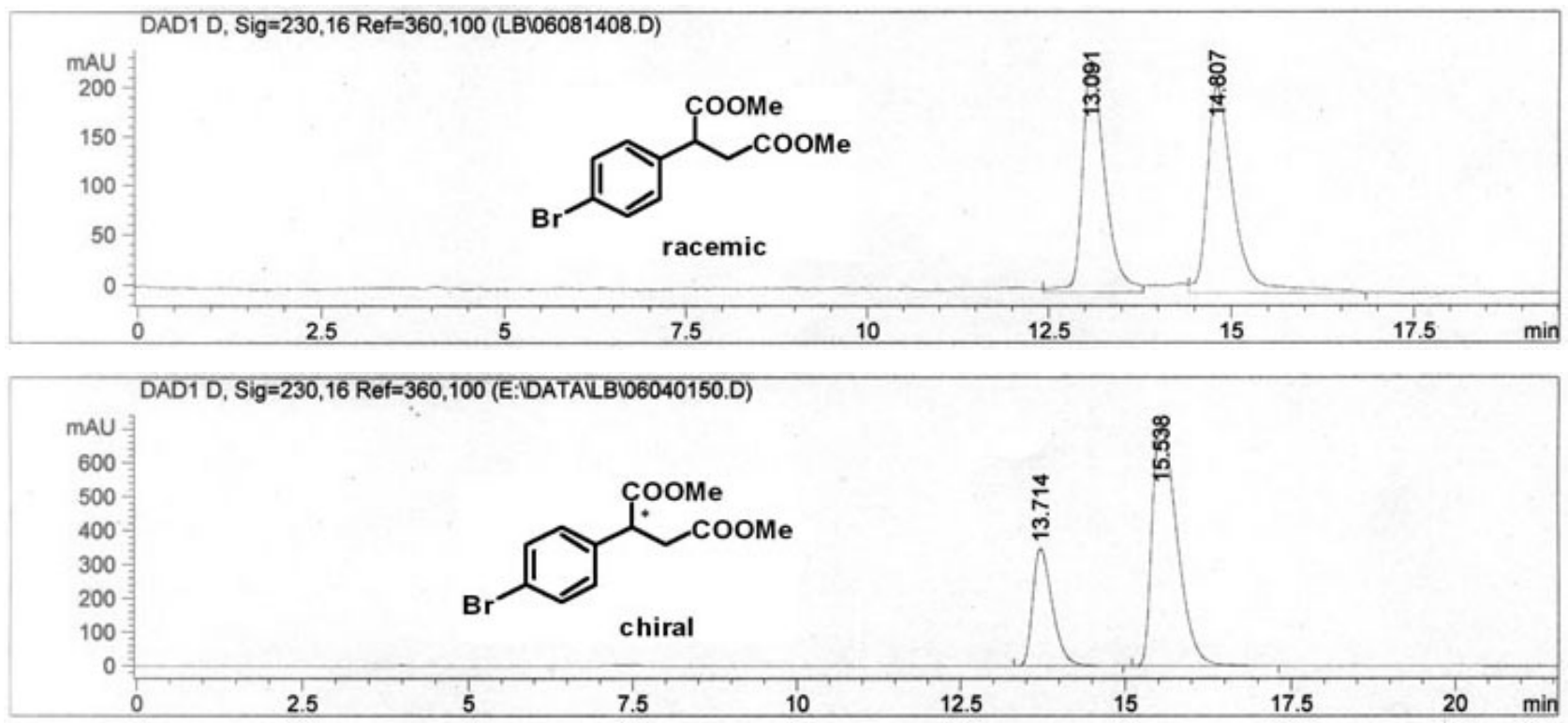

\section{Compound 26b (Table 4, entry 9)}<smiles>COC(C)=O</smiles>

Product 26b was obtained in $90 \%$ yield with $56 \%$ ee; ${ }^{1} \mathrm{H}$ NMR $\left(300 \mathrm{MHz}, \mathrm{CDCl}_{3}\right): \delta 2.72-2.79(\mathrm{~m}$, $1 \mathrm{H}), 3.27-3.36(\mathrm{~m}, 1 \mathrm{H}), 3.66(\mathrm{~s}, 3 \mathrm{H}), 3.67(\mathrm{~s}, 3 \mathrm{H}), 4.25-4.30(\mathrm{~m}, 1 \mathrm{H}), 7.38-7.47(\mathrm{~m}, 3 \mathrm{H}), 7.74-7.81(\mathrm{~m}$, $4 \mathrm{H}) ;{ }^{13} \mathrm{C} \mathrm{NMR}\left(75 \mathrm{MHz}, \mathrm{CDCl}_{3}\right): \delta 37.5,47.1,51.7,52.3,125.5,126.0,126.3,126.6,127.5,127.7,128.6$, 132.7, 133.3, 134.9, 171.8, 173.3; HPLC conditions: HRMS (EI): calcd for $\mathrm{C}_{16} \mathrm{H}_{16} \mathrm{O}_{4}\left(\mathrm{M}^{+}\right)$272.1049, found 272.1044; CHIRALCEL OJ-H, hexane $/ \mathrm{i}-\mathrm{PrOH}=90 / 10,0.8 \mathrm{~mL} / \mathrm{min}, 230 \mathrm{~nm}, 30^{\circ} \mathrm{C}, \mathrm{t}_{\mathrm{r}}$ (major) $=$ $33.7 \mathrm{~min}, \mathrm{t}_{\mathrm{r}}($ minor $)=36.7 \mathrm{~min}$. 

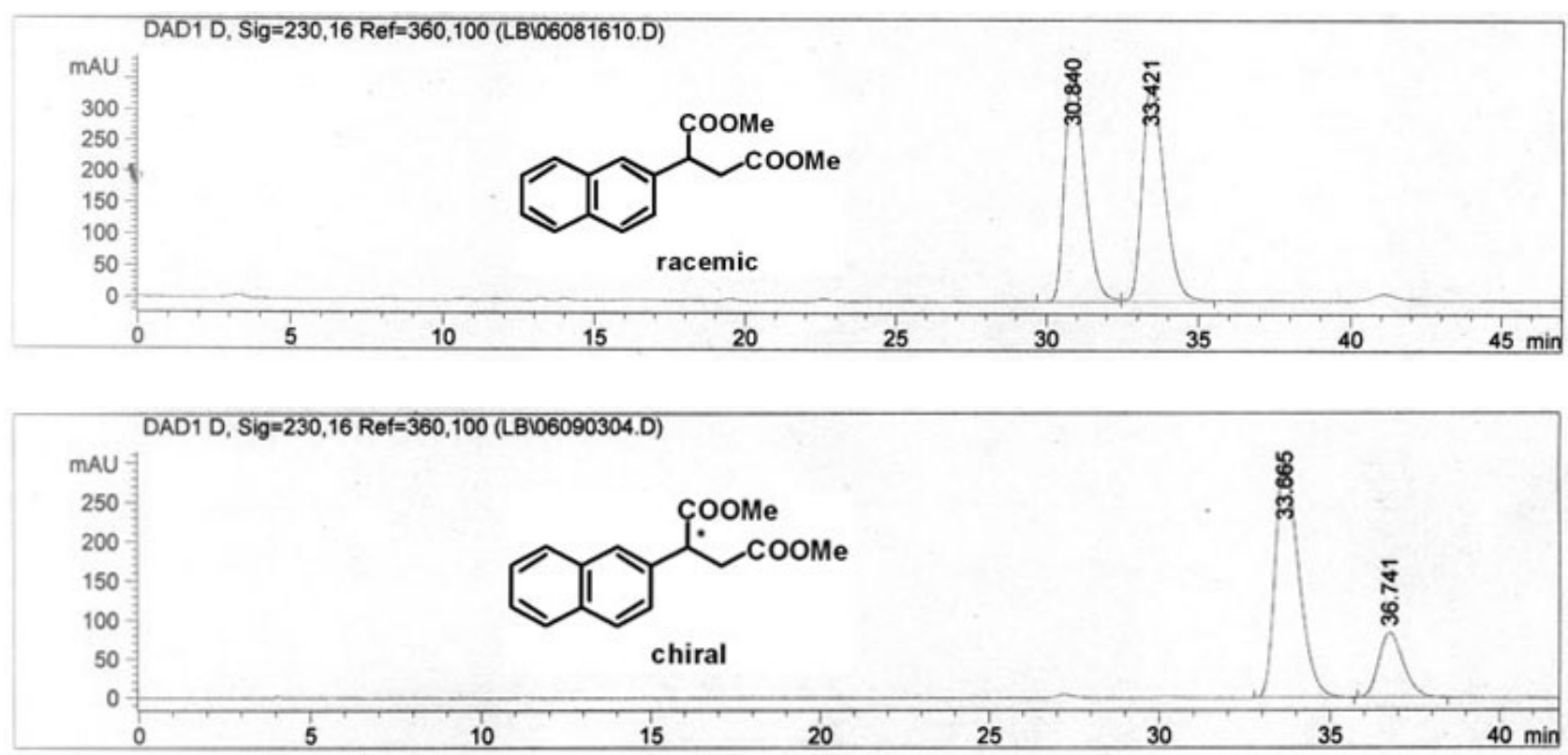

\section{Compound 27b (Table 4, entry 10)}

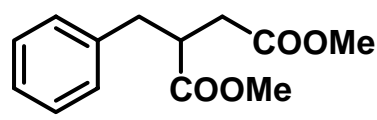

Product 27b was obtained in 91\% yield with $32 \%$ ee; ${ }^{1} \mathrm{H}$ NMR $\left(300 \mathrm{MHz}, \mathrm{CDCl}_{3}\right): \delta 2.37-2.44$ $(\mathrm{m}, 1 \mathrm{H}), 2.64-2.79(\mathrm{~m}, 2 \mathrm{H}), 3.03-3.17(\mathrm{~m}, 2 \mathrm{H}), 3.64(\mathrm{~s}, 3 \mathrm{H}), 3.67(\mathrm{~s}, 3 \mathrm{H}), 7.15-7.23(\mathrm{~m}, 2 \mathrm{H}), 7.25-7.32$ $(\mathrm{m}, 3 \mathrm{H}) ;{ }^{13} \mathrm{C}$ NMR $\left(75 \mathrm{MHz}, \mathrm{CDCl}_{3}\right): \delta 34.9,37.7,42.9,51.7,51.8,126.7,128.5,128.9,138.1,172.2$, 174.6; HRMS (EI): calcd for $\mathrm{C}_{13} \mathrm{H}_{16} \mathrm{O}_{4}\left(\mathrm{M}^{+}\right)$236.1049, found 236.1045; HPLC conditions: CHIRALCEL $\mathrm{OJ}-\mathrm{H}$, hexane $/ \mathrm{i}-\mathrm{PrOH}=90 / 10,0.8 \mathrm{~mL} / \mathrm{min}, 230 \mathrm{~nm}, 30^{\circ} \mathrm{C}, \mathrm{t}_{\mathrm{r}}($ major $)=15.7 \mathrm{~min}, \mathrm{t}_{\mathrm{r}}$ (minor) $=23.5 \mathrm{~min}$.

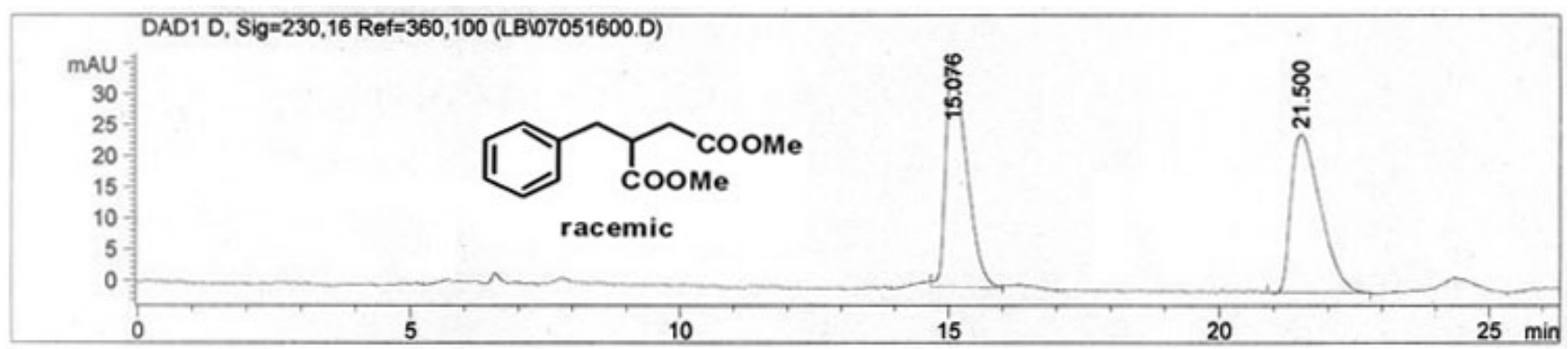




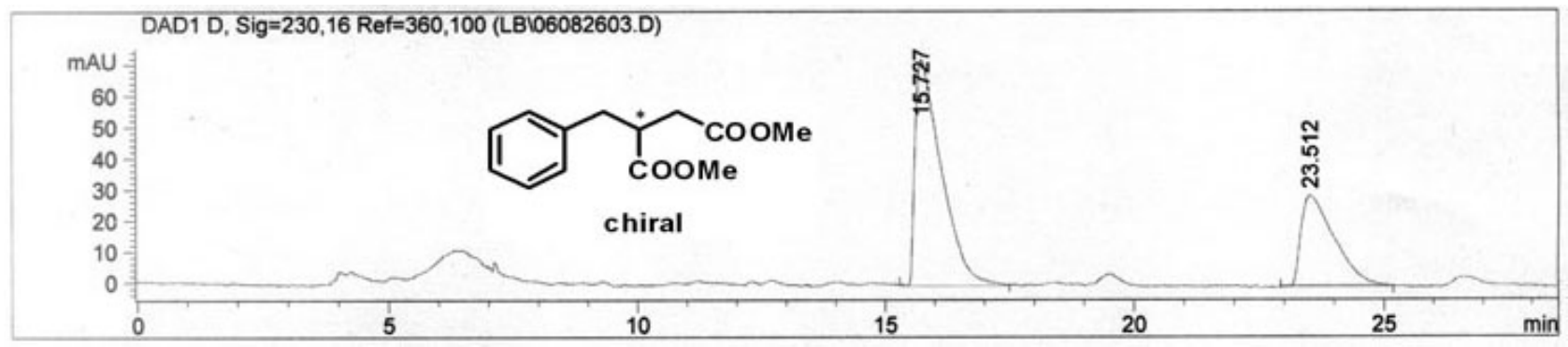

\section{Compound 28b (Table 5, entry 1)}<smiles>COC(=O)CC(C(C)=O)c1ccccc1</smiles>

Product 28b was obtained in 96\% yield with $57 \%$ ee; ${ }^{1} \mathrm{H}$ NMR $\left(300 \mathrm{MHz}, \mathrm{CDCl}_{3}\right): \delta 2.64-2.71(\mathrm{~m}$, $1 \mathrm{H}), 3.17-3.26(\mathrm{~m}, 1 \mathrm{H}), 3.64(\mathrm{~s}, 3 \mathrm{H}), 3.68(\mathrm{~s}, 3 \mathrm{H}), 4.08-4.13(\mathrm{~m}, 1 \mathrm{H}), 7.25-7.34(\mathrm{~m}, 5 \mathrm{H}) ;{ }^{13} \mathrm{C}$ NMR $(75$ $\mathrm{MHz}, \mathrm{CDCl}_{3}$ ): $\delta 37.5,46.9,51.8,52.3,127.6,127.6,128.8,137.6,171.9,173.3$; HRMS (EI): calcd for $\mathrm{C}_{12} \mathrm{H}_{14} \mathrm{O}_{4}\left(\mathrm{M}^{+}\right)$222.0892, found 222.0894; HPLC conditions: CHIRALCEL OJ-H, hexane/i-PrOH = $90 / 10,0.8 \mathrm{~mL} / \mathrm{min}, 230 \mathrm{~nm}, 30{ }^{\circ} \mathrm{C}, \mathrm{t}_{\mathrm{r}}($ minor $)=20.2 \mathrm{~min}, \mathrm{t}_{\mathrm{r}}$ (major) $=24.2 \mathrm{~min}$.
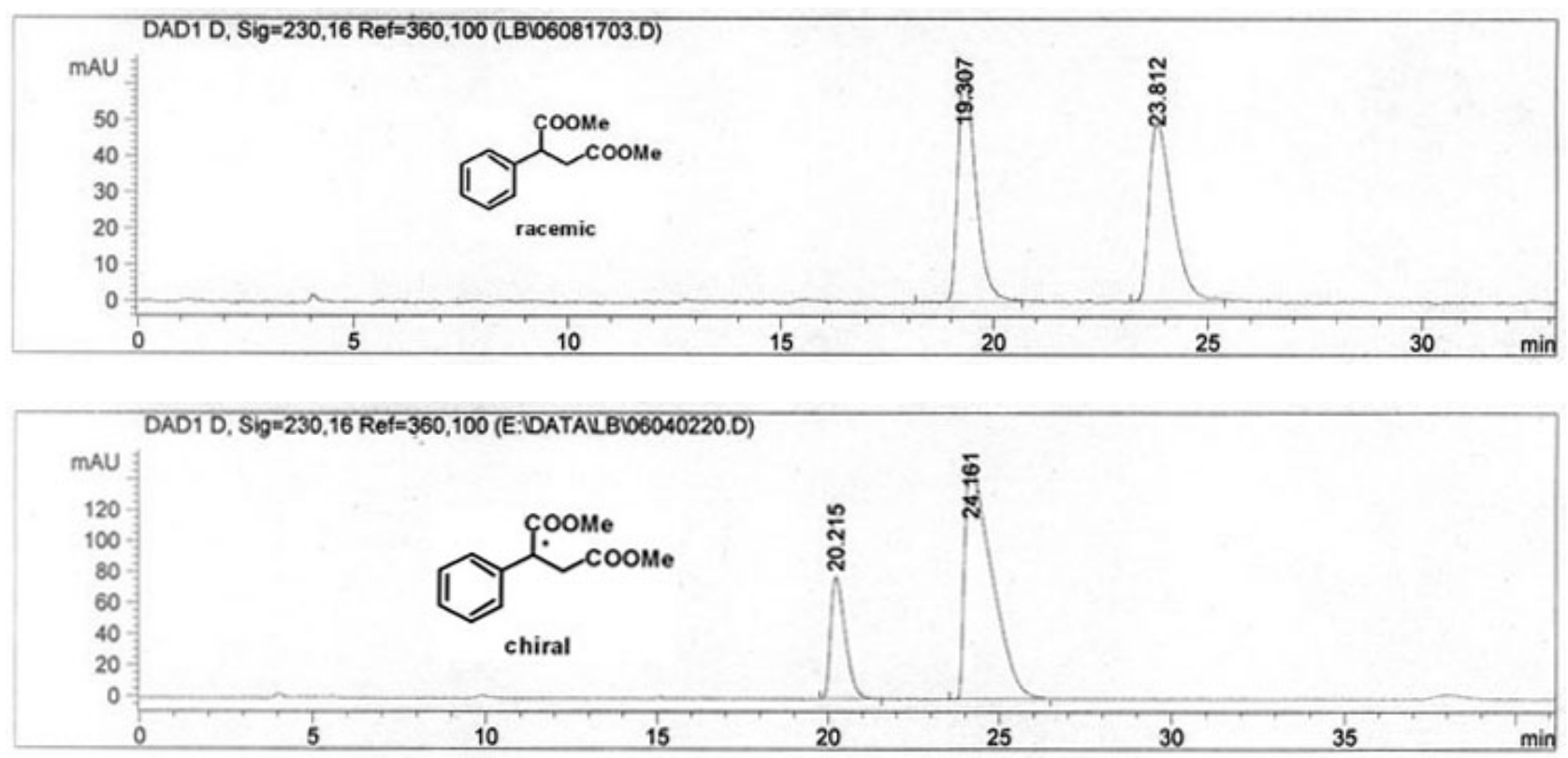

\section{Compound 29b (Table 5, entry 2)}


<smiles>COC(C)=O</smiles>

Product 29b was obtained in 90\% yield with $61 \% \mathrm{ee} ;{ }^{1} \mathrm{H}$ NMR $\left(300 \mathrm{MHz}, \mathrm{CDCl}_{3}\right): \delta 2.61-2.69(\mathrm{~m}$, $1 \mathrm{H}), 3.13-3.23(\mathrm{~m}, 1 \mathrm{H}), 3.67(\mathrm{~s}, 3 \mathrm{H}), 3.70(\mathrm{~s}, 3 \mathrm{H}), 3.81(\mathrm{~s}, 3 \mathrm{H}), 4.02-4.07(\mathrm{~m}, 1 \mathrm{H}), 6.85-6.89(\mathrm{~m}, 2 \mathrm{H})$, 7.19-7.22 (m, 2H); ${ }^{13} \mathrm{C}$ NMR (75 MHz, $\left.\mathrm{CDCl}_{3}\right): \delta 37.6,46.1,51.7,52.2,55.1,114.1,128.7,129.6,158.9$, 171.9, 173.6; HRMS (EI): calcd for $\mathrm{C}_{13} \mathrm{H}_{16} \mathrm{O}_{5}\left(\mathrm{M}^{+}\right)$252.0998, found 252.1000; HPLC conditions: CHIRALCEL OJ-H, hexane $/ \mathrm{i}-\mathrm{PrOH}=90 / 10,0.8 \mathrm{~mL} / \mathrm{min}, 230 \mathrm{~nm}, 30{ }^{\circ} \mathrm{C}, \mathrm{t}_{\mathrm{r}}$ (major) $=28.1 \mathrm{~min}, \mathrm{t}_{\mathrm{r}}$ (minor) $=31.4 \mathrm{~min}$.
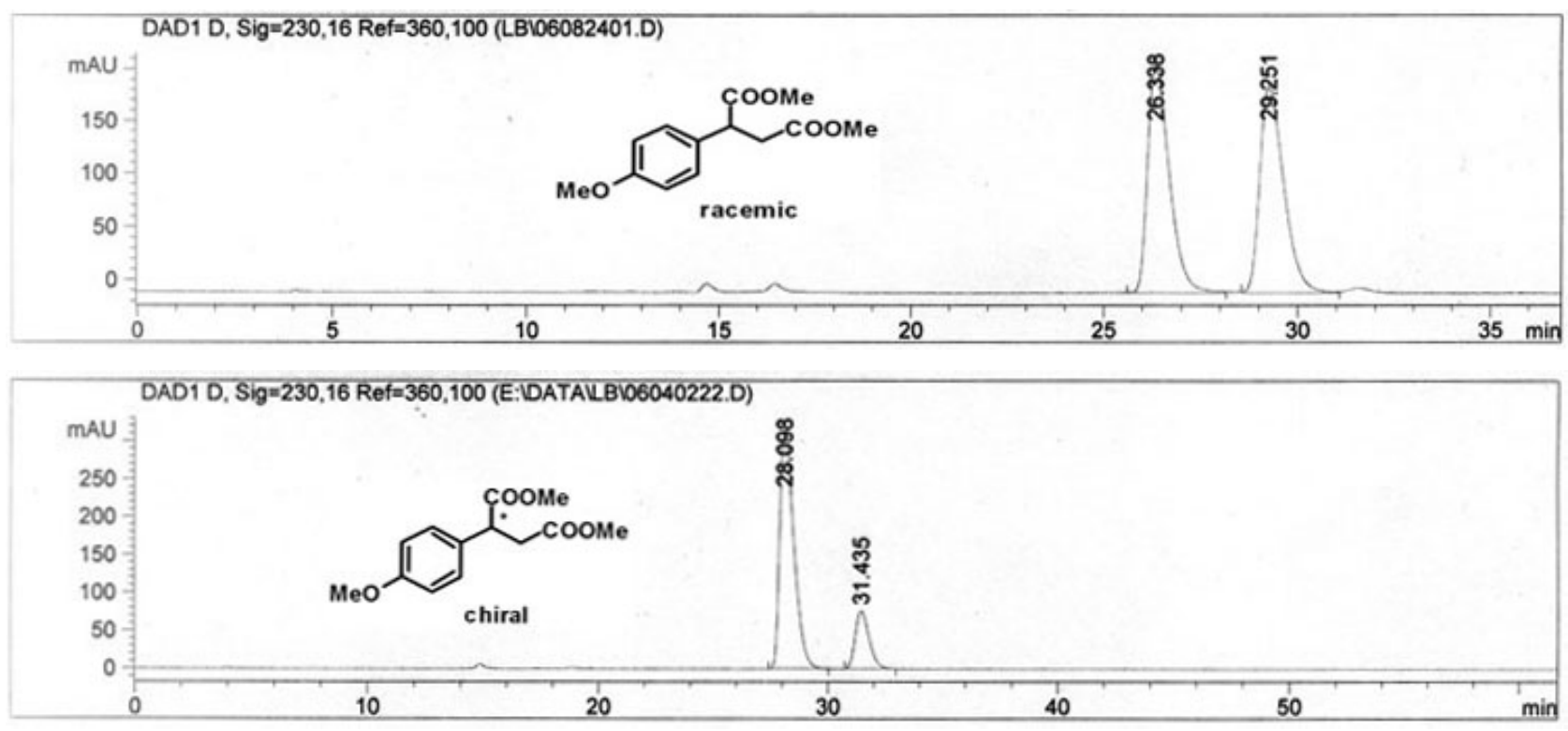

\section{Compound 30b (Table 5, entry 3)}<smiles>COC(C)=O</smiles>

Product 30b was obtained in 96\% yield with $61 \%$ ee; ${ }^{1} \mathrm{H}$ NMR $\left(300 \mathrm{MHz}, \mathrm{CDCl}_{3}\right): \delta 2.32(\mathrm{~s}, 3 \mathrm{H})$, 2.61-2.68 (m, 1H), 3.15-3.24 (m, 1H), $3.67(\mathrm{~s}, 6 \mathrm{H}), 4.04-4.09(\mathrm{~m}, 1 \mathrm{H}), 7.12-7.19(\mathrm{~m}, 4 \mathrm{H}) ;{ }^{13} \mathrm{C}$ NMR $(75$ $\mathrm{MHz}, \mathrm{CDCl}_{3}$ ): $\delta 20.8,37.4,46.5,51.6,52.1,127.4,129.4,134.5,137.2,171.8,173.4$; HRMS (EI): calcd for $\mathrm{C}_{13} \mathrm{H}_{16} \mathrm{O}_{4}\left(\mathrm{M}^{+}\right)$236.1049, found 236.1047; HPLC conditions: CHIRALCEL OJ-H, hexane/i-PrOH = $90 / 10,0.8 \mathrm{~mL} / \mathrm{min}, 230 \mathrm{~nm}, 30{ }^{\circ} \mathrm{C}, \mathrm{t}_{\mathrm{r}}($ major $)=14.4 \mathrm{~min}, \mathrm{t}_{\mathrm{r}}($ minor $)=18.7 \mathrm{~min}$. 

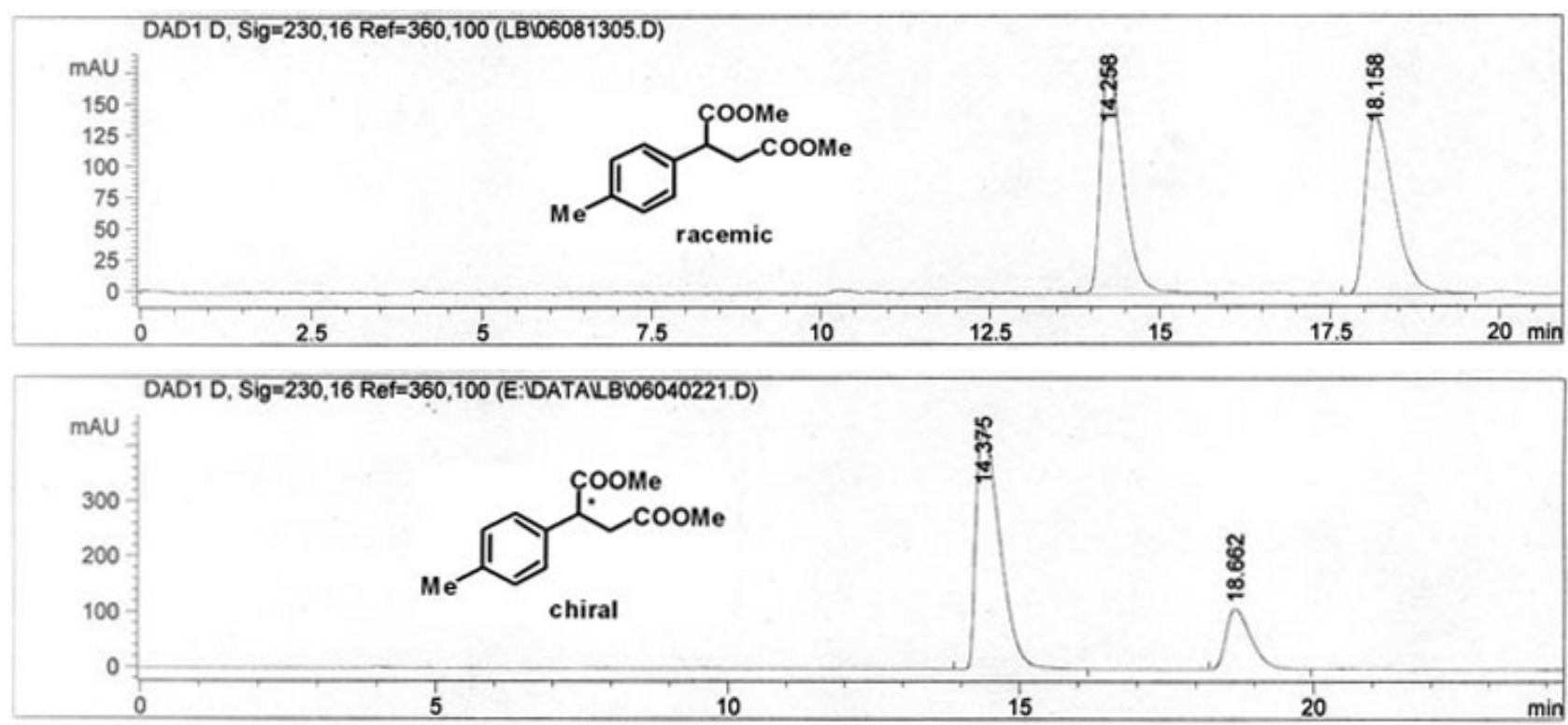

\section{Compound 31b (Table 5, entry 4)}<smiles>COC(=O)CC(C(C)=O)c1ccc(Cl)cc1</smiles>

Product 31b was obtained in 95\% yield with 55\% ee; ${ }^{1} \mathrm{H}$ NMR $\left(300 \mathrm{MHz}, \mathrm{CDCl}_{3}\right): \delta 2.63-2.70(\mathrm{~m}$, $1 \mathrm{H}), 3.14-3.23(\mathrm{~m}, 1 \mathrm{H}), 3.67(\mathrm{~s}, 3 \mathrm{H}), 3.68(\mathrm{~s}, 3 \mathrm{H}), 4.05-4.10(\mathrm{~m}, 1 \mathrm{H}), 7.22-7.25(\mathrm{~m}, 2 \mathrm{H}), 7.29-7.32(\mathrm{~m}$, $2 \mathrm{H}) ;{ }^{13} \mathrm{C}$ NMR $\left(75 \mathrm{MHz}, \mathrm{CDCl}_{3}\right): \delta 37.3,46.4,51.9,52.4,128.9,129.1,133.6,136.0,171.6,172.9$; HRMS (EI): calcd for $\mathrm{C}_{12} \mathrm{H}_{13} \mathrm{O}_{4}{ }^{35} \mathrm{Cl}\left(\mathrm{M}^{+}\right)$256.0502, found 256.0503; HPLC conditions: CHIRALCEL $\mathrm{OJ}-\mathrm{H}$, hexane $/ \mathrm{i}-\mathrm{PrOH}=90 / 10,0.8 \mathrm{~mL} / \mathrm{min}, 230 \mathrm{~nm}, 30{ }^{\circ} \mathrm{C}$, $\mathrm{t}_{\mathrm{r}}$ (major) $=13.1 \mathrm{~min}, \mathrm{t}_{\mathrm{r}}($ minor $)=14.5 \mathrm{~min}$.

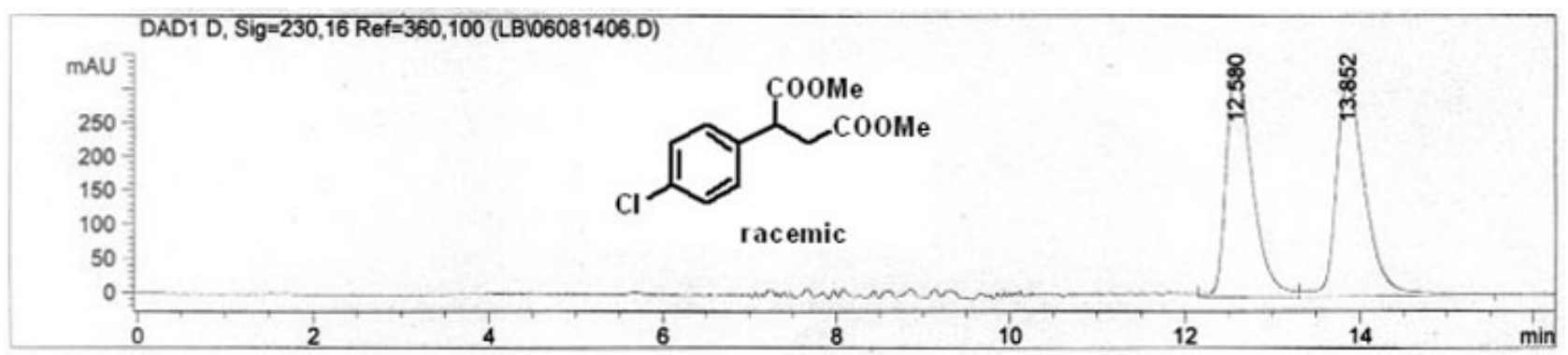




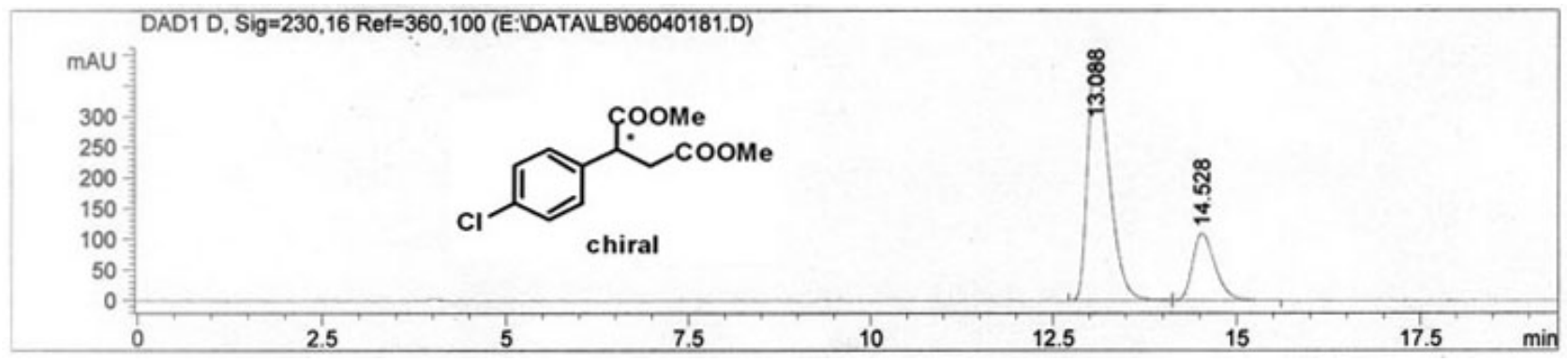




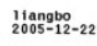

$\mid$
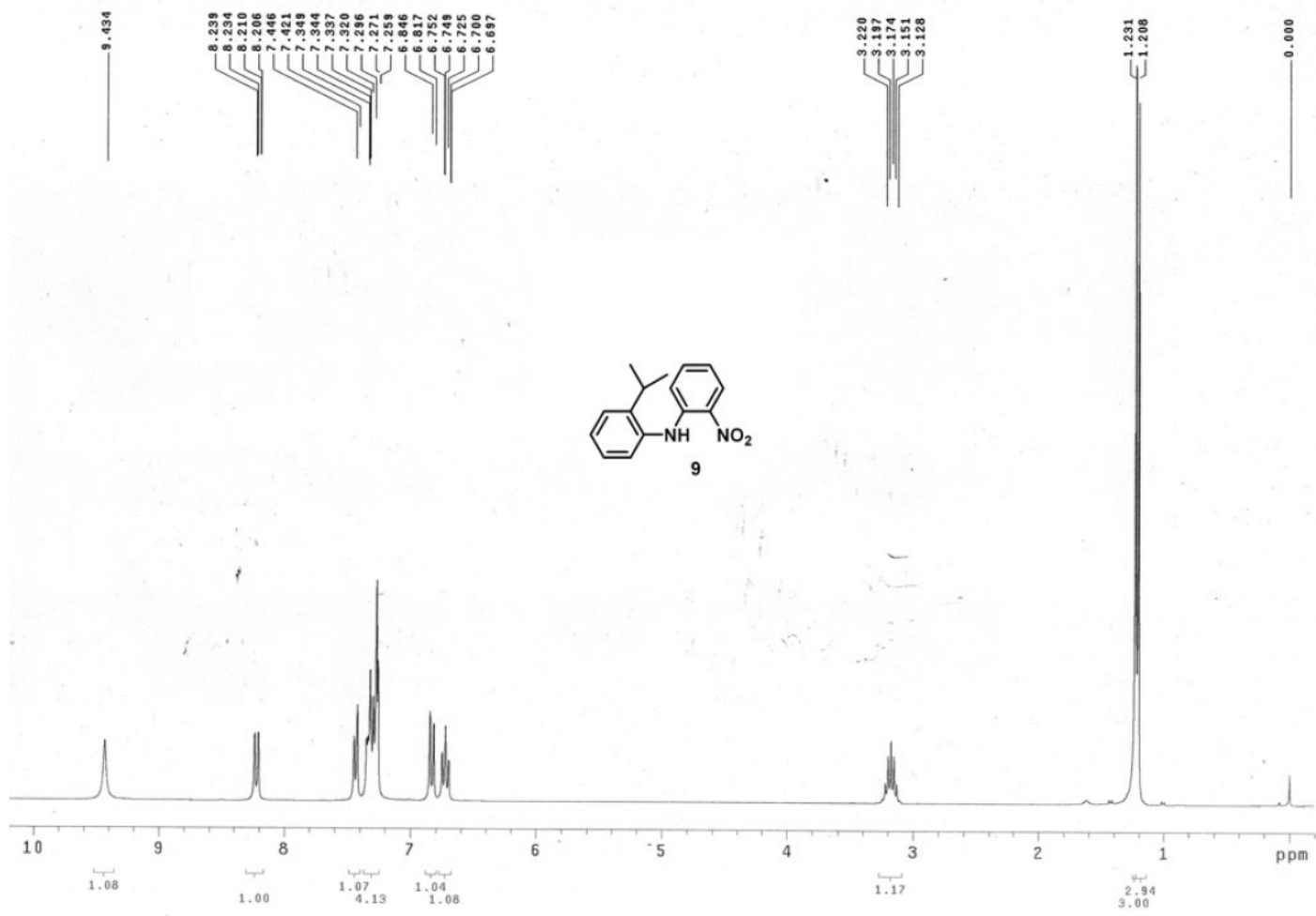

11:angbo

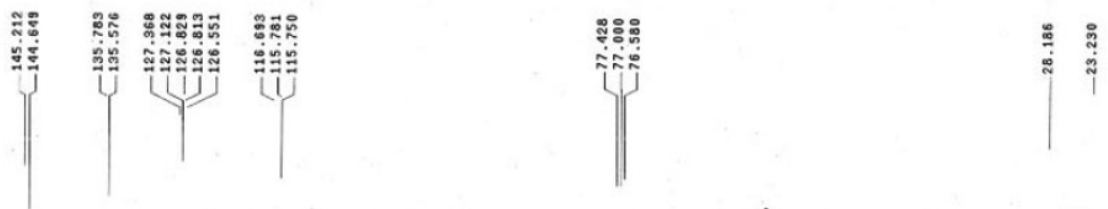

-

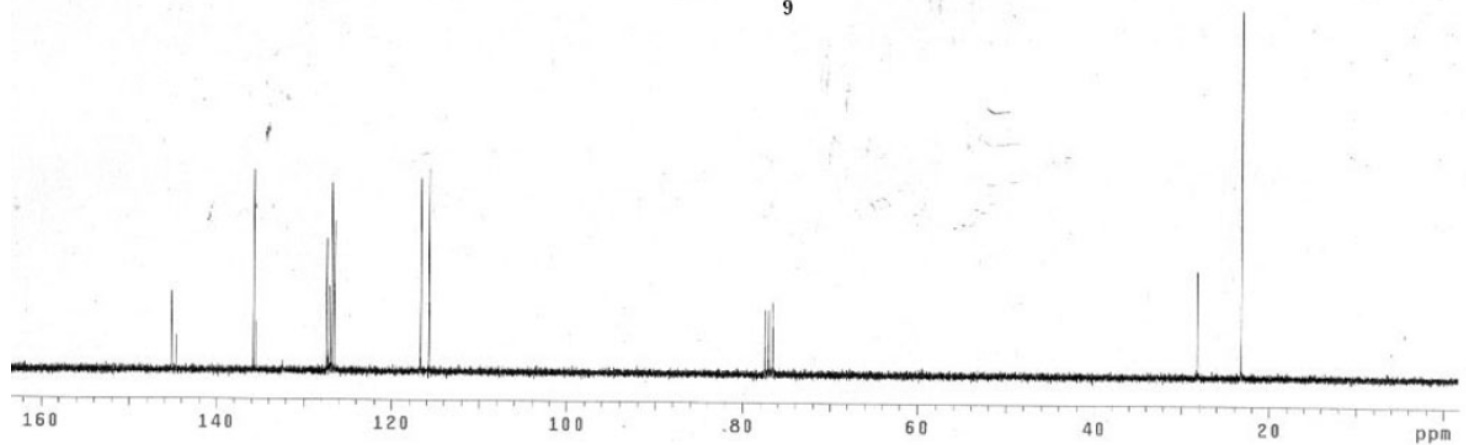


111angopo
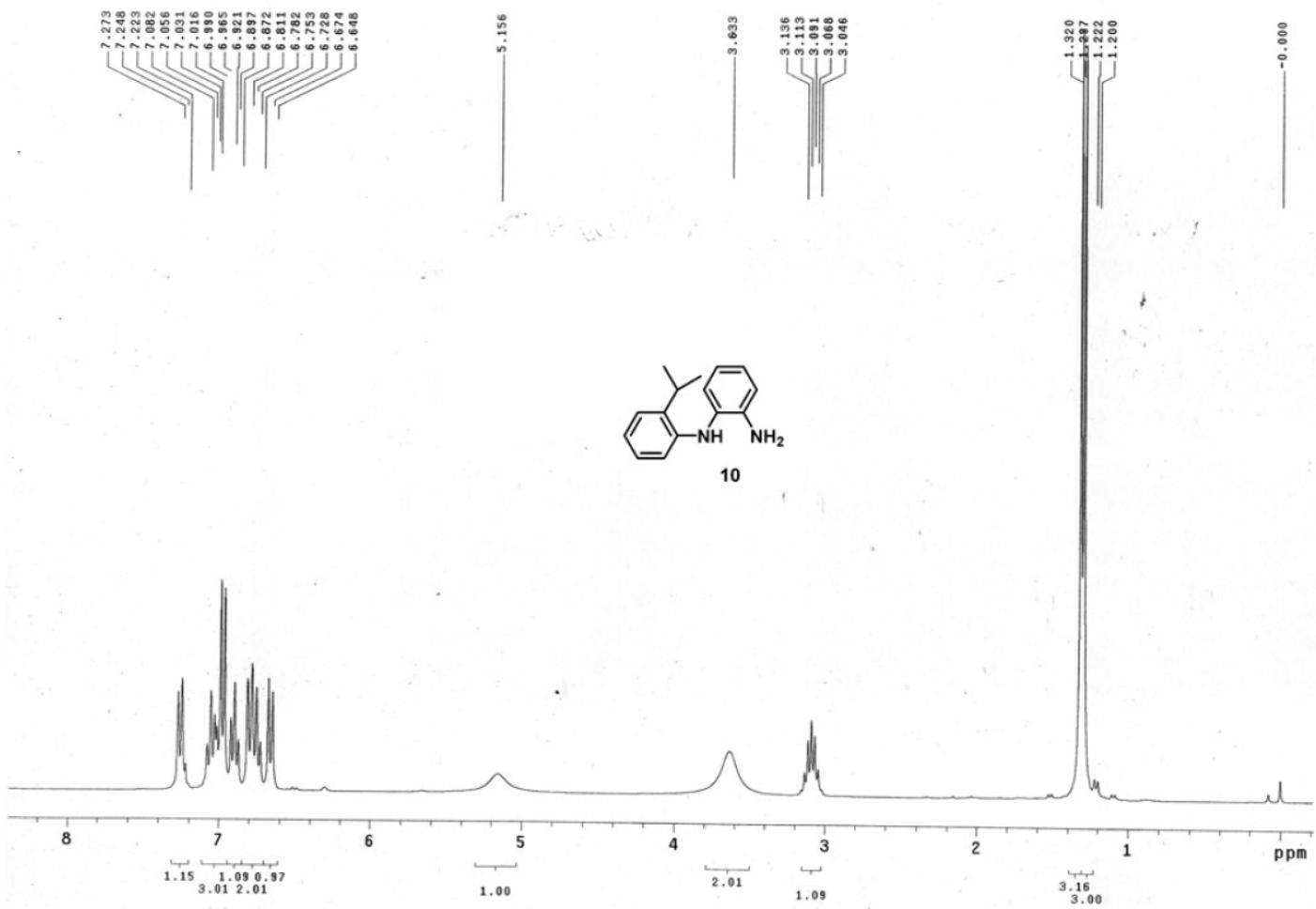

11tangbo
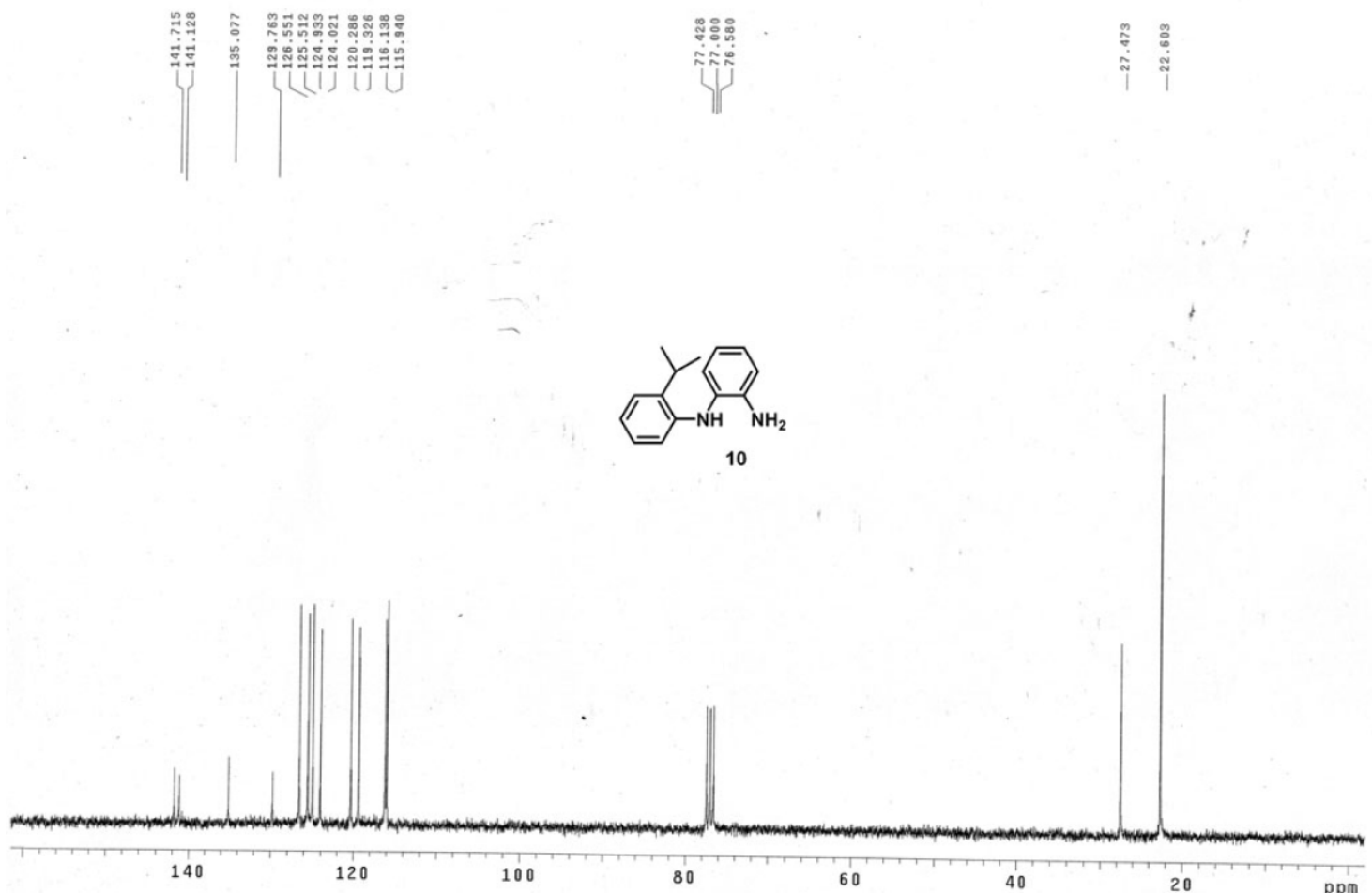


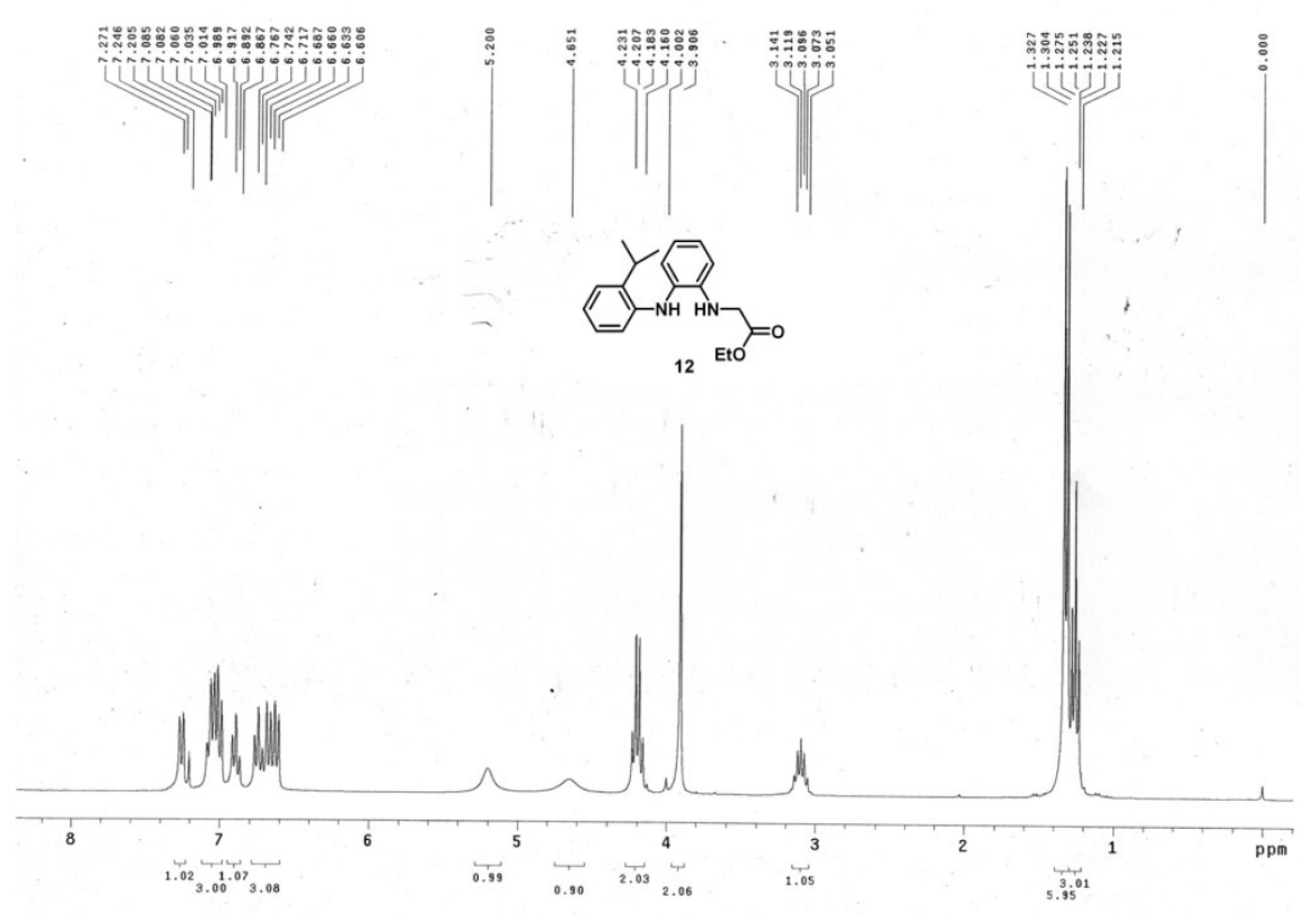

111ango
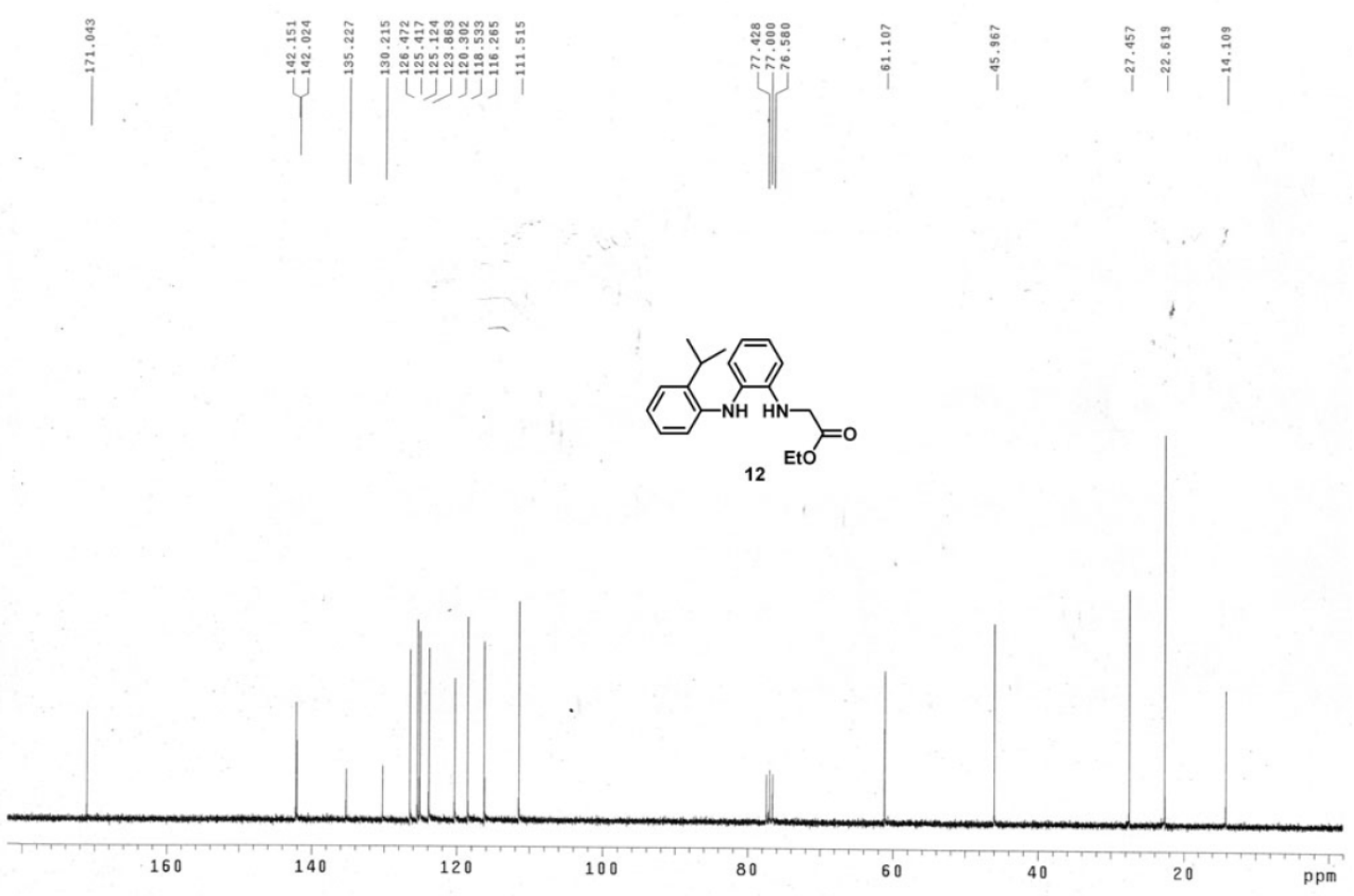
1soopropyl-thiourea
$2005-12-27$

|
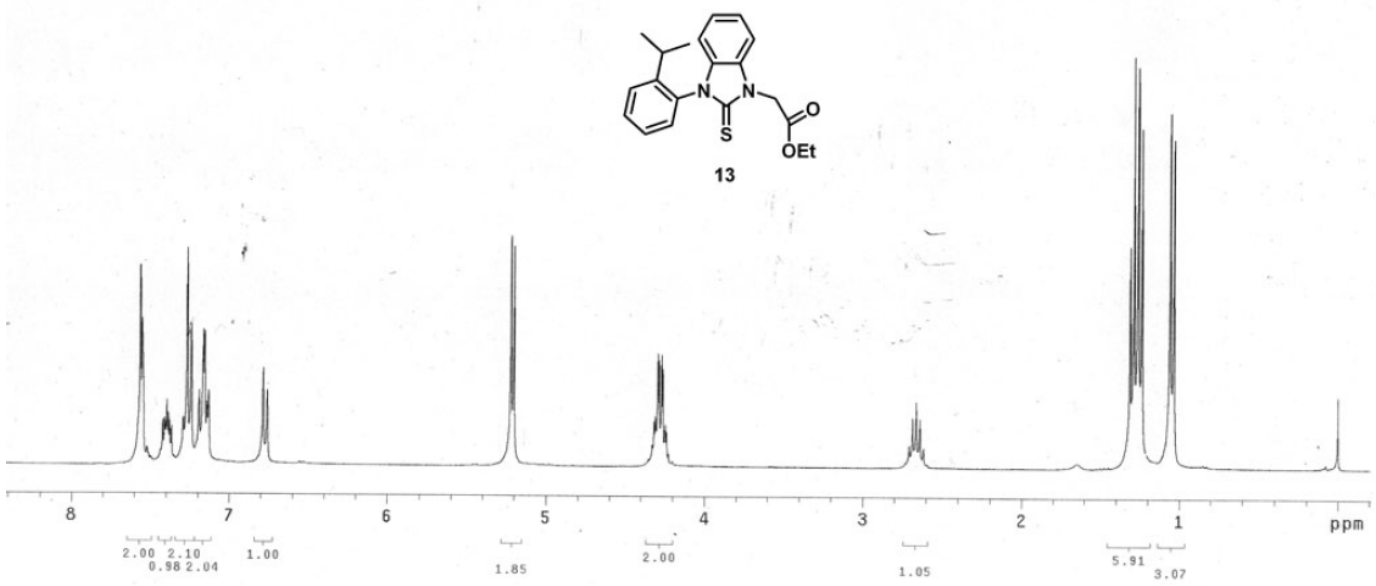

is sopropyl-thiourea
$2005-12-27$
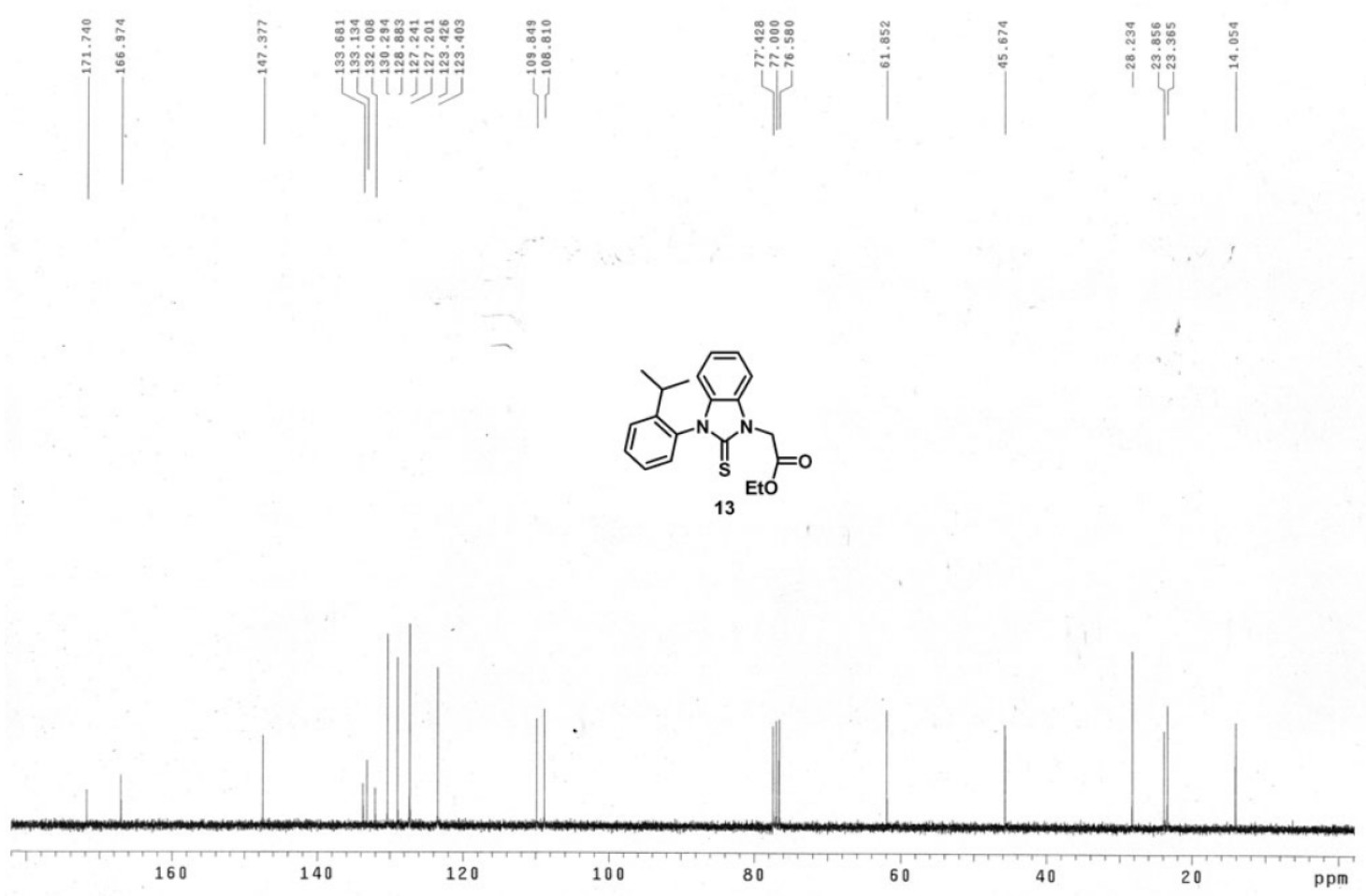
$\frac{14041 n 9}{2006-1-6}$

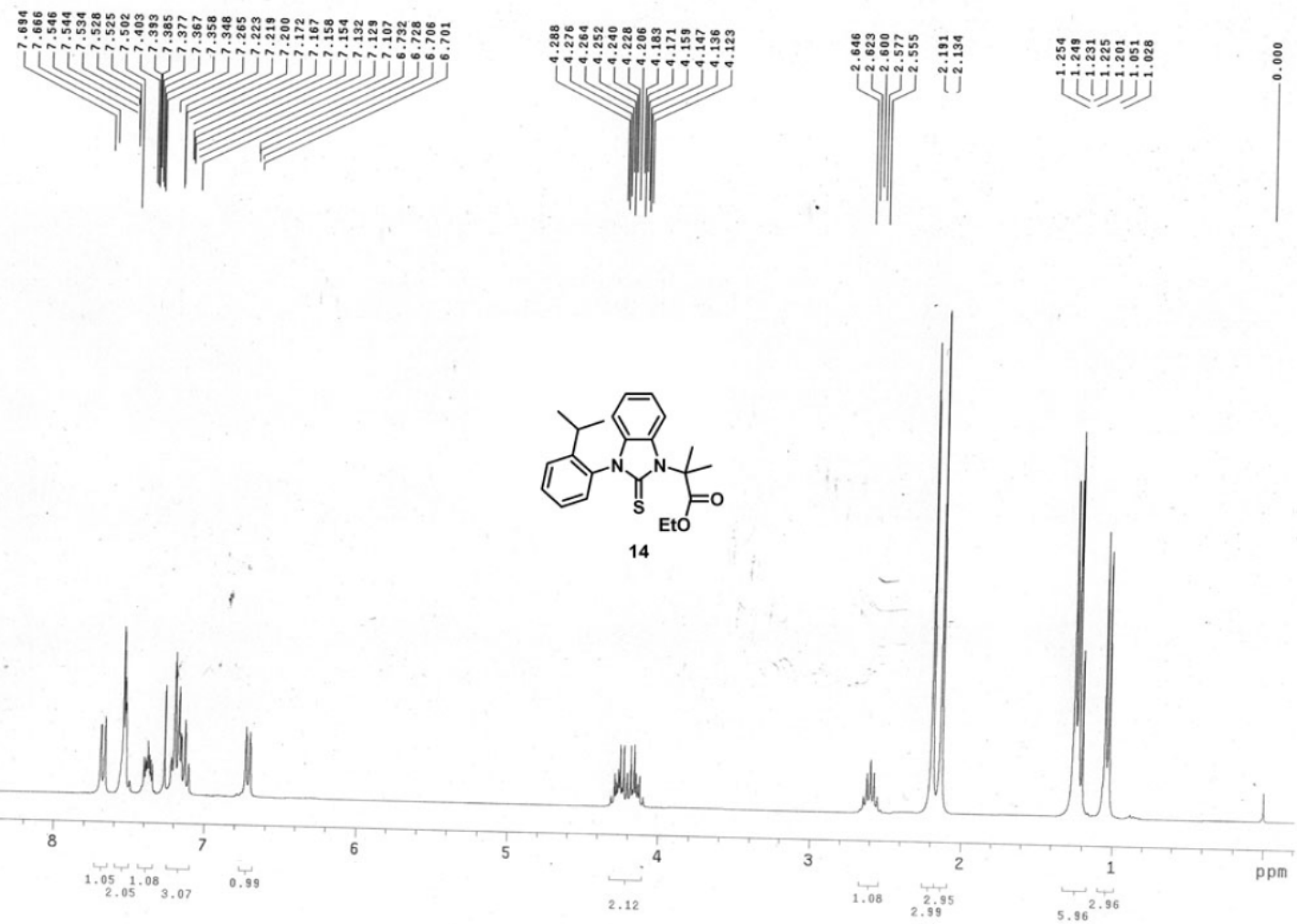

2006-1-6

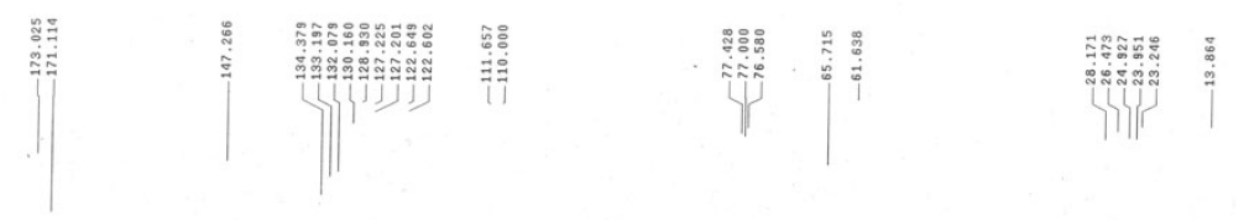
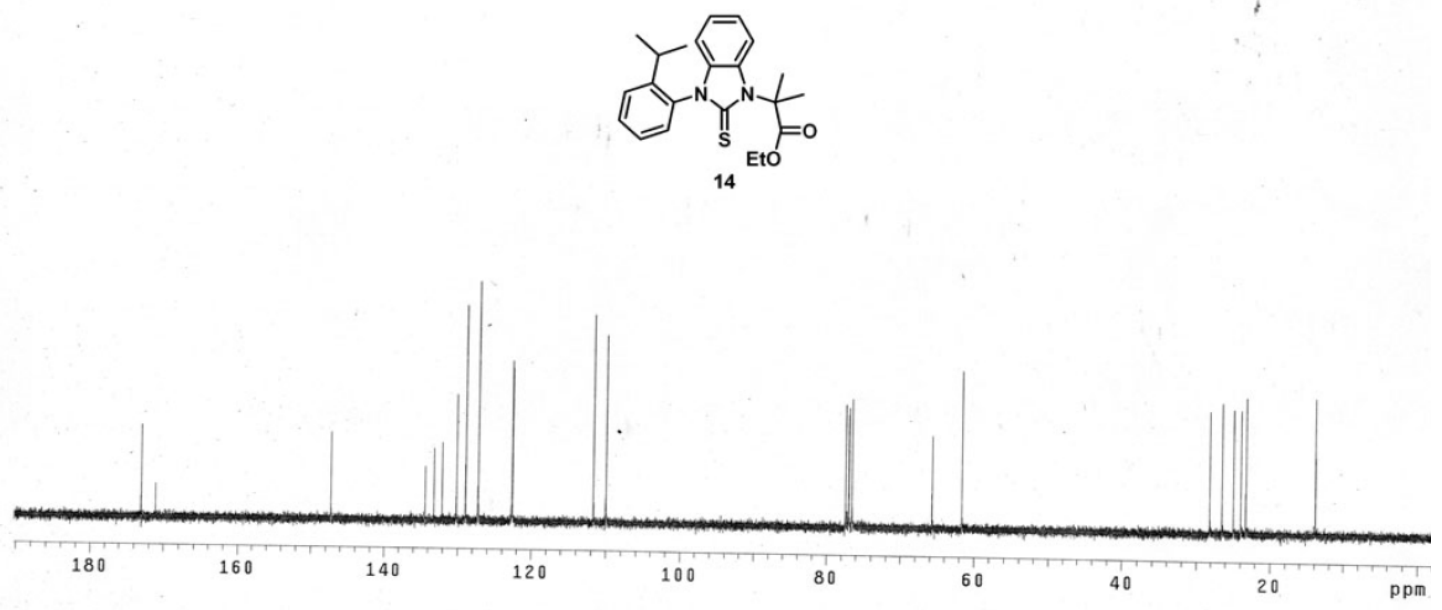
ipr 2 2me thiourea cooH
$300 \mathrm{~m}$

Archive directory: /export/home/vnmr1/vnmrsys/data
Sample directory:
Fillo

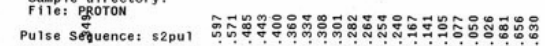

iniiuiuijijijijijij

||
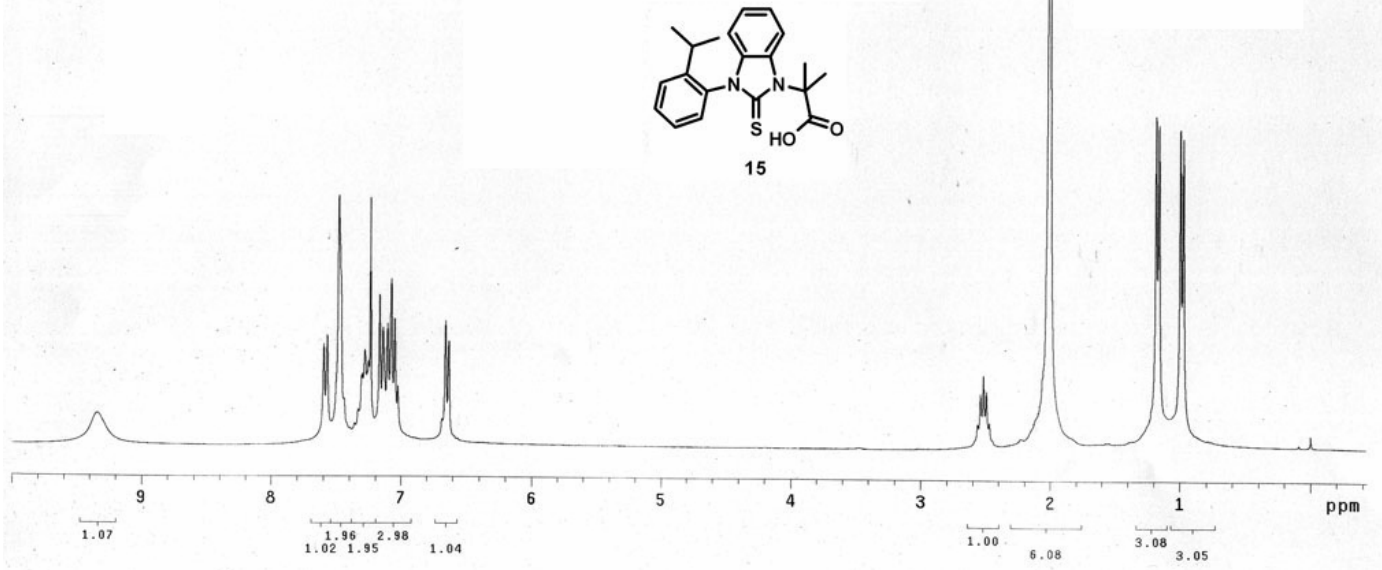

$\mathrm{C}_{33-13} \mathrm{pr}$ 2Me thiourea
$300 \mathrm{MO}$

Pulse Sequence: $52 \mathrm{p}$
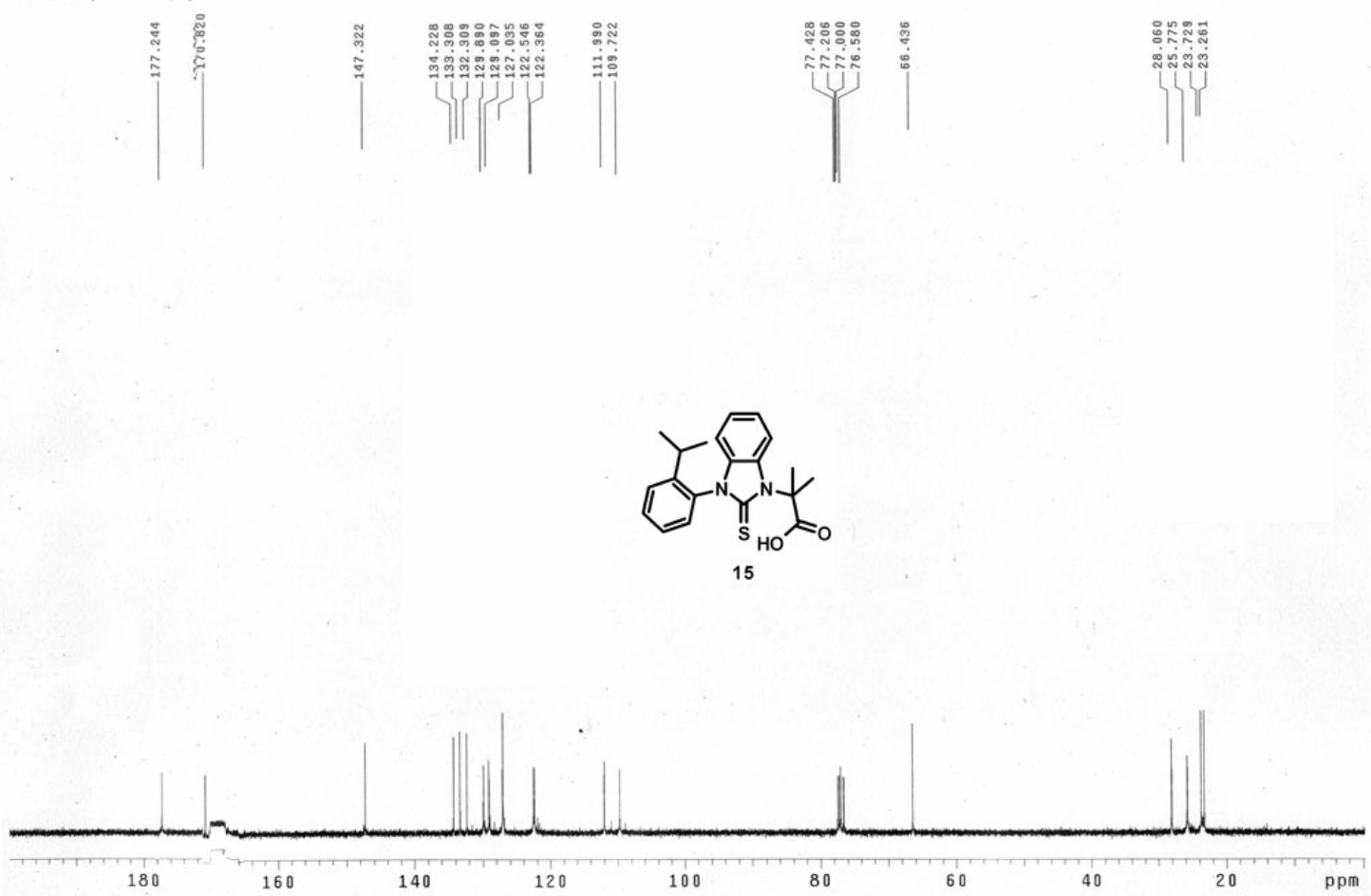


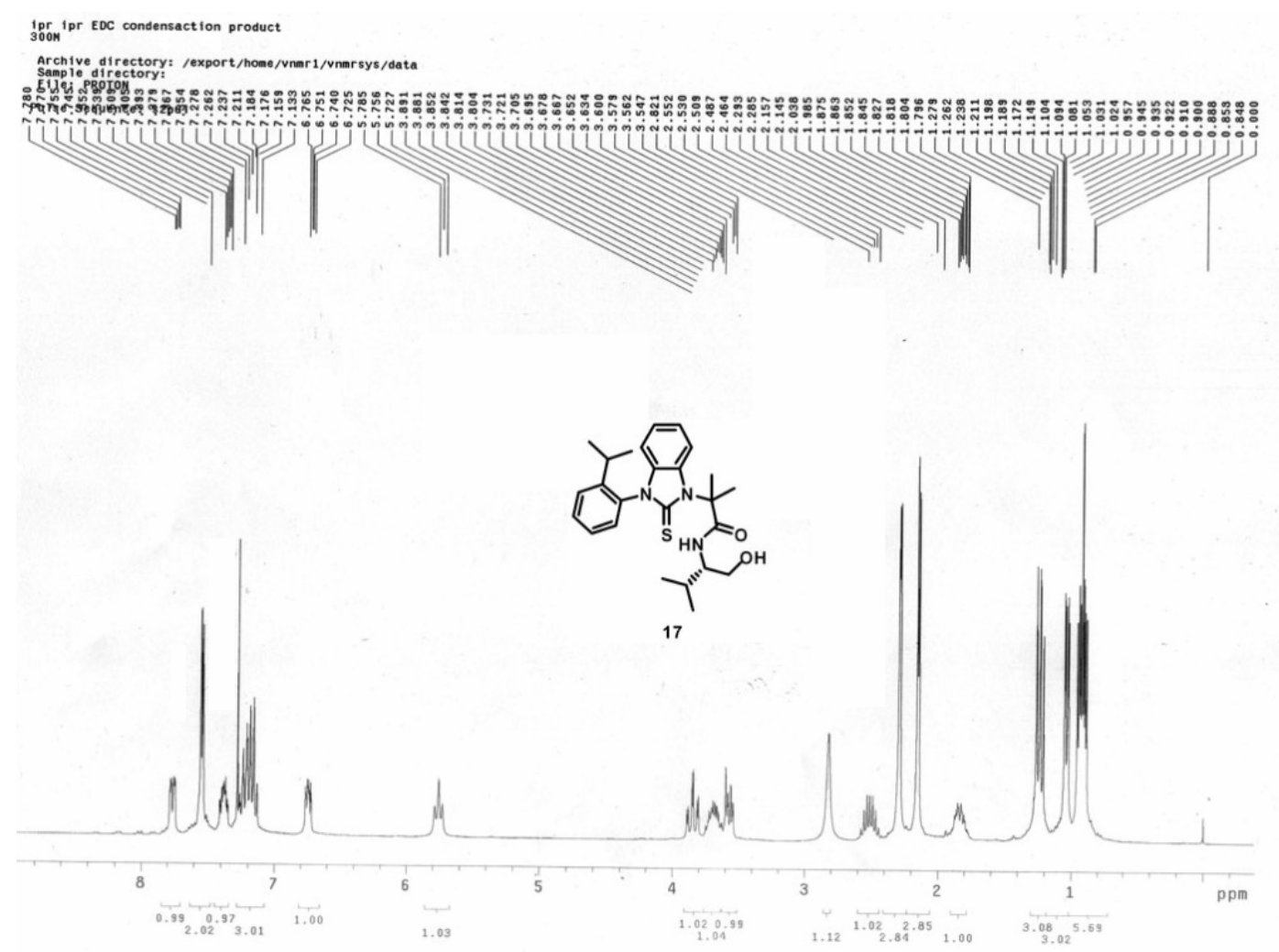

C13-1pr Ipr EDC condensaction product
$300 \mathrm{M}$

Pulse Sequence: s2pul
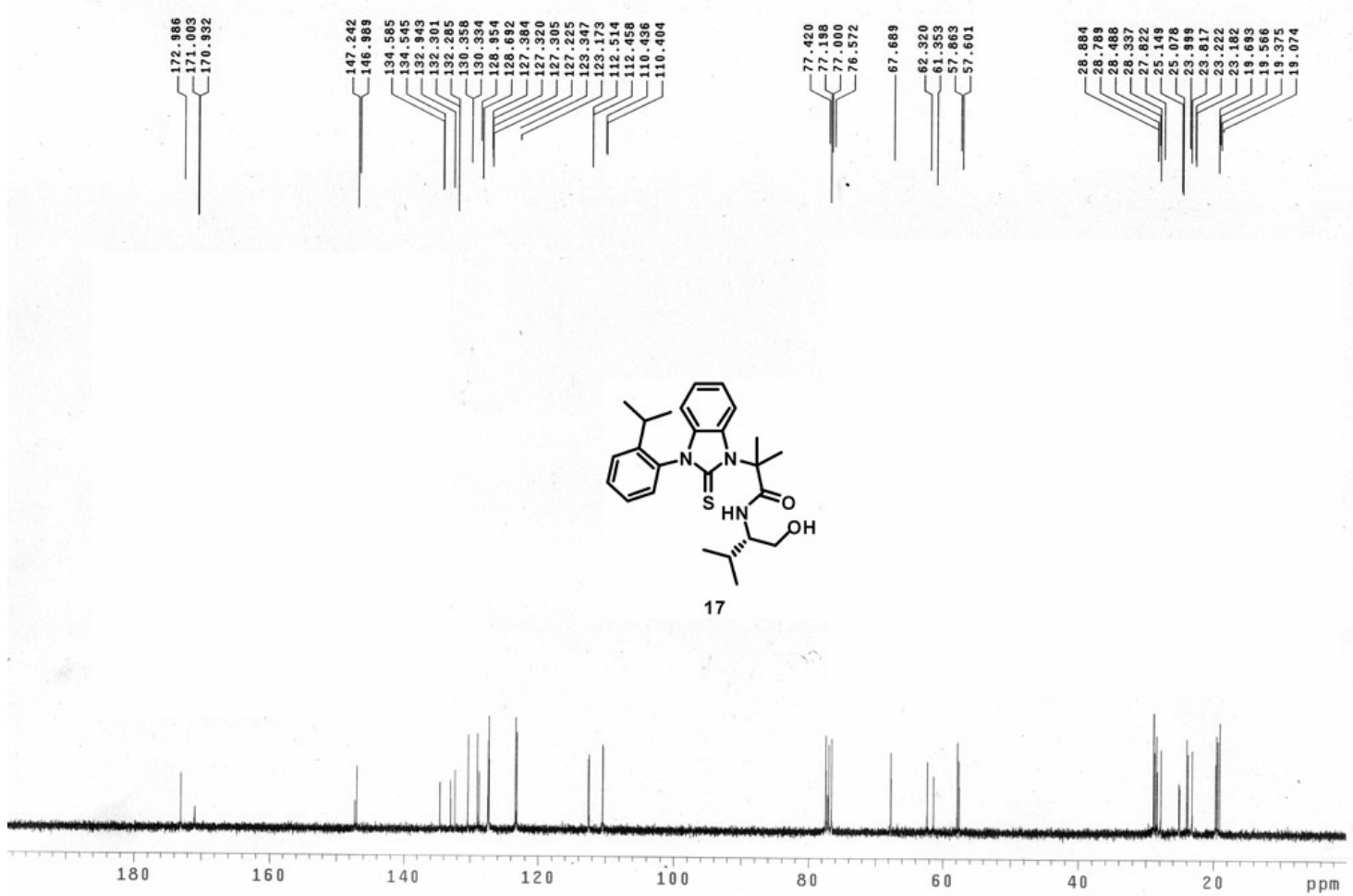


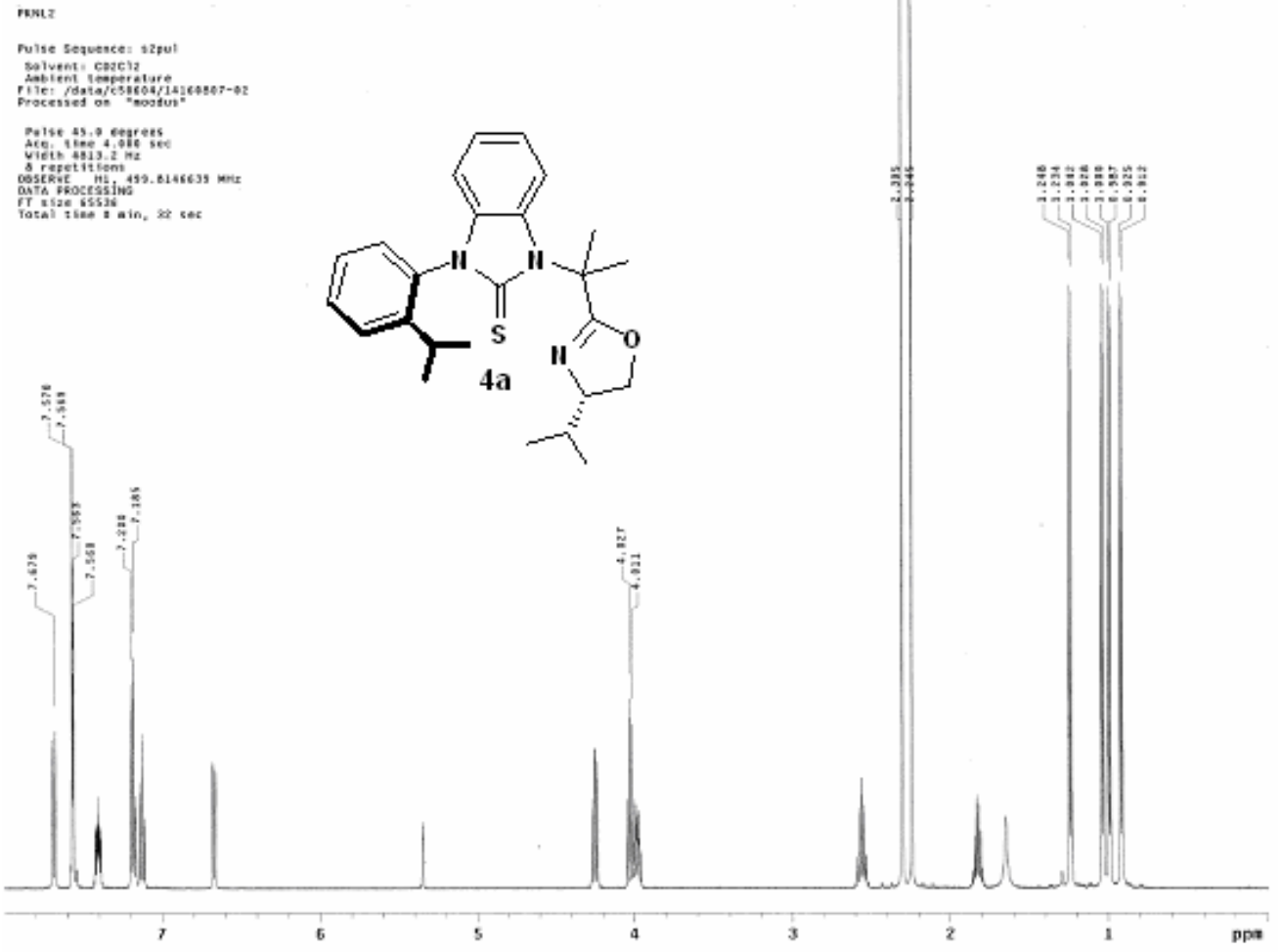


meas

cerse setuences stpat

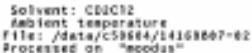

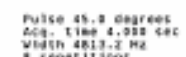

of

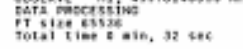

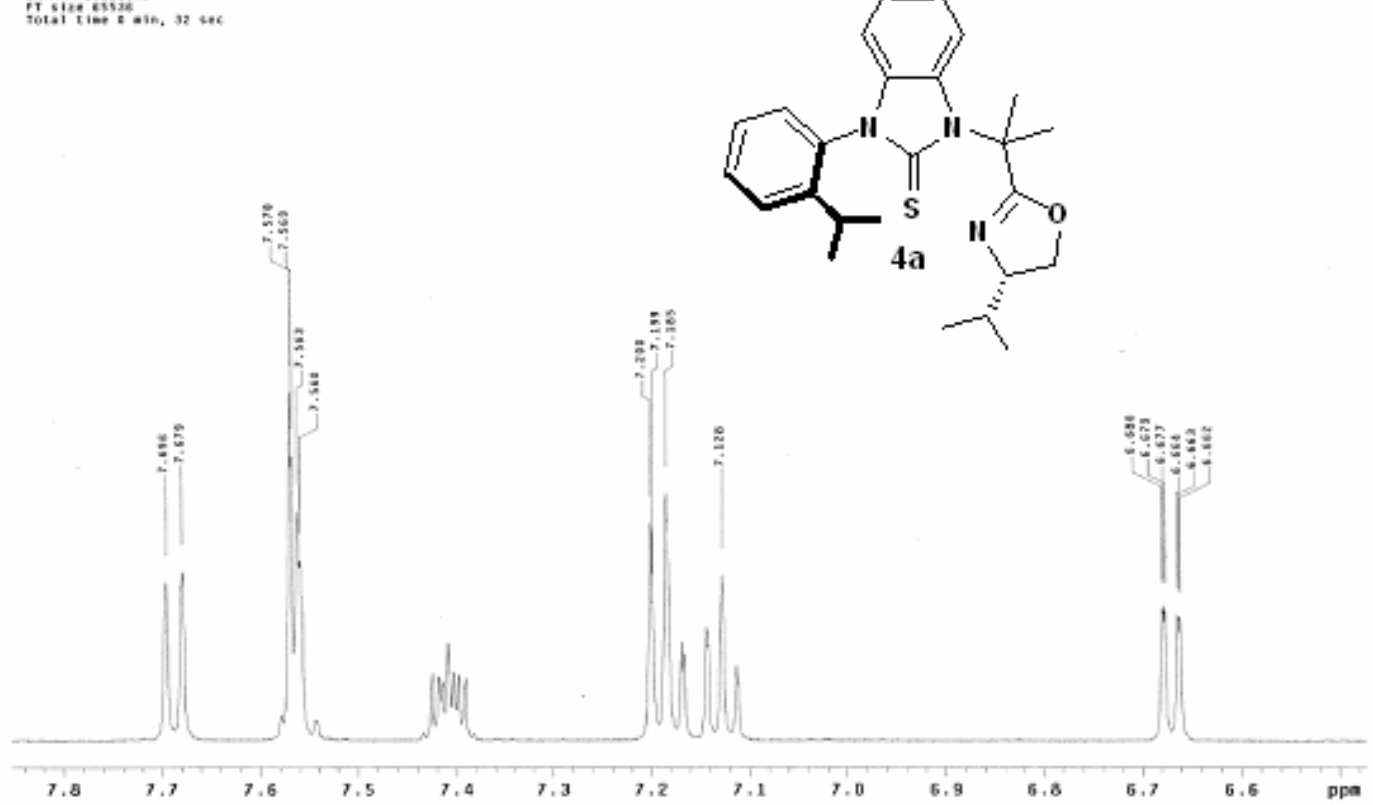

sect:

Pulse Eecuenter sapu

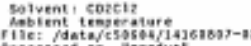

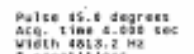

oosteptitions

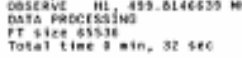

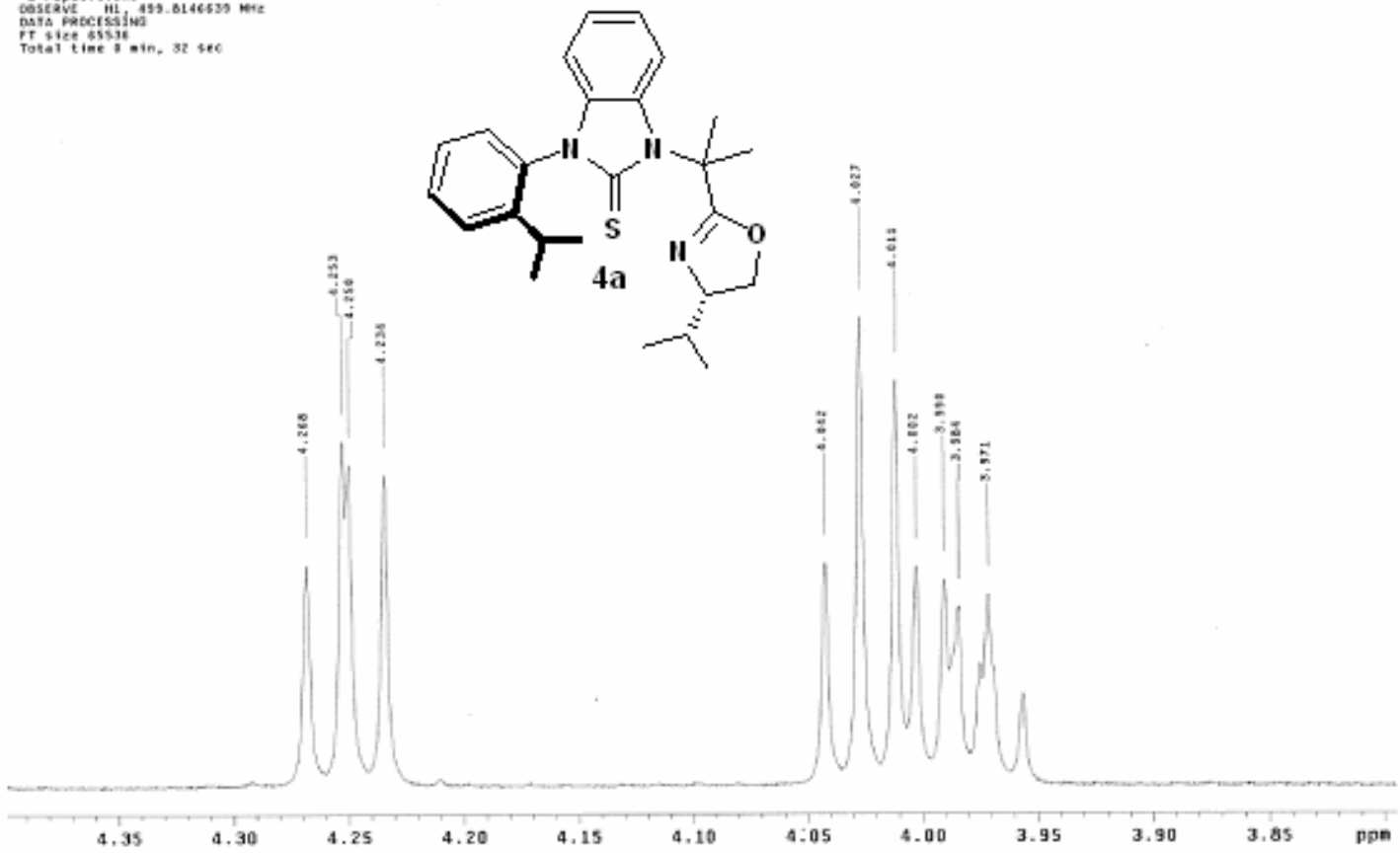




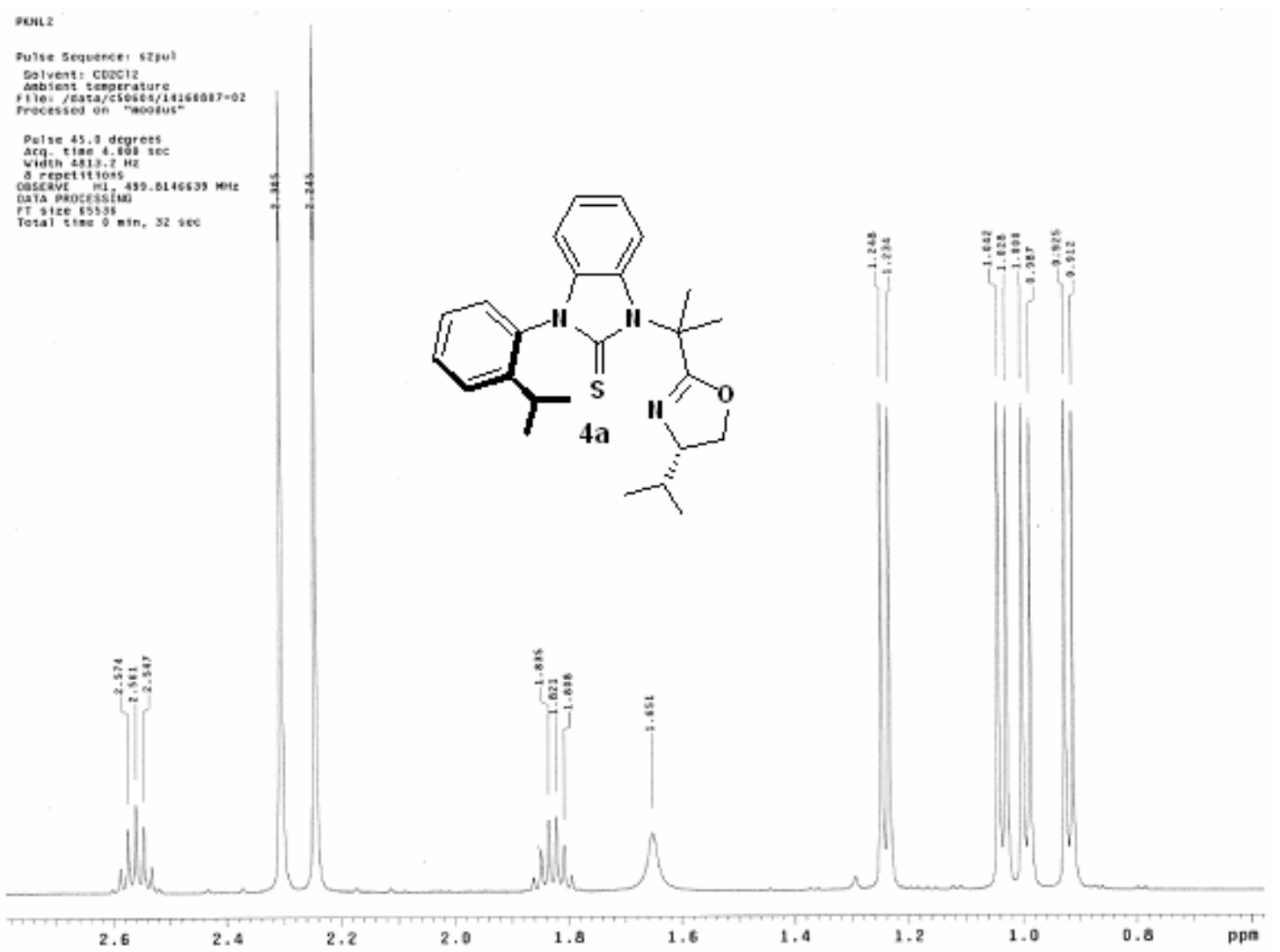


next:

pust sequesces stput

Solvem: cascla

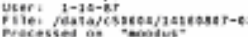

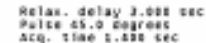

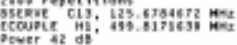

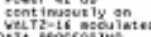

tise

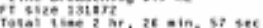
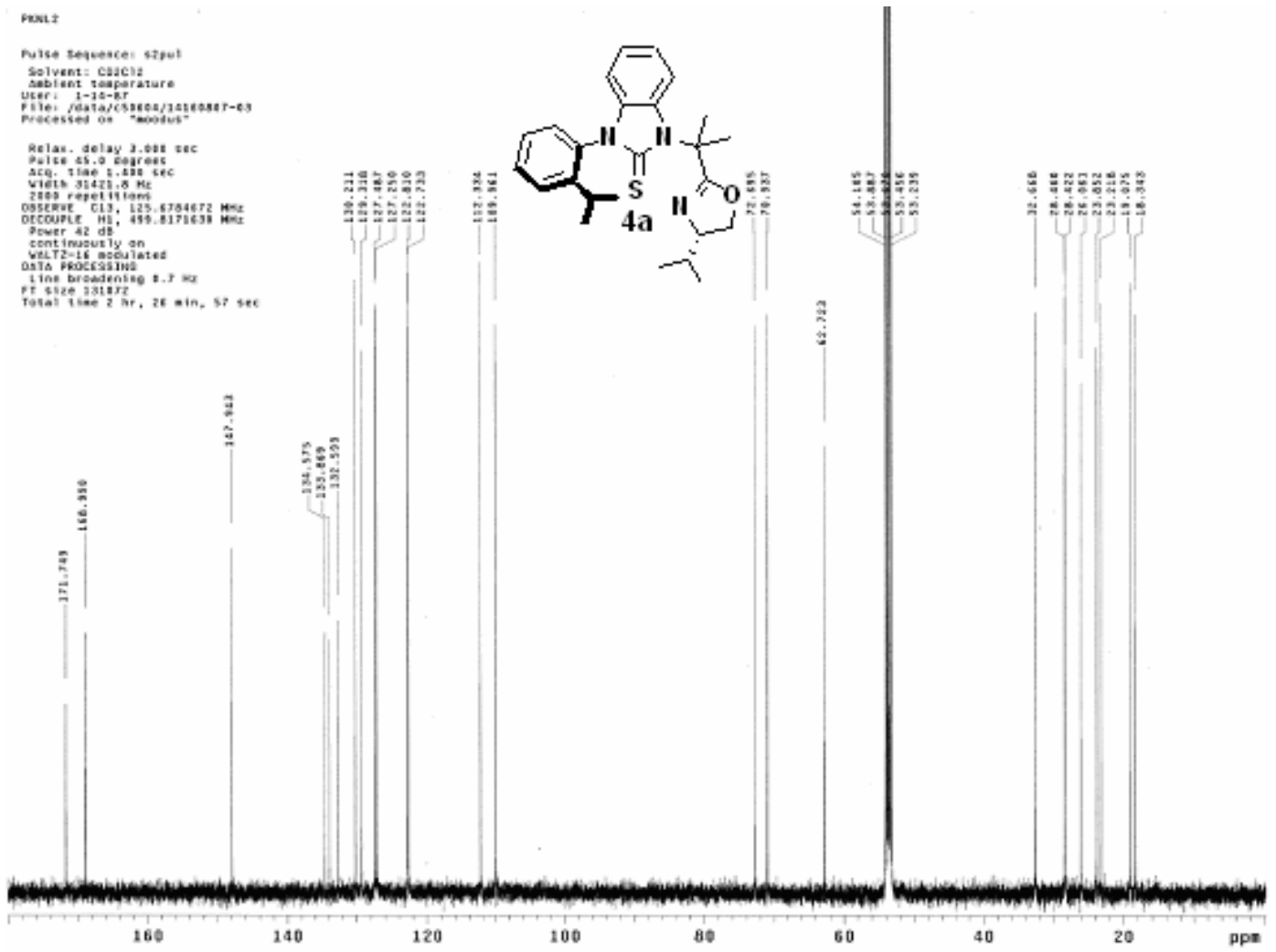

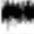

กองเ2

180

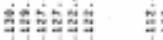

Fulve sequente: szpu

Solren: cozenz

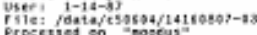

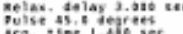

xyin

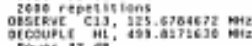

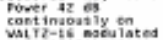

tine brodseniting 0.7 me

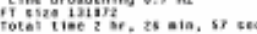
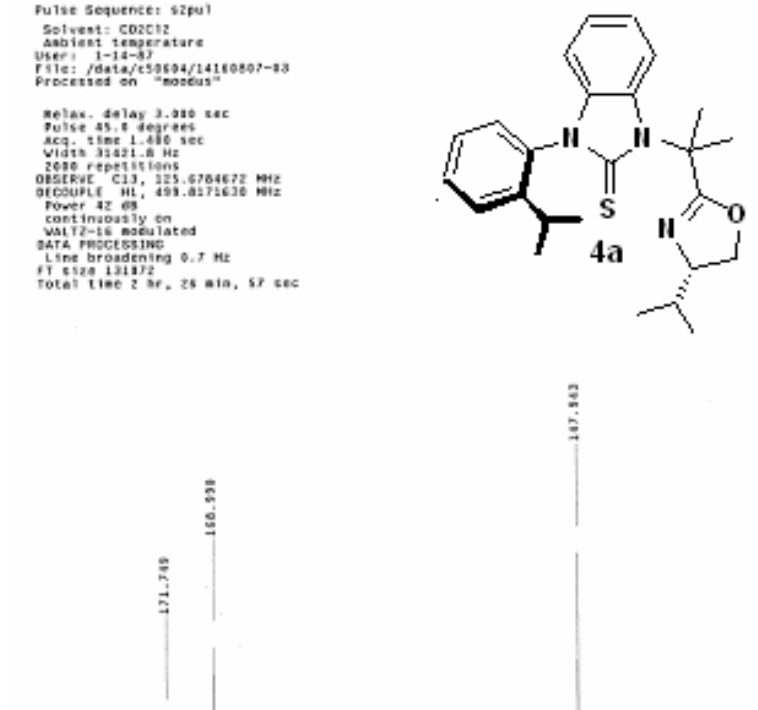

ํํํ

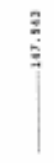

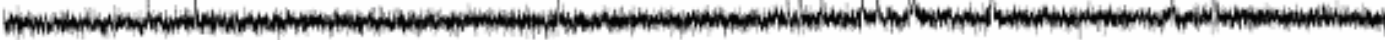

170

160

150

140

130

120

1

눌

药

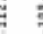

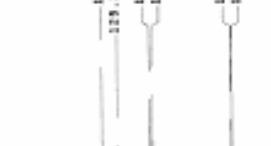


nex?

huise sequace: stpul

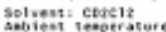

Ther 1-14-89

melax gelay san sey

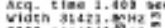

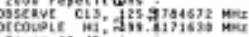

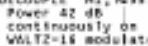

brostention o. $\mathrm{He}$

Tondite istort?

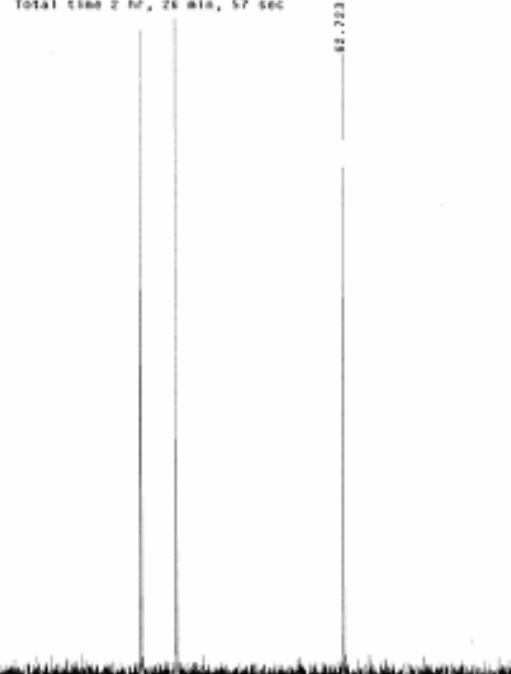

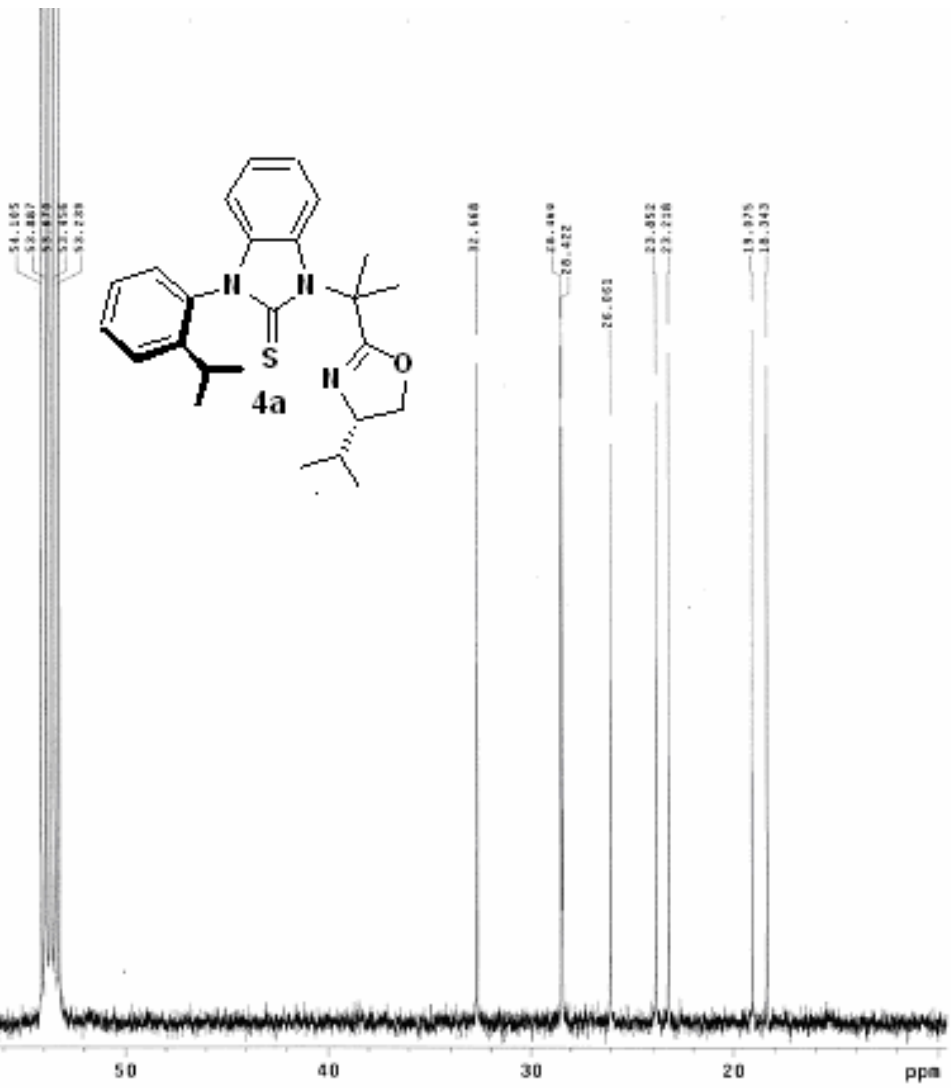



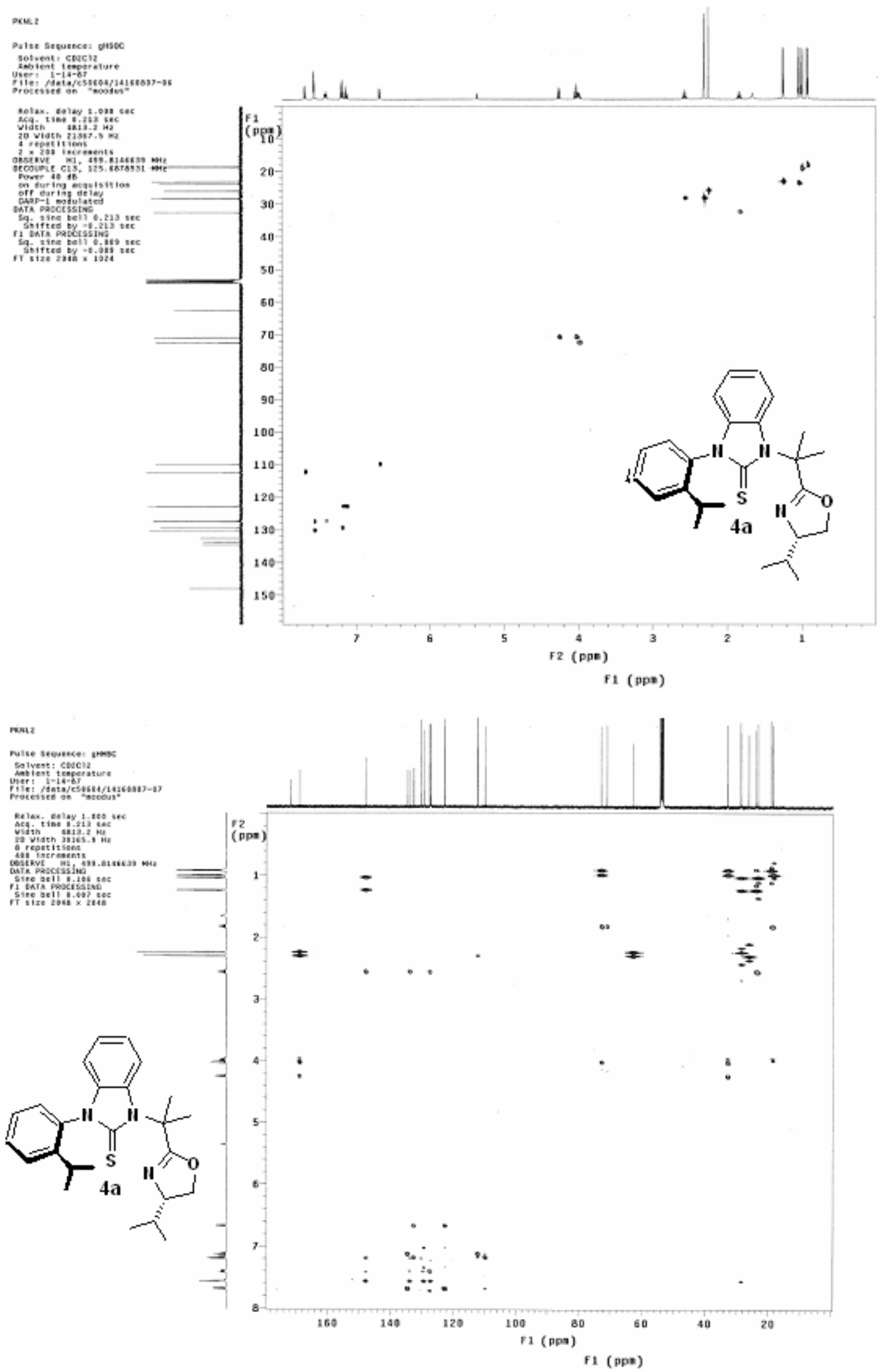
9012

pvise Sequente: gavors

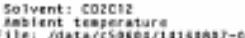
Drocesset on mostus"

Eilax: felog seon set

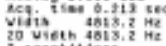

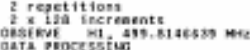

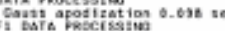

couts ofotectition 0.024 sec

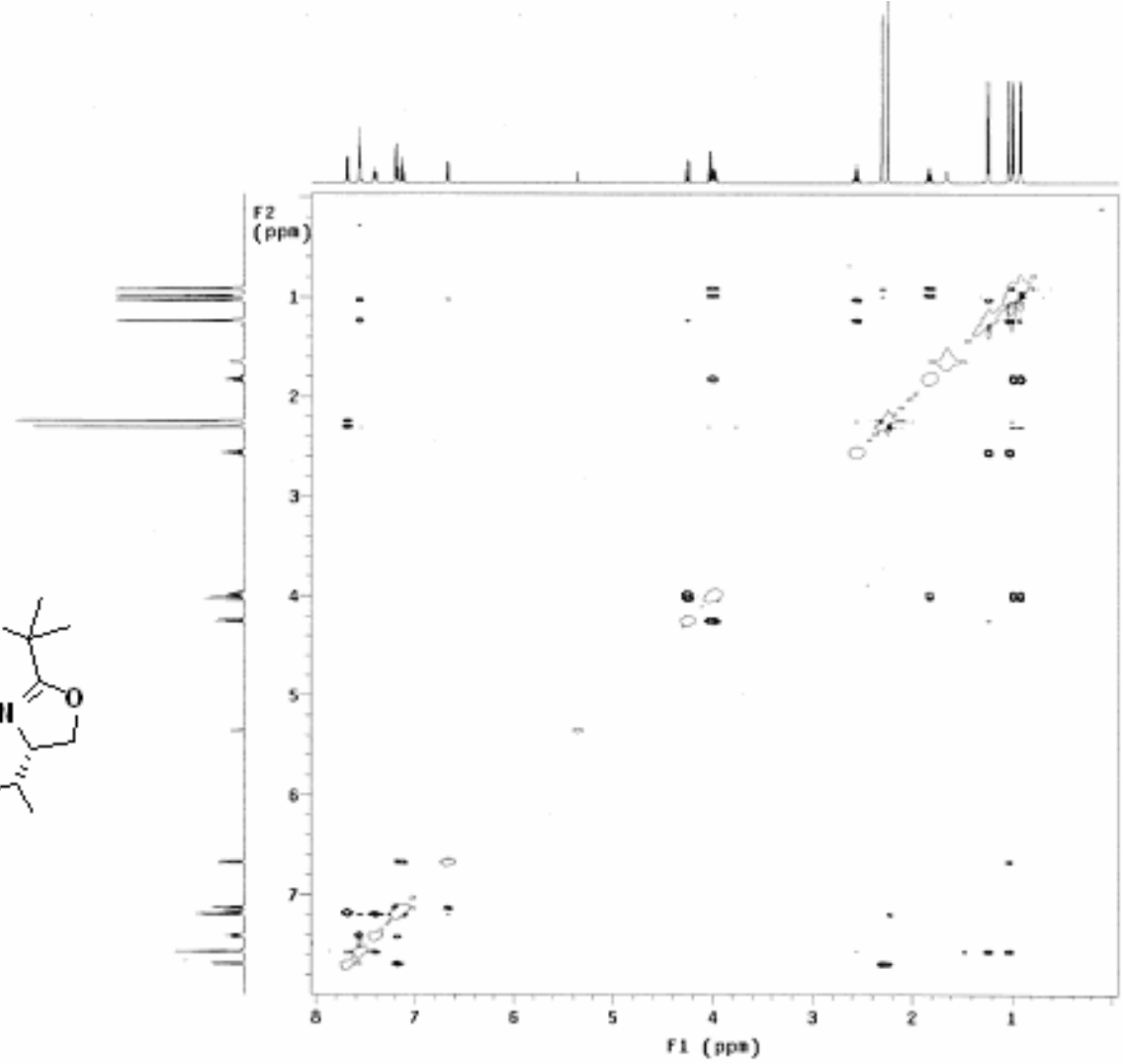

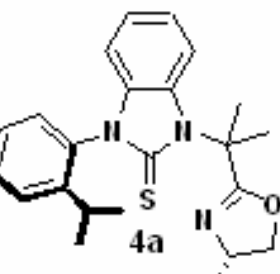

$-$ 
พอนเะ

Dulse seavencer poos

Dolven: cozelz

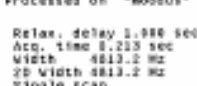

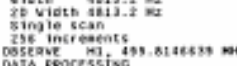

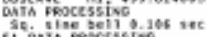

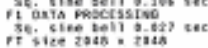
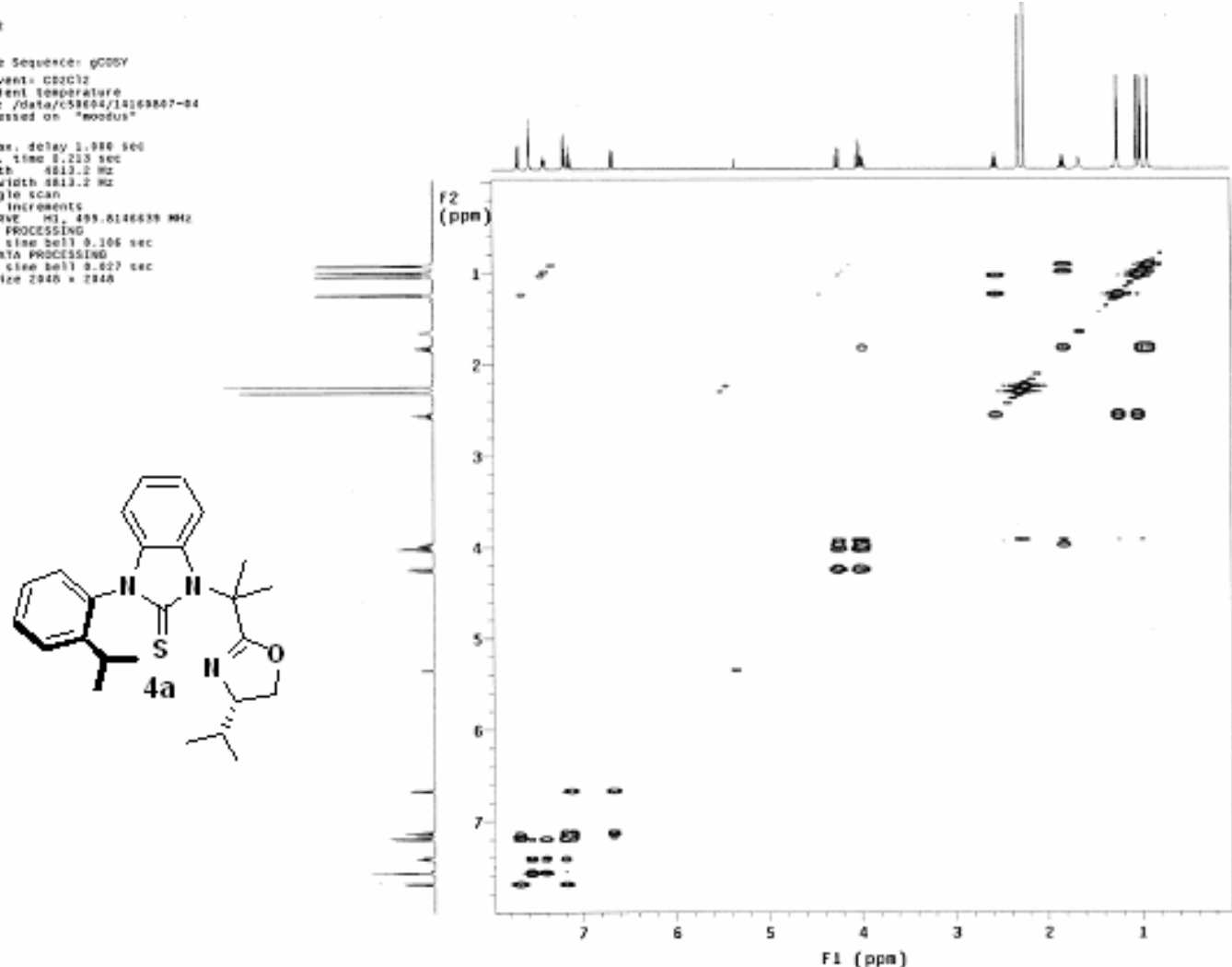

1 (ppa)

PINL_21 1 13-63 RT:023-0.84 AN: 41 NL: 6.88 E7 T: FTIS + P ESIFI IIms [100.00-600.0g

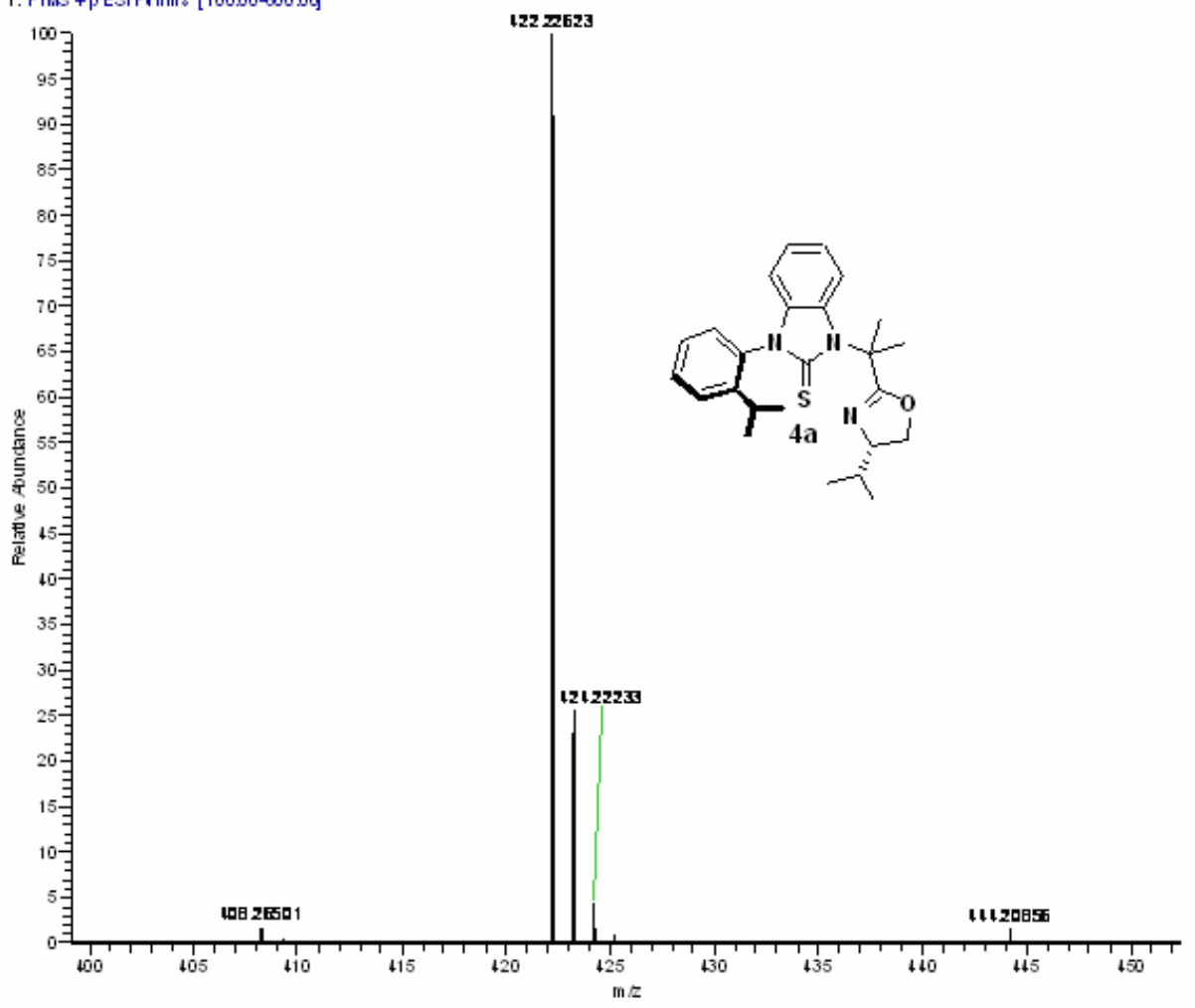




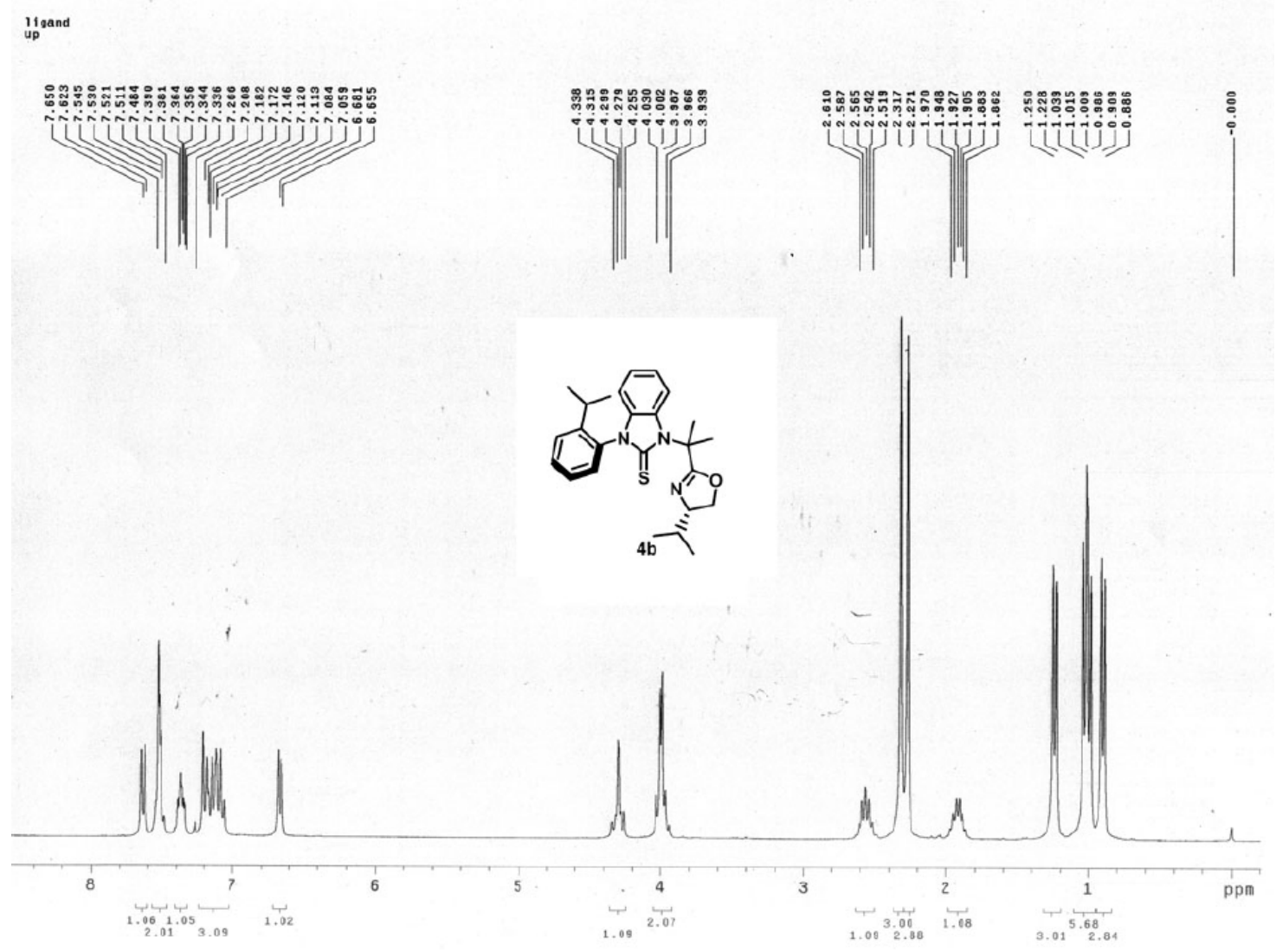


C1s-ipr ipr ilgand up
$300 \mathrm{C}$

Pulse Sequence: save

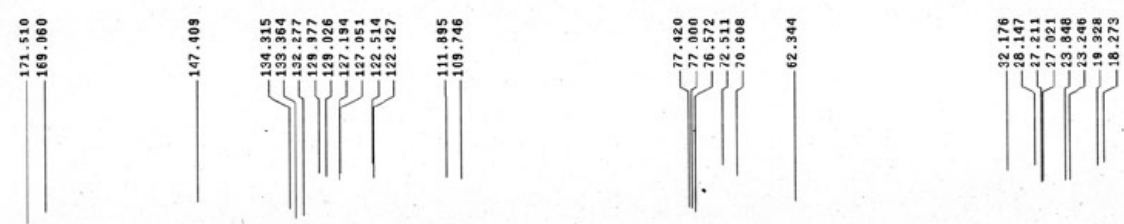

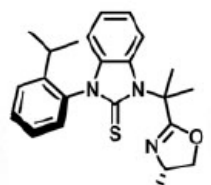

4b

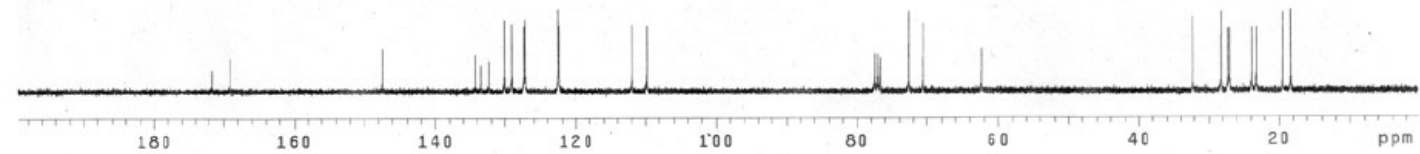

P 1 NL_ $22=17-46$ RT: $0.30-0.74$ AN: 30 NL: $5.32 E 7$

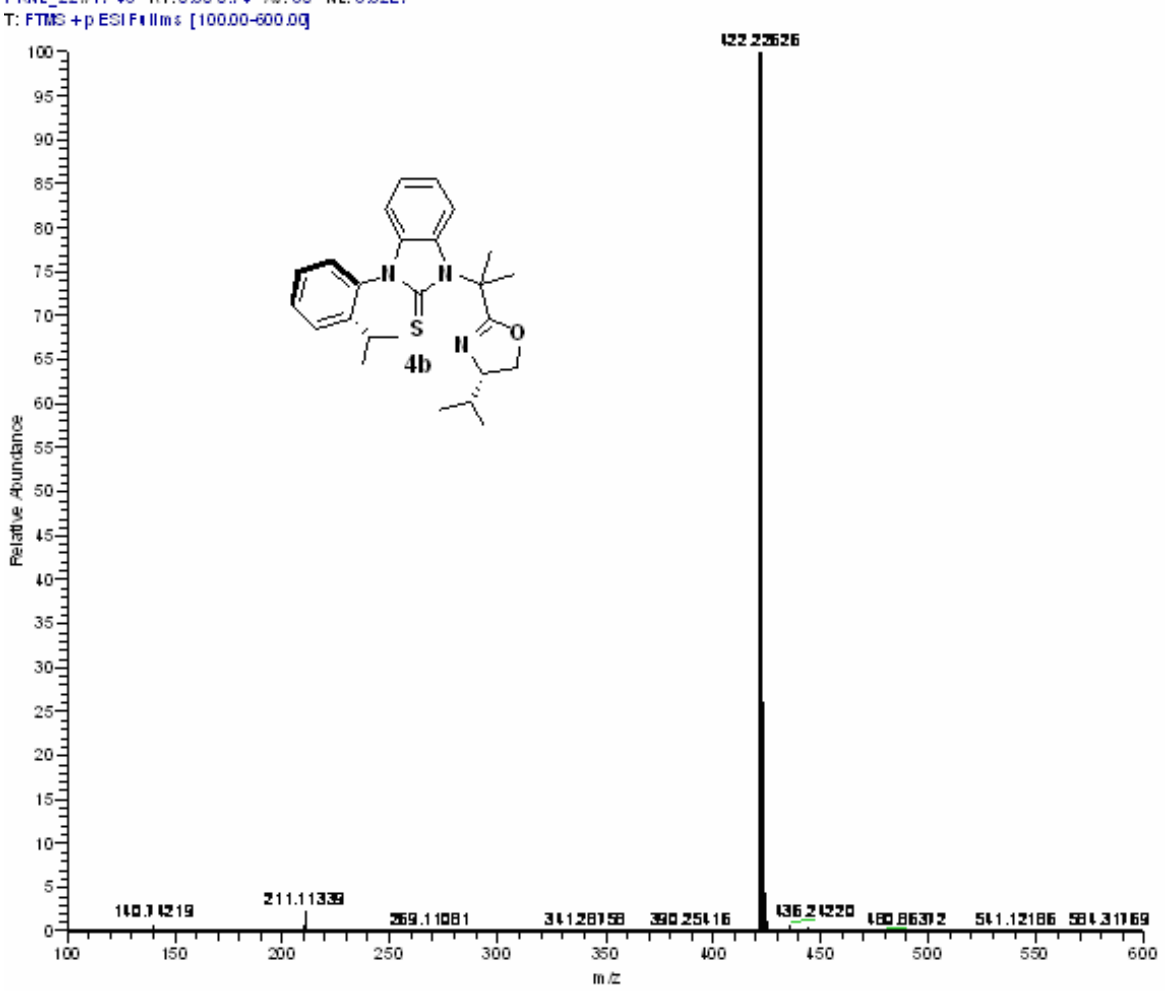



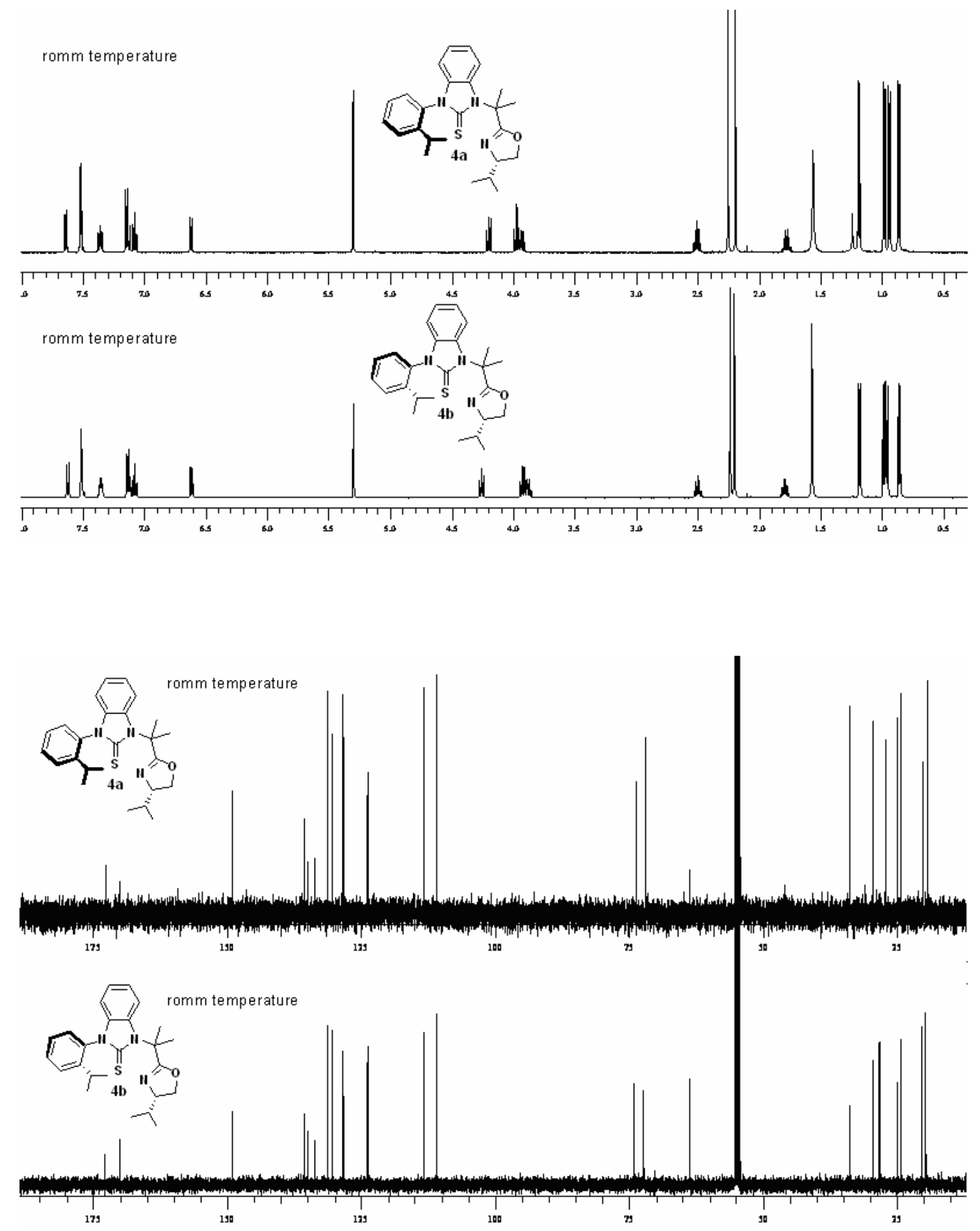


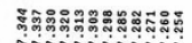

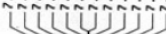

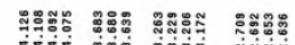

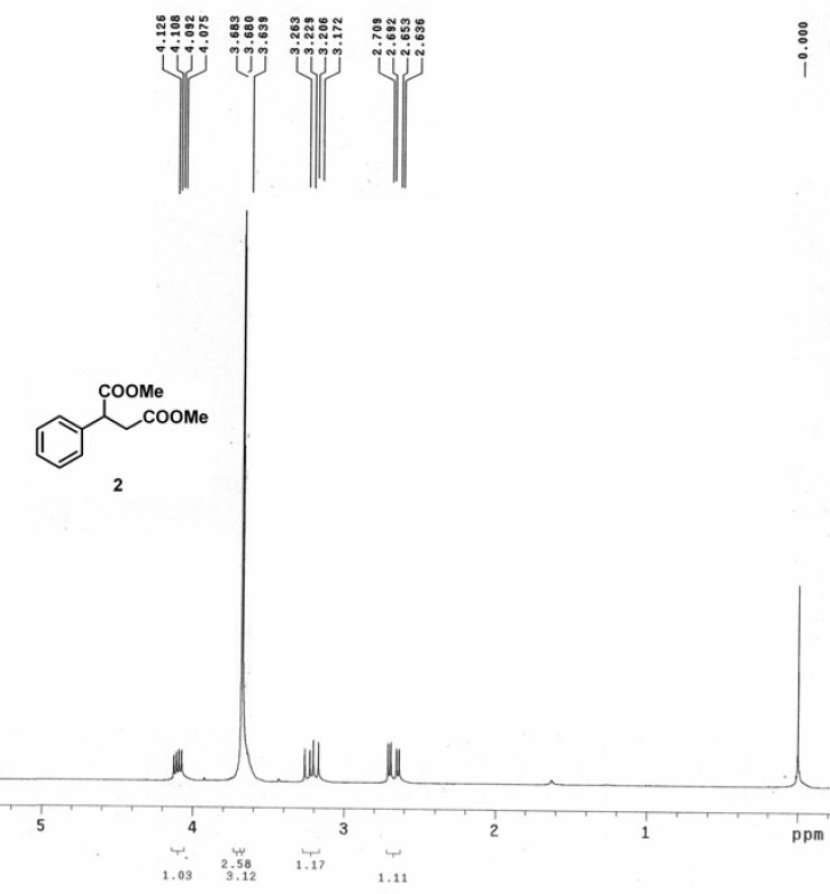

๖ั

N

11angogo
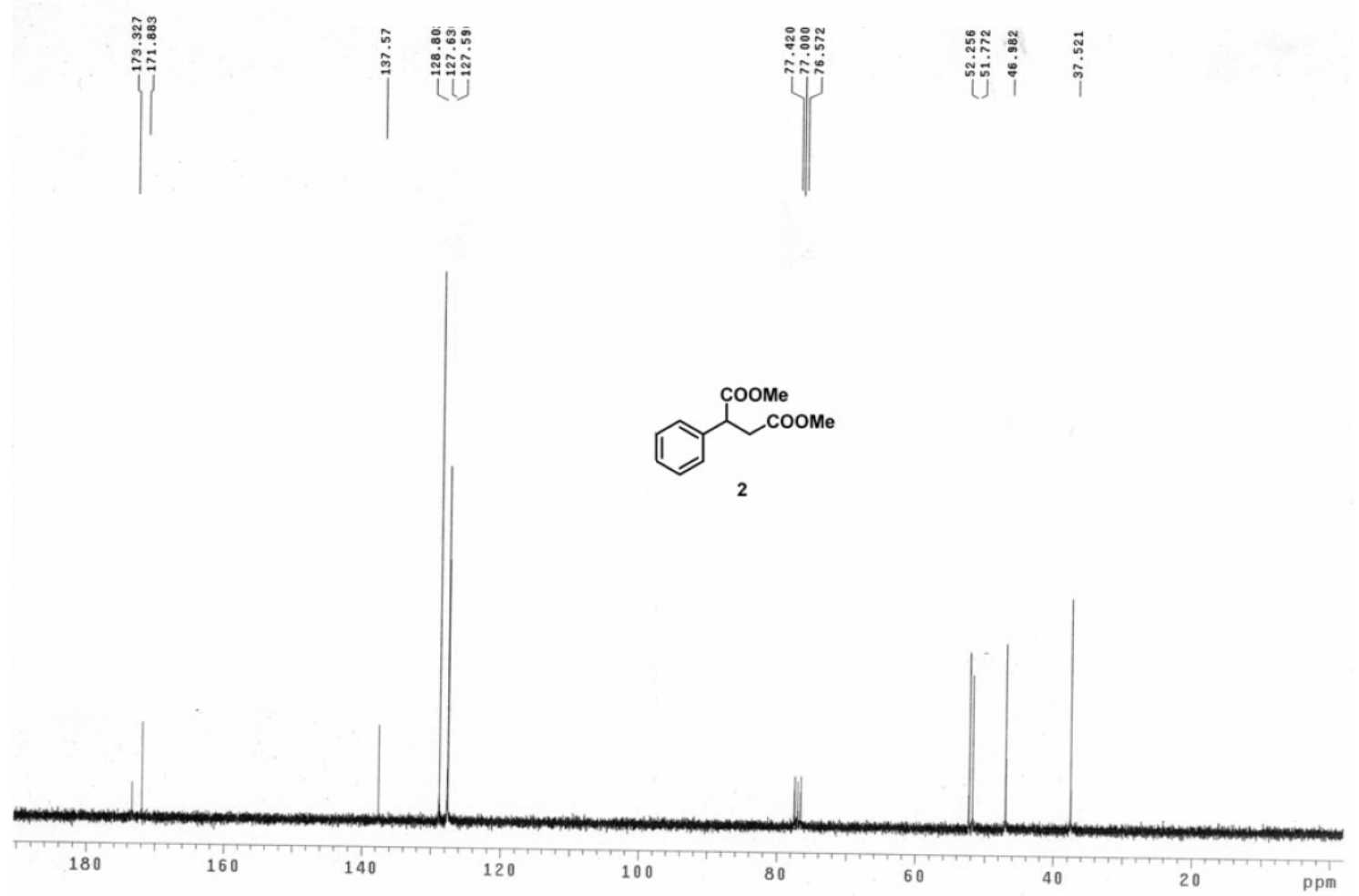


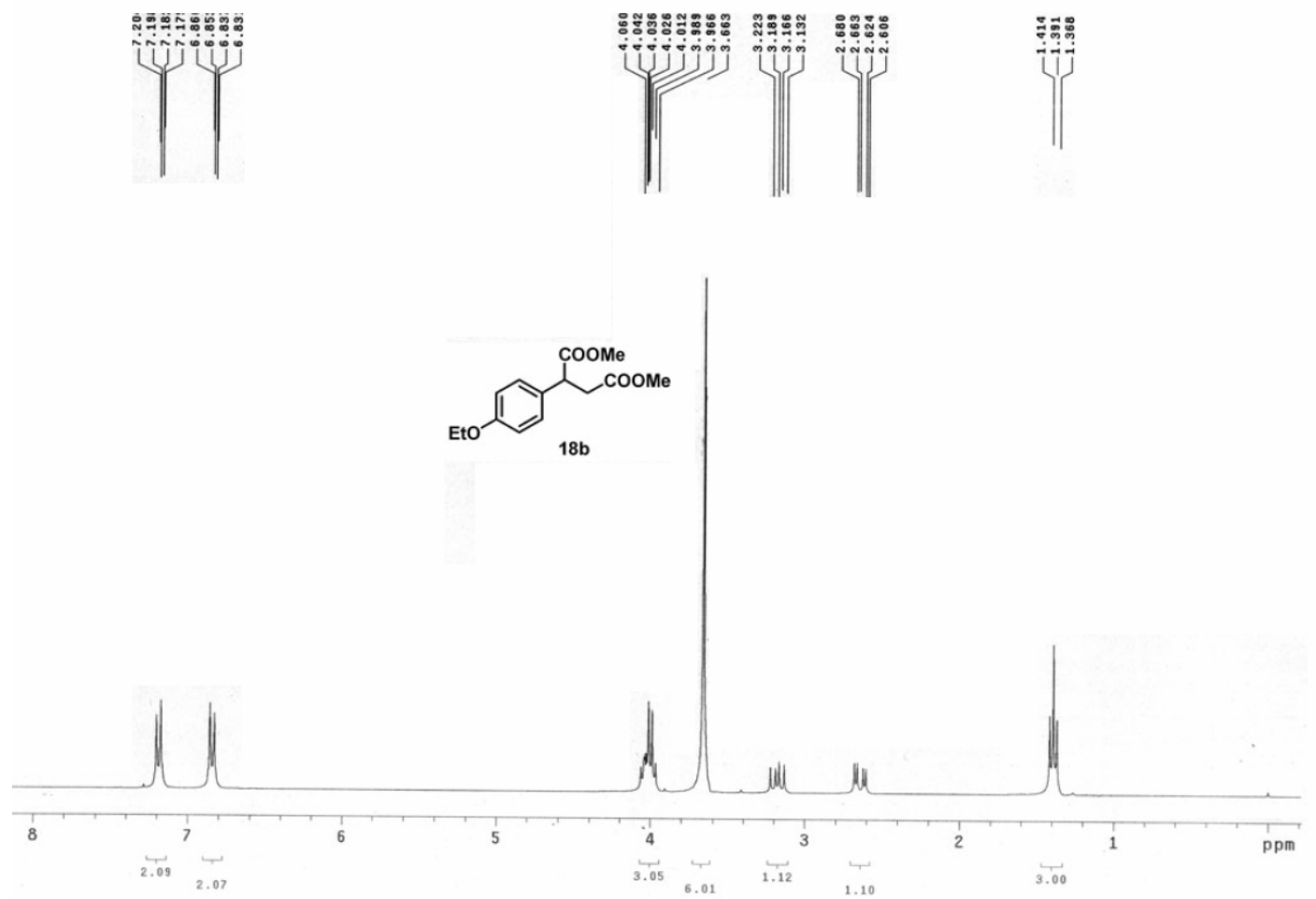

p-ethoxystyrene

Pulse Sequence: s2pul
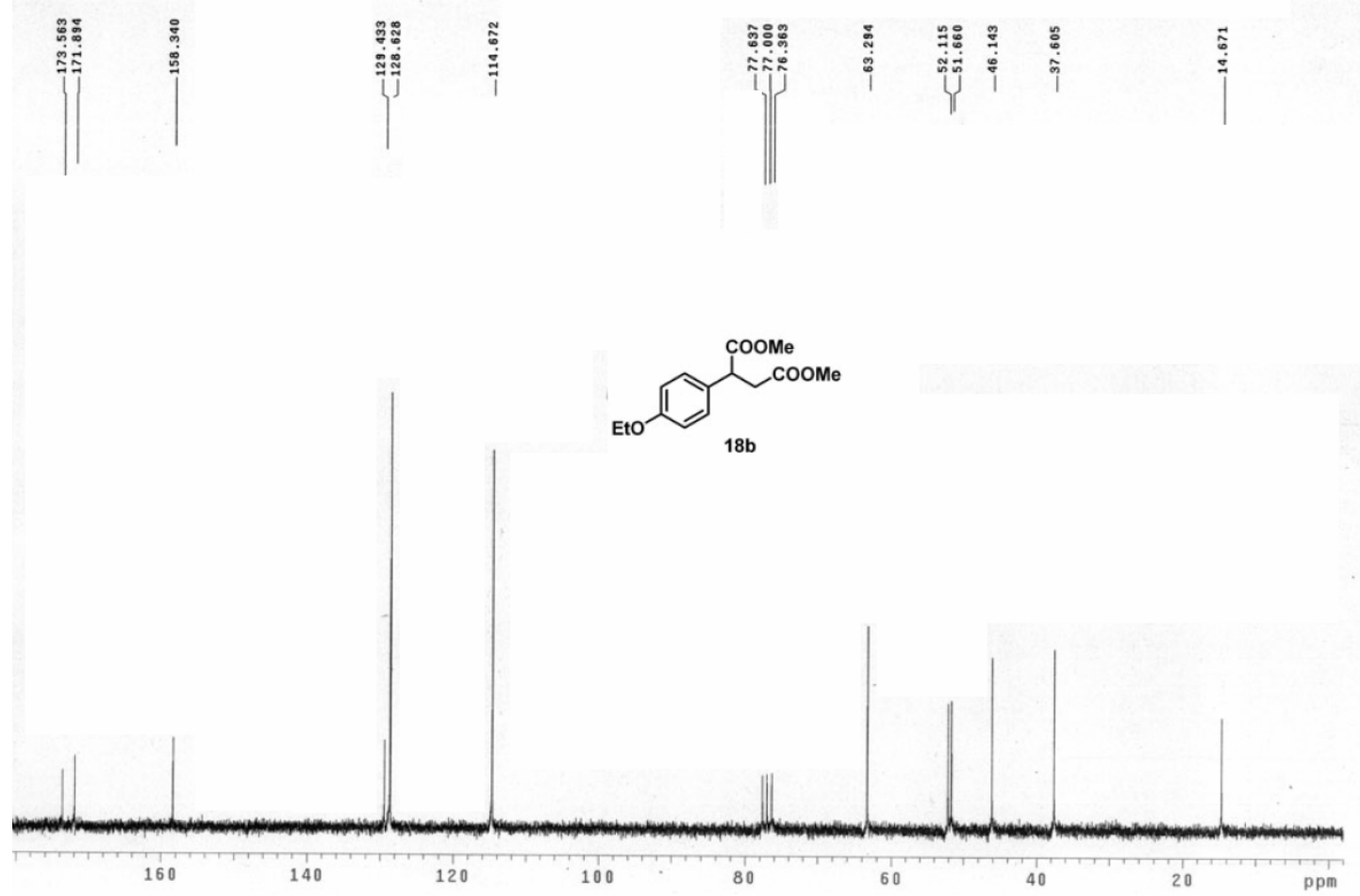


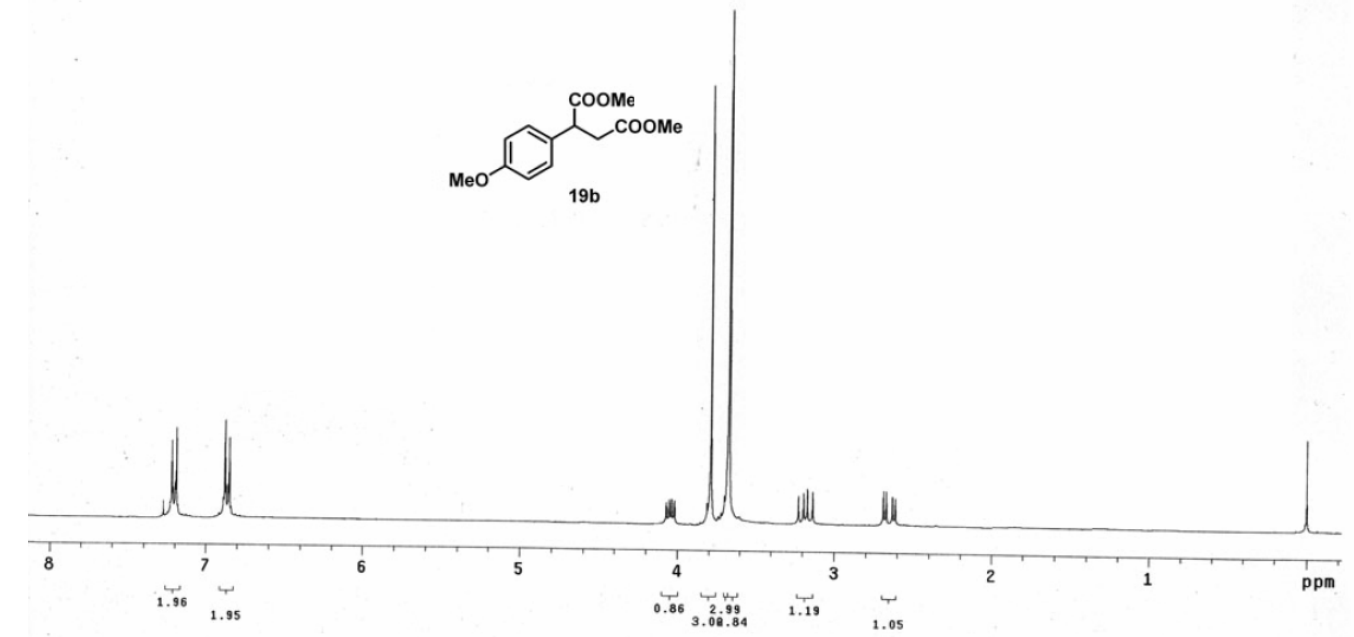

p-methoxystyrene

|

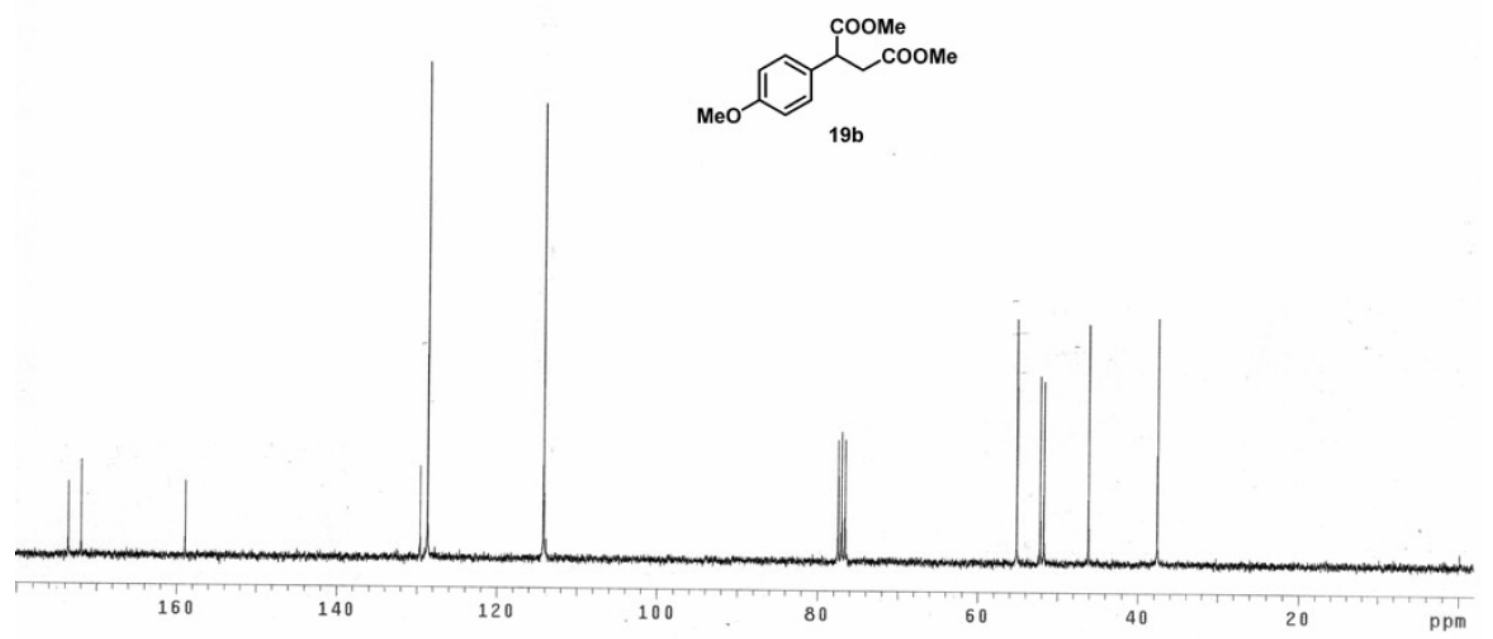




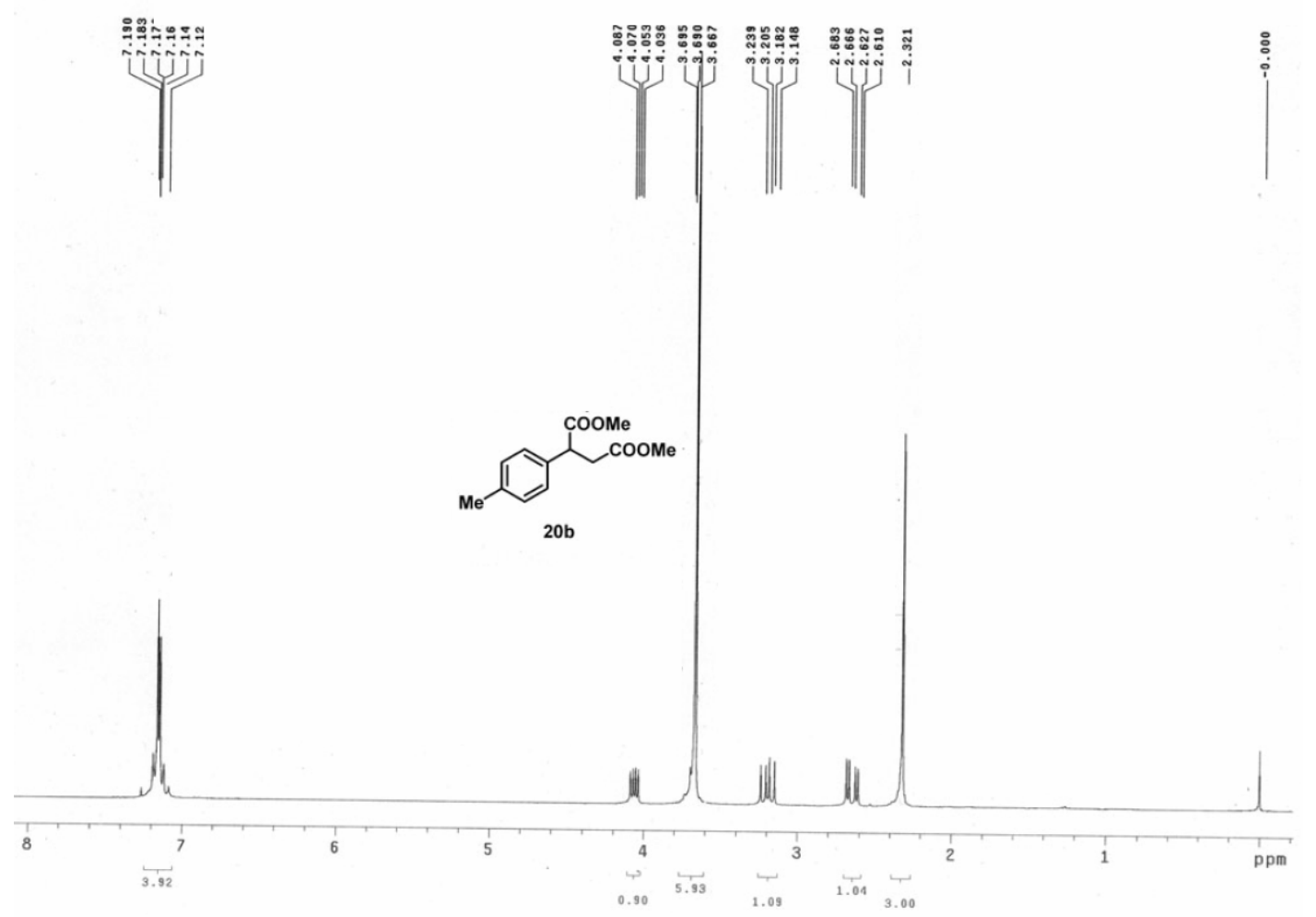

p-aethyistyrane
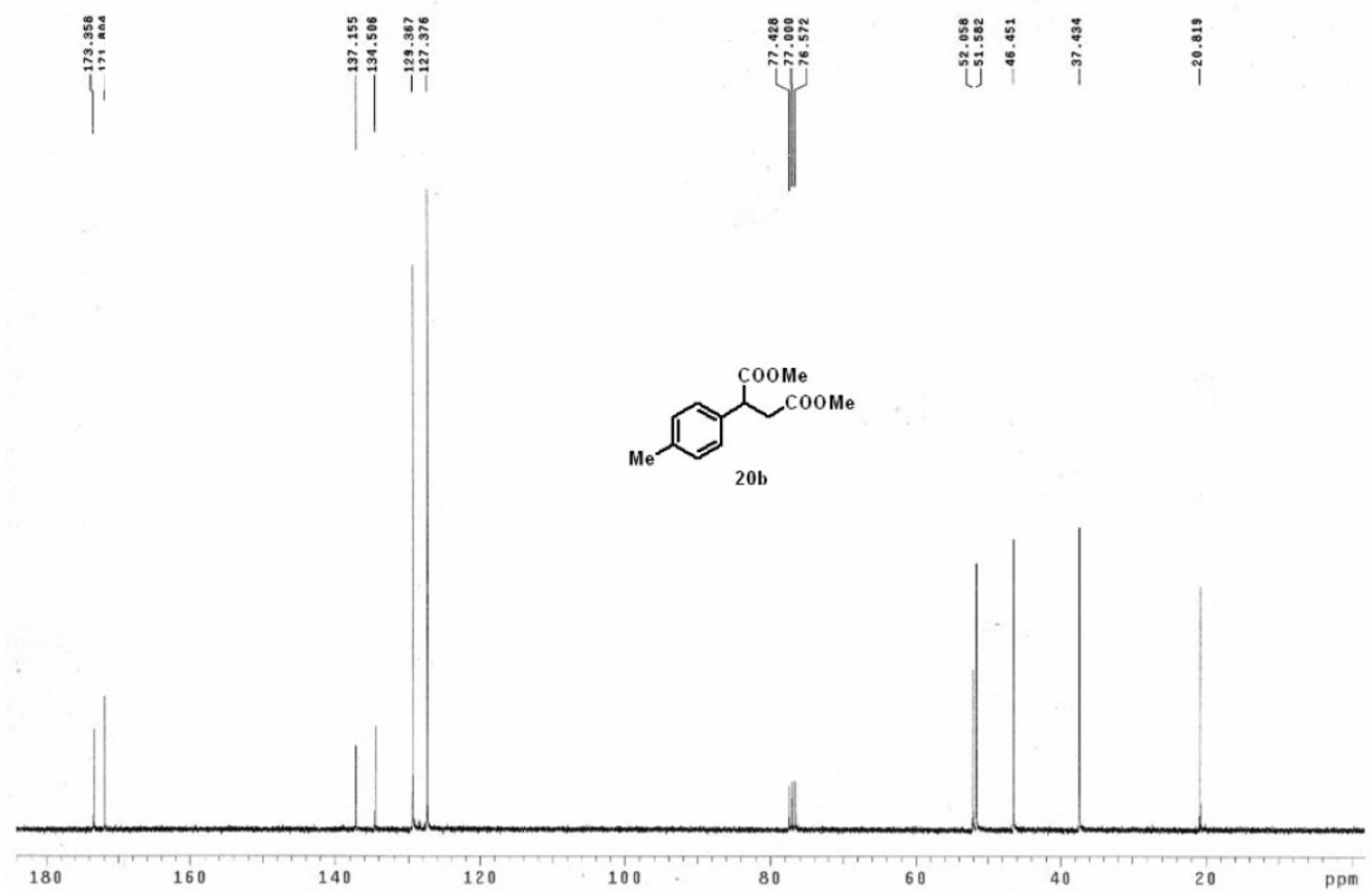
Chloro up 020

Archive directory: /export/home/vner 1/vnmrsys/date
Sample directory:

Pulse Sequence: s2pur

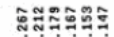

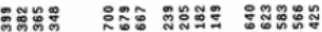

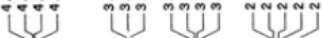

iñĩ
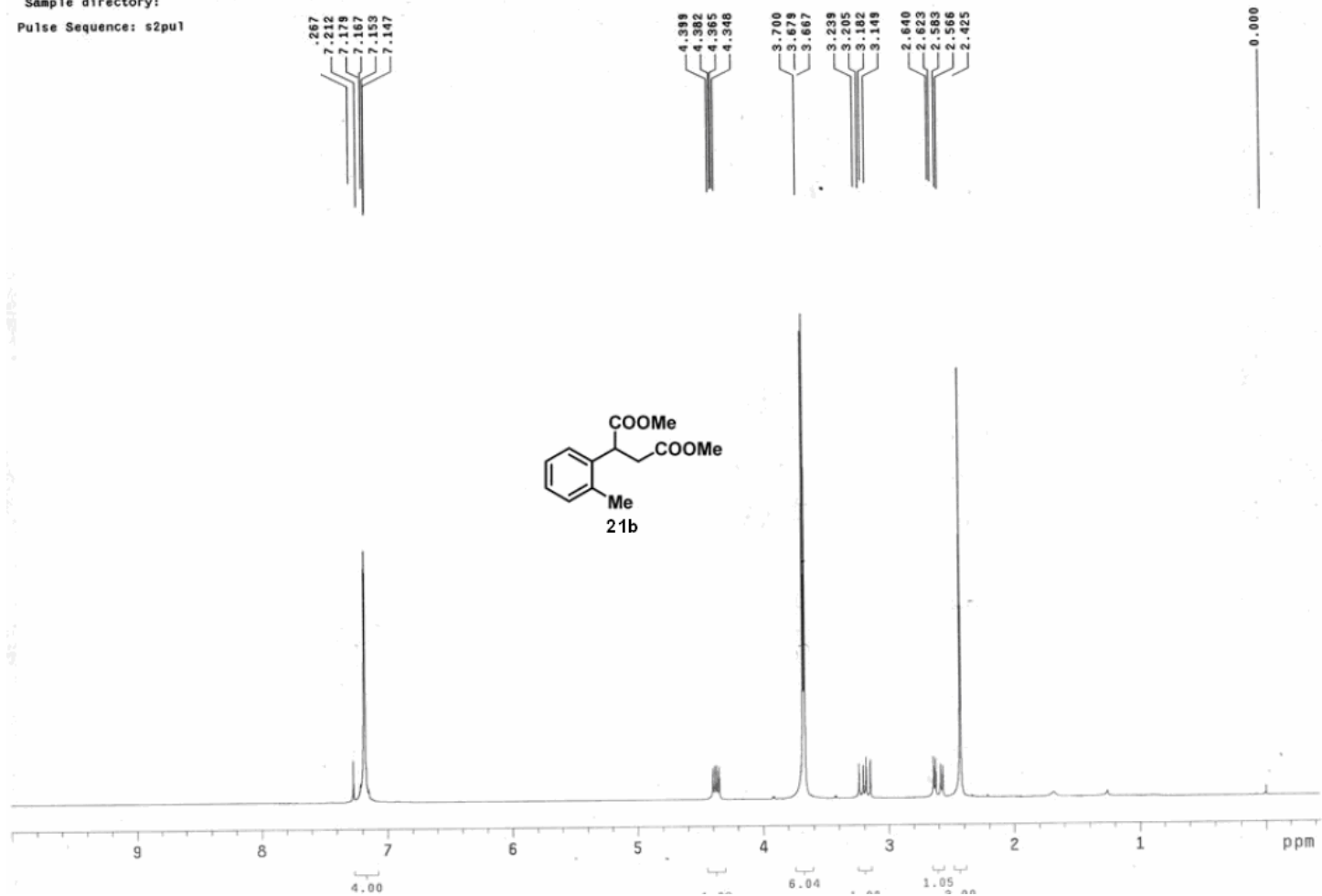

${ }_{300}^{\text {C13- o-Me dimethoxylation product }}$

Pulse Sequence: s2pu1
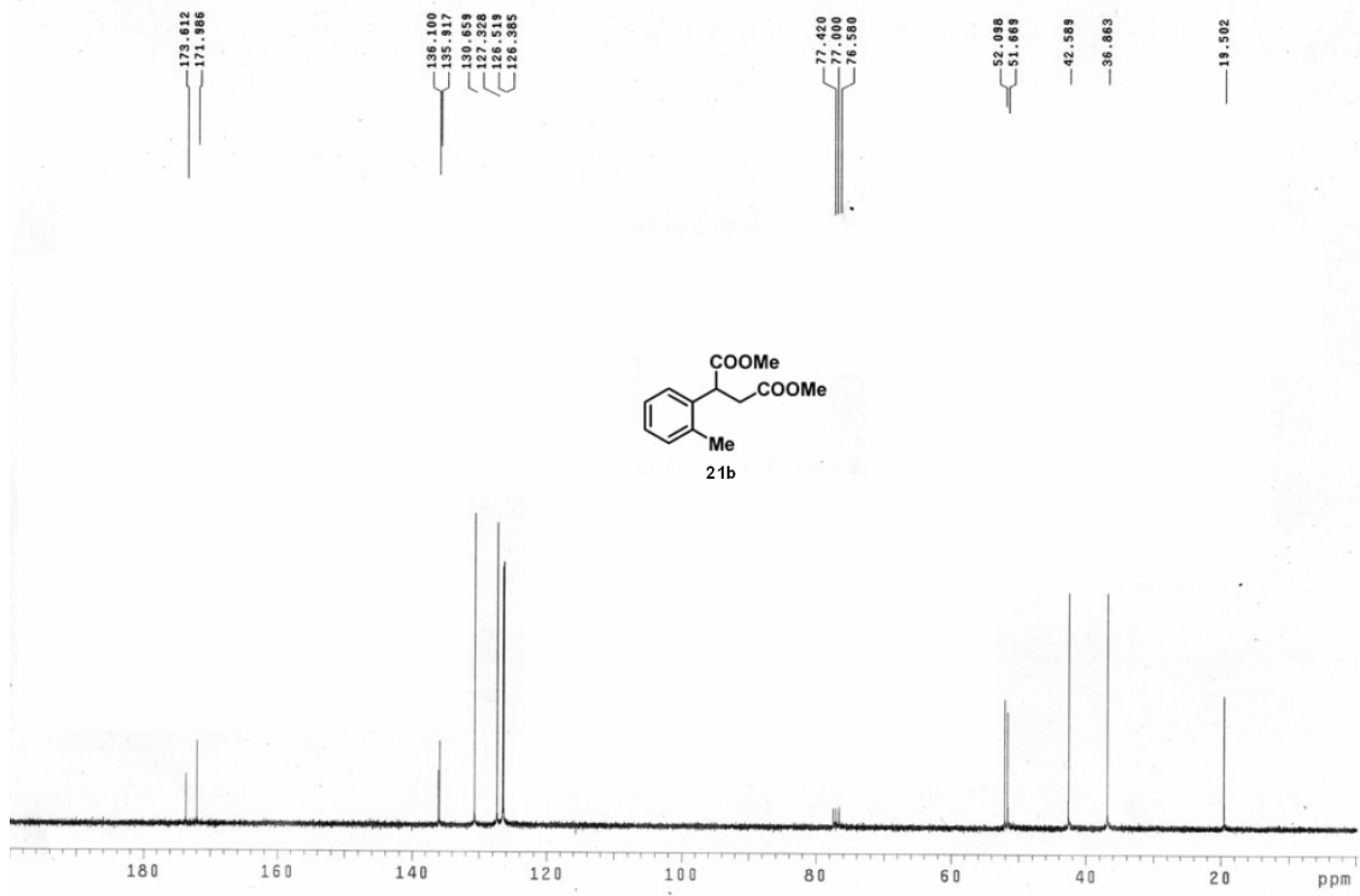


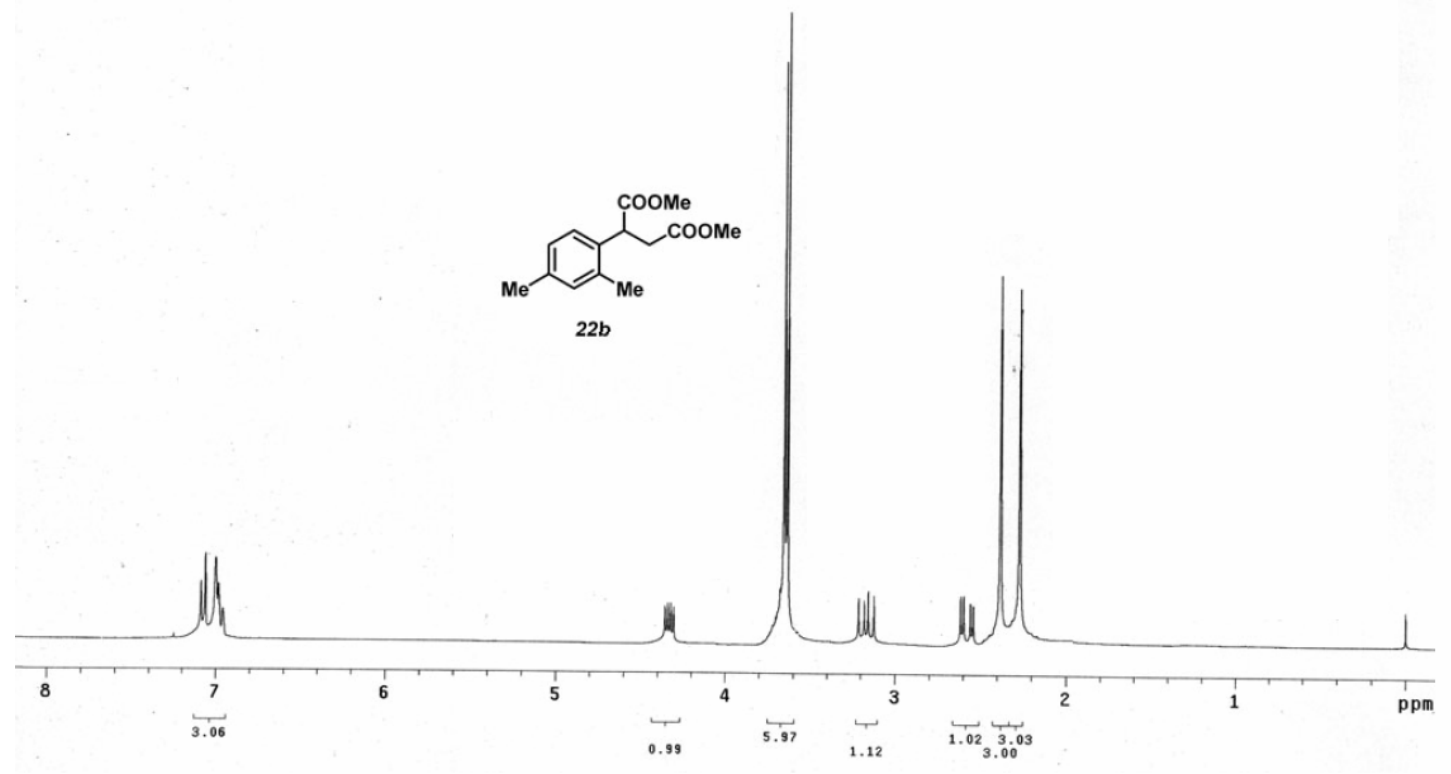

2,4-dimethyistyrene
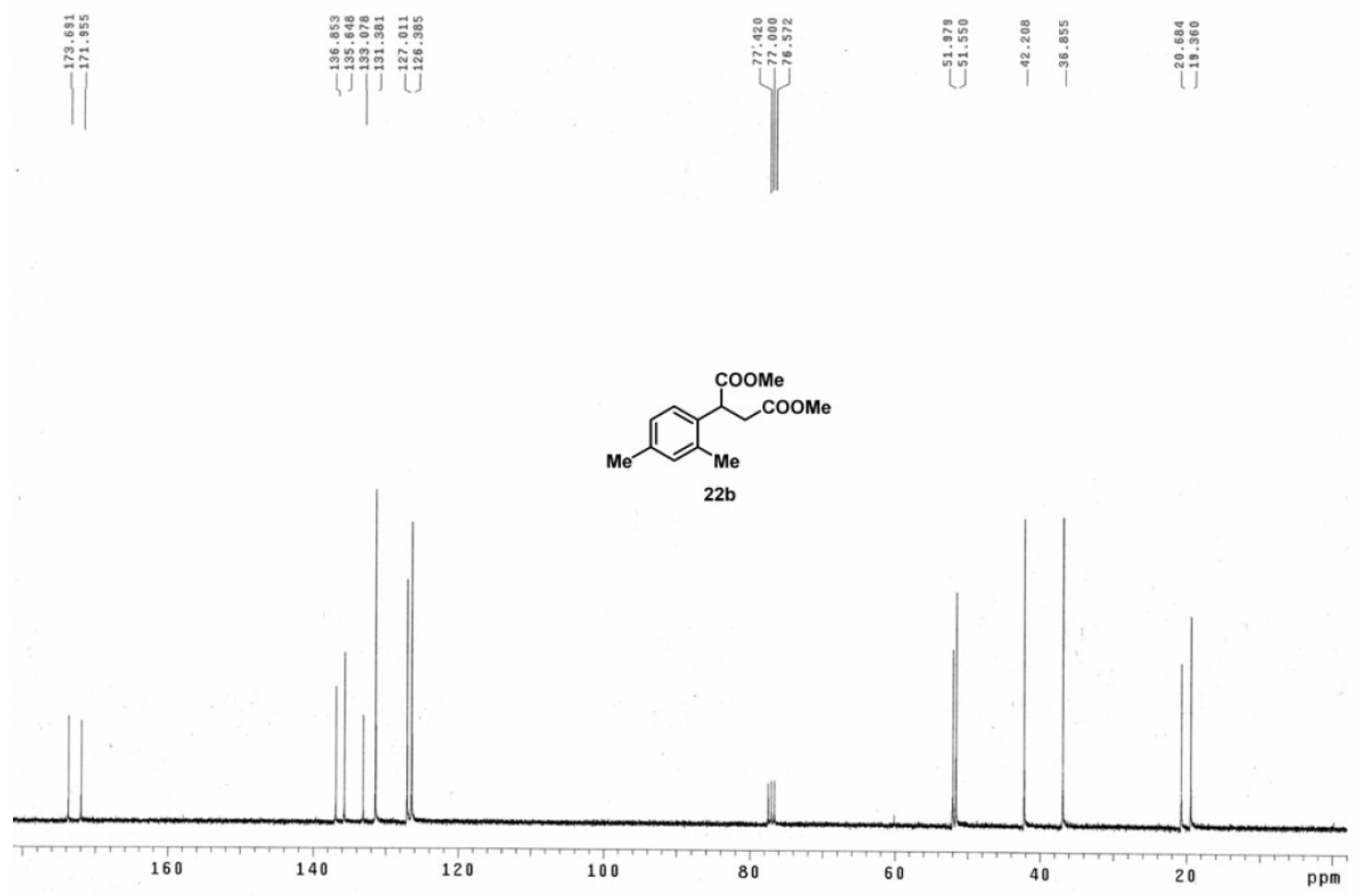


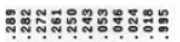

ก กิก

ว

|

نิ

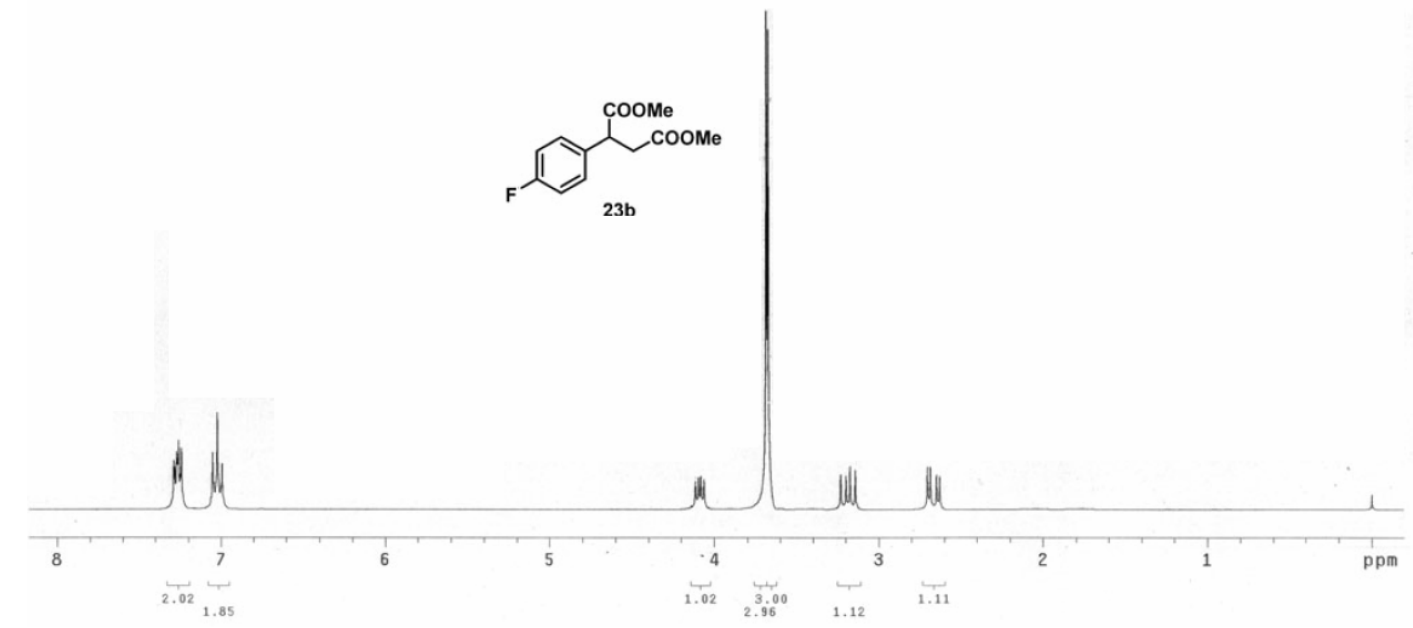

-fluorostyrene

Pulse Sequence: s2pur
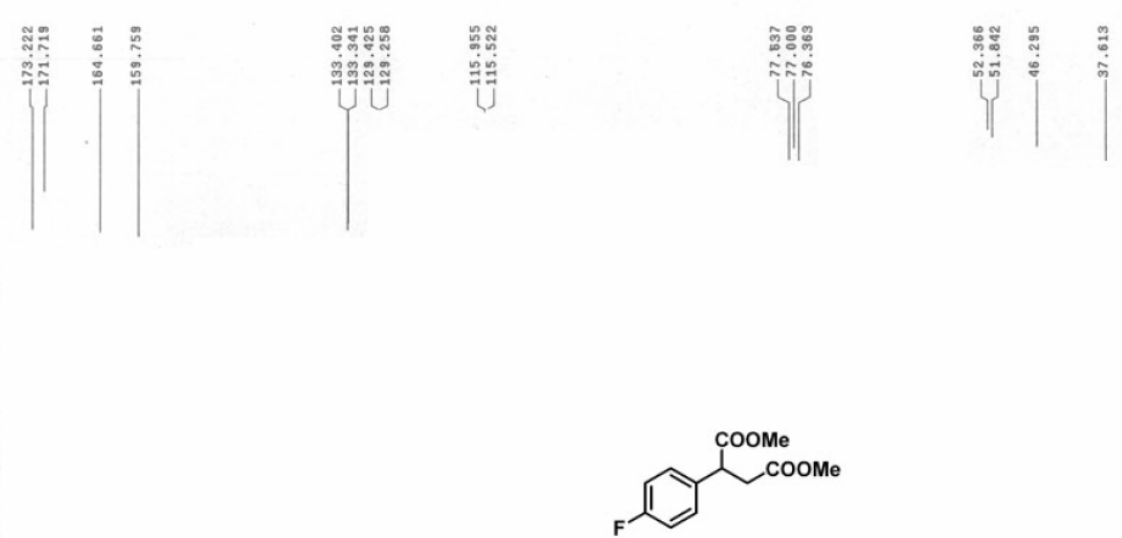

$23 b$

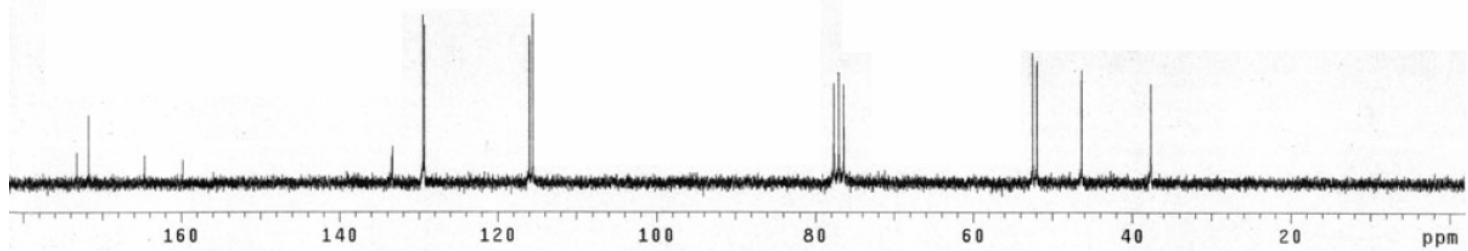


$\underset{2006-3-21}{11 a n g b o}$

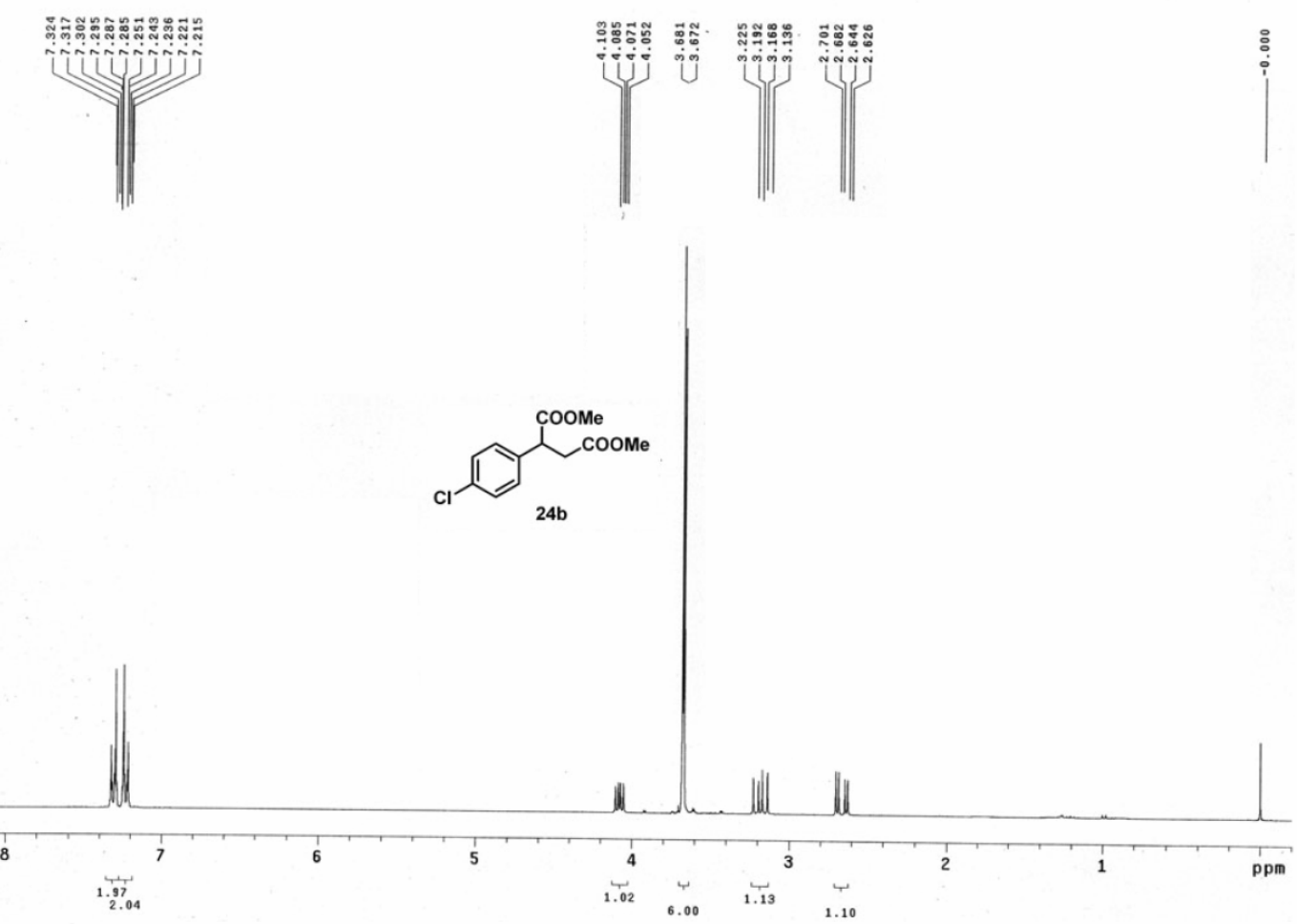

11angobo
$2006-3-21$
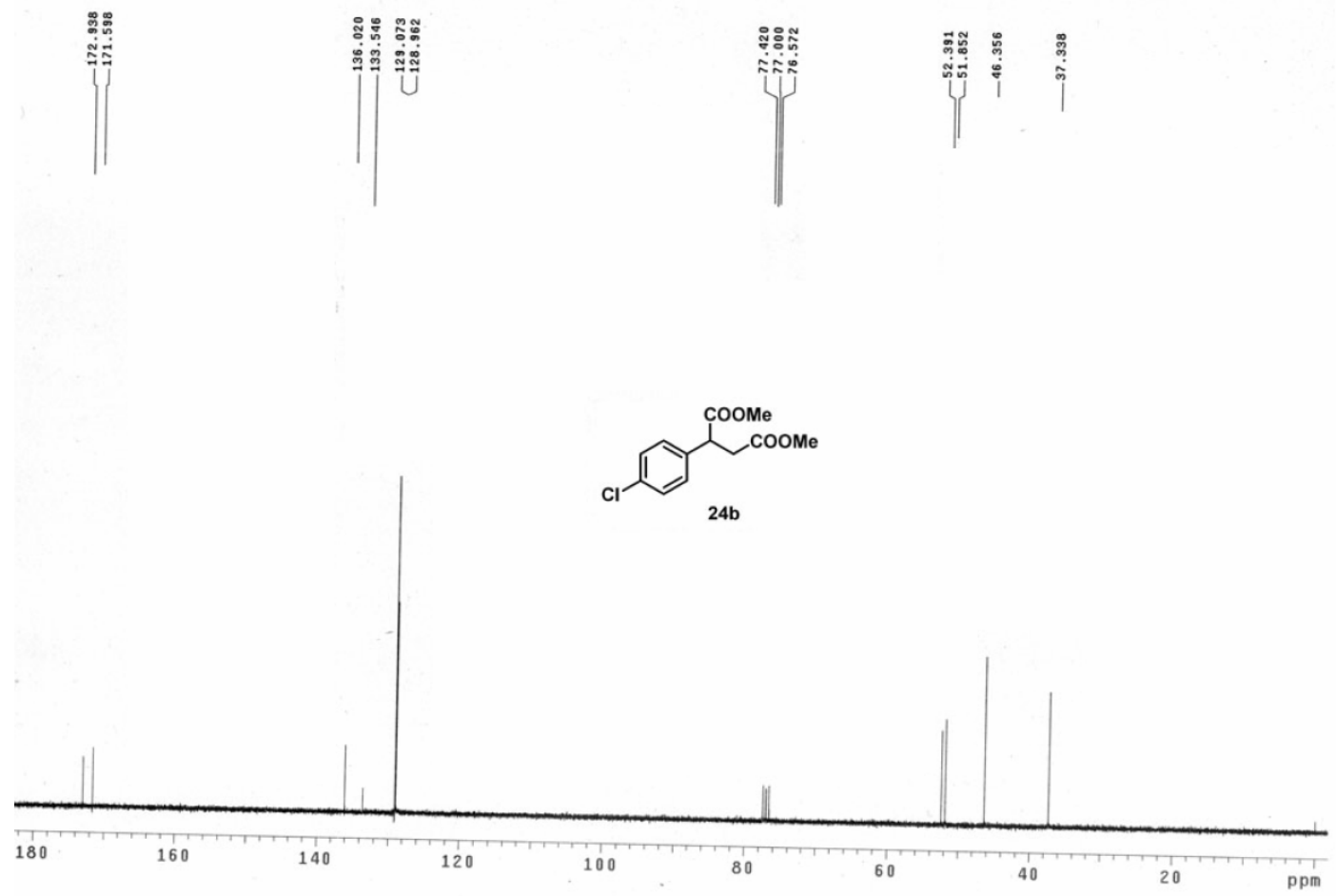


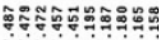

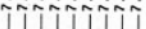

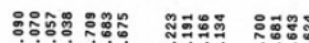

ఏั

coOMe

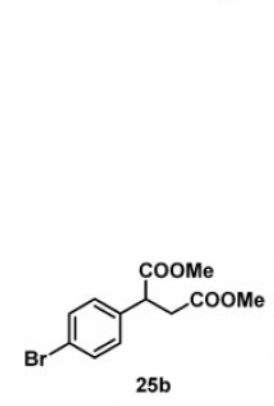

25b

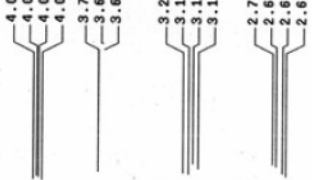

$$
\text { !े }
$$
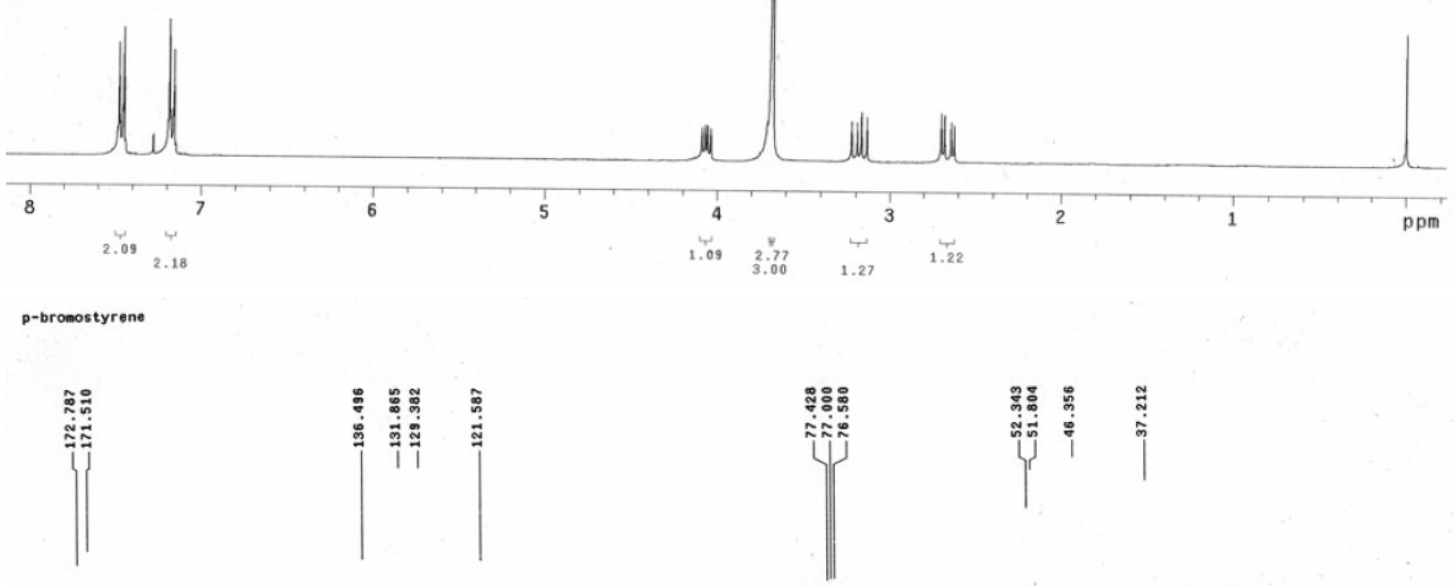


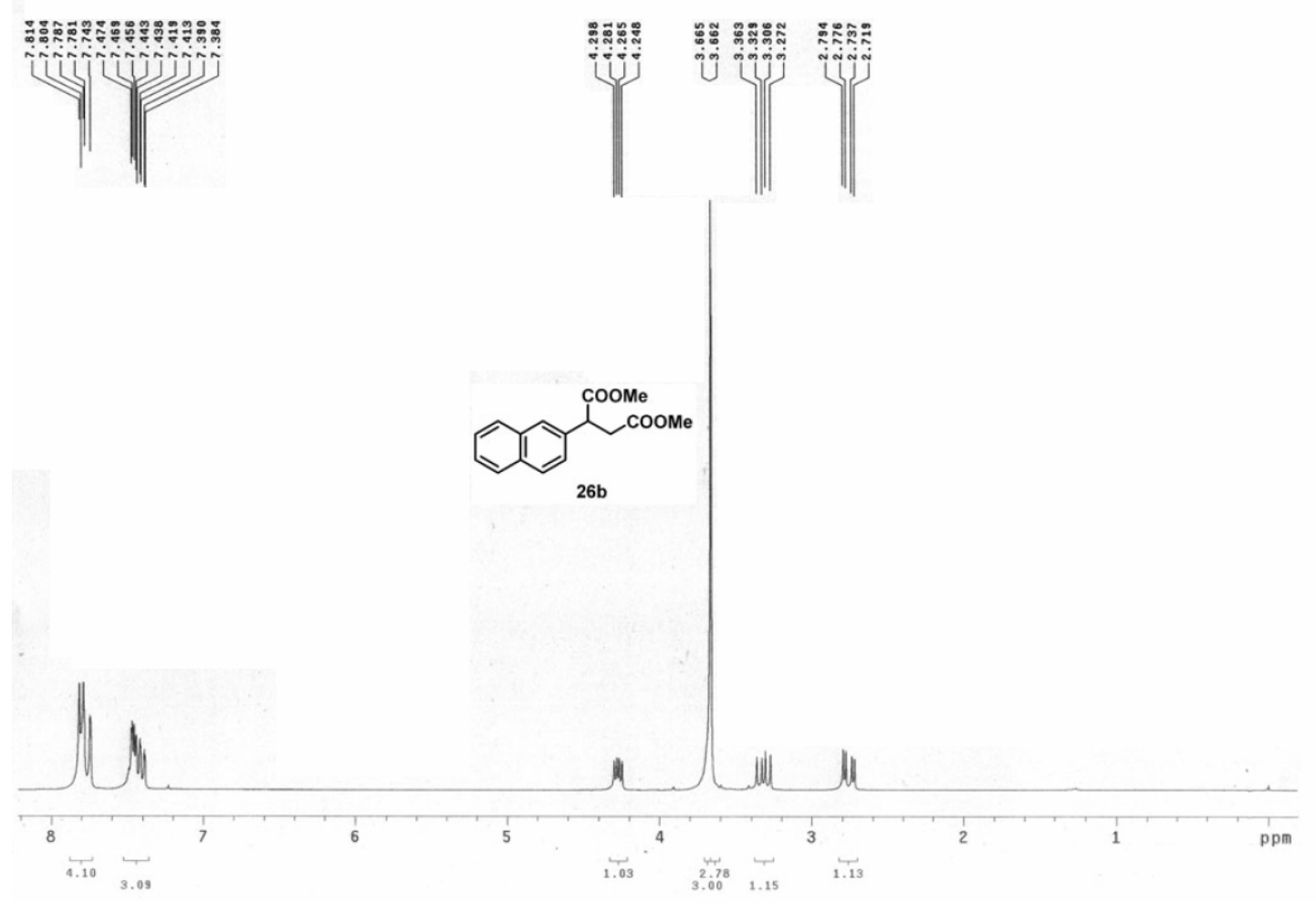

vinylnaph

Pulse Sequence: s2pul
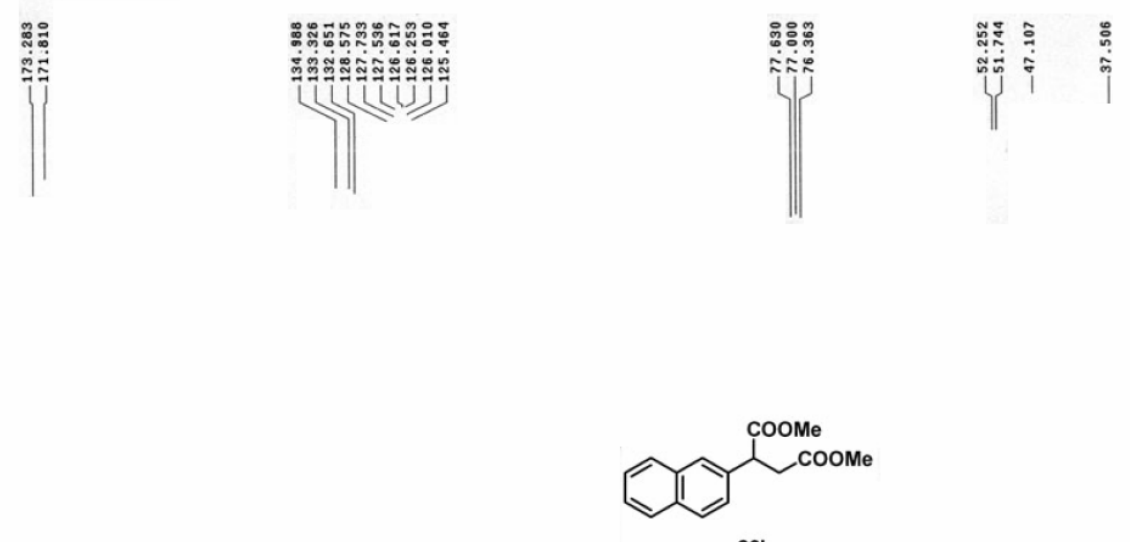

26b

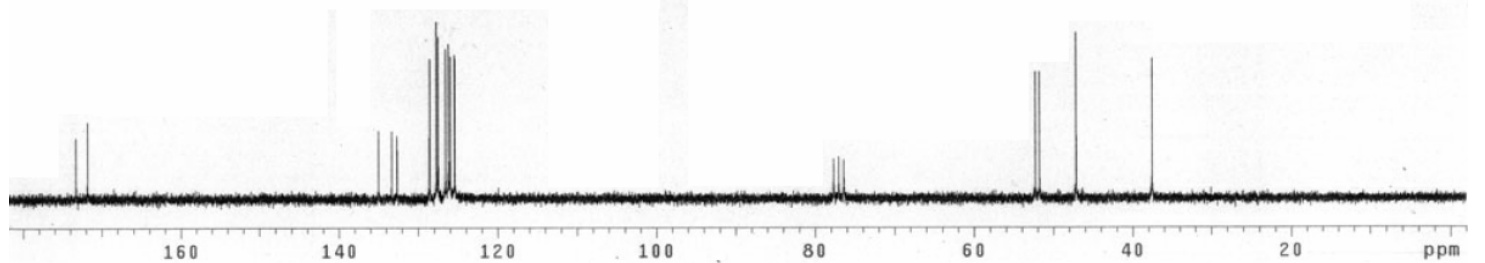




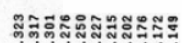

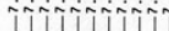

나

1.

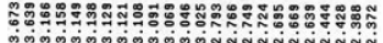

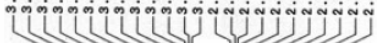

| | | | |

Coome

27b

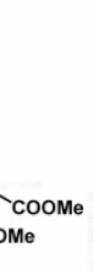

네네

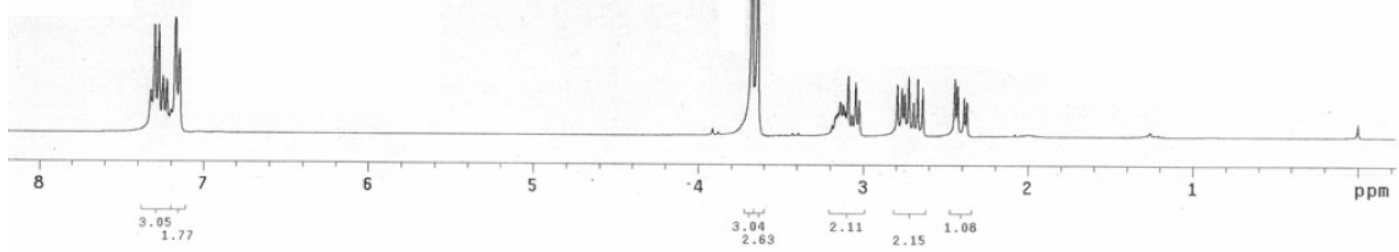

vinylbenzene

Pulse Sequence: s2pul

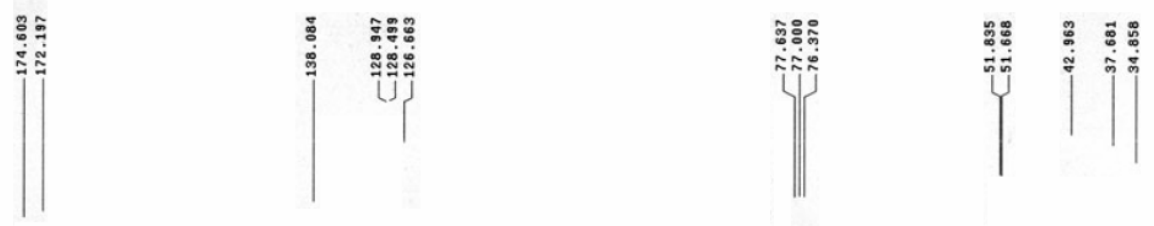

27b

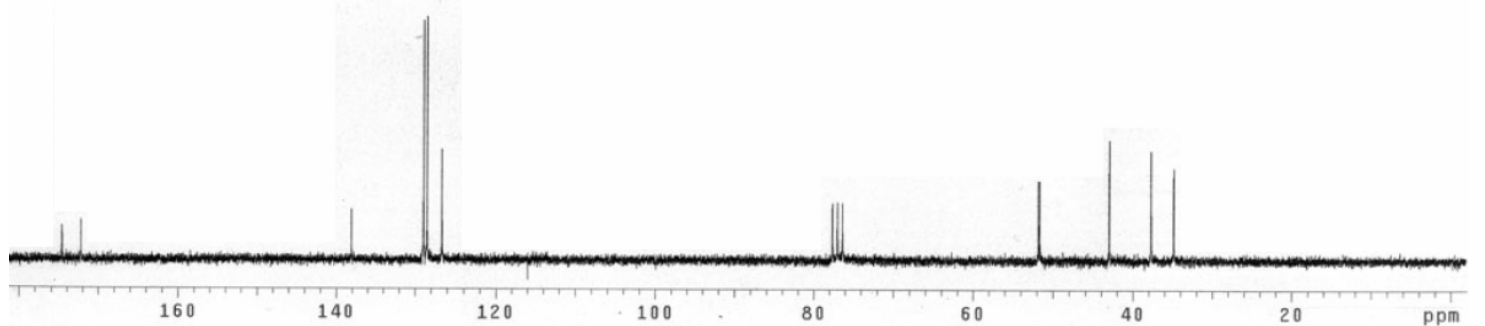


Full authorship of reference 20:

Gaussian 03, Revision C.02. Frisch, M. J.; Trucks, G. W.; Schlegel, H. B.; Scuseria, G. E.; Robb, M. A.; Cheeseman, J. R.; Montgomery, J. A., Jr.; Vreven, T.; Kudin, K. N.; Burant, J. C.; Millam, J. M.; Iyengar, S. S.; Tomasi, J.; Barone, V.; Mennucci, B.; Cossi, M.; Scalmani, G.; Rega, N.; Petersson, G. A.; Nakatsuji, H.; Hada, M.; Ehara, M.; Toyota, K.; Fukuda, R.; Hasegawa, J.; Ishida, M.; Nakajima, T.; Honda, Y.; Kitao, O.; Nakai, H.; Klene, M.; Li, X.; Knox, J. E.; Hratchian, H. P.; Cross, J. B.; Adamo, C.; Jaramillo, J.; Gomperts, R.; Stratmann, R. E.; Yazyev, O.; Austin, A. J.; Cammi, R.; Pomelli, C.; Ochterski, J. W.; Ayala, P. Y.; Morokuma, K.; Voth, G. A.; Salvador, P.; Dannenberg, J. J.; Zakrzewski, V. G.; Dapprich, S.; Daniels, A. D.; Strain, M. C.; Farkas, O.; Malick, D. K.; Rabuck, A. D.; Raghavachari, K.; Foresman, J. B.; Ortiz, J. V.; Cui, Q.; Baboul, A. G.; Clifford, S.; Cioslowski, J.; Stefanov, B. B.; Liu, G.; Liashenko, A.; Piskorz, P.; Komaromi, I.; Martin, R. L.; Fox, D. J.; Keith, T.; Al-Laham, M. A.; Peng, C. Y.; Nanayakkara, A.; Challacombe, M.; Gill, P. M. W.; Johnson, B.; Chen, W.; Wong, M. W.; Gonzalez, C.; Pople, J. A. Gaussian, Inc., Wallingford CT, 2004. 